Teoria de estratificação e condições de regularidade

Vanessa Munhoz Reina Bezerra 



\title{
Teoria de estratificação e condições de regularidade
}

\author{
Vanessa Munhoz Reina Bezerra
}

Orientador: Prof. Dr. Raimundo Nonato Araújo dos Santos

Dissertação apresentada ao Instituto de Ciências Matemáticas e de Computação - ICMC-USP, como parte dos requisitos para obtenção do título de Mestre em Ciências - Matemática.

VERSÃO REVISADA APÓS A DEFESA

USP - São Carlos

Agosto/2007 

Aos meus pais, Luiz Eloy Reina Cano e Maria Neusa Munhoz Reina que me ensinaram a admirá-los.

Ao Wesley de Souza Bezerra por seu amor e sua paciência. 



\section{Agradecimentos}

Agradeço a Deus por mais uma conquista.

Ao meu orientador, o professor Raimundo pela excelente orientação.

Ao professor Marcelo Saia por ter acreditado em mim, e me proporcionado uma chance de mostrar o meu potencial.

A todos meus colegas do ICMC-USP, que tornaram esta caminhada mais agradável, em especial ao Romenique e ao Aurélio por tudo que sofremos ou sorrimos juntos, por nossos estudos, ideais e experiências compartilhadas. Também aos colegas de sala: Amanda pela ajuda com o Beamer, e Márcio pelas dicas e revisão na digitação.

Ao grande amigo Walter, companheiro de toda hora, às vezes filho, outras vezes pai, tantas vezes irmão, mais novo ou mais velho, sempre amigo. Obrigado pelo companheirismo e lealdade. A minha gratidão imensa pela amizade, pela sua infinita paciência, confiança, incentivo e pelos valiosos ensinamentos e conselhos. Também ao Walter e a Ruth pelos bons momentos de descontração proporcionados.

Aos funcionários do ICMC-USP, pela amizade e atenção dispensada.

Aos meus amigos de graduação, que embora estejam distantes, sempre serão lembrados. Em especial a professora Patrícia, cuja amizade e apoio me foram essenciais, sem os quais eu jamais teria coragem, nem estímulo para trilhar este caminho.

Neste sentido, honras e terna gratidão à minha família, que com seu amor e ensinamentos sempre me transmitiram esperança, confiança e a alegria de poder estar sempre de cabeça erguida. Mais que agradecer, eu dedico este trabalho à minha família:

Ao Julian e Luciana que além de serem meus irmãos, são meus grandes e melhores amigos.

Aos meus tios Luiza e Zezinho, que sempre me apoiaram, me escutaram e com os quais sei que sempre poderei contar. A Victória pelo enorme carinho.

A minha cunhada Walny e minha sobrinha Mariana, pelos seus sorrisos.

Especialmente aos meus pais: Luiz e Neusa, aos meus sogros: Miguel e Clarice e ao meu esposo e cúmplice: Wesley, pelo incentivo, encorajamento e, principalmente, pela oportunidade de estudar e completar mais esta etapa em minha vida. Se cheguei até aqui, foi graças a eles! 



\title{
Lista de Símbolos
}

\author{
$\mathbb{R} \quad$ o corpo dos reais \\ $\mathbb{C} \quad$ o corpo dos complexos \\ $\cong \quad$ isomorfismo \\ $\simeq$ homeomorfismo \\ $\sim \quad$ igualdade com relação a ordem \\ $\times \quad$ produto cartesiano de espaços \\ $\wedge \quad$ produto vetorial \\ $\langle$,$\rangle \quad produto interno usual do \mathbb{R}^{n}$ \\ $T^{\perp} \quad$ complemento ortogonal de $T$ \\ $A^{\circ} \quad$ interior de $A$ \\ $\bar{A} \quad$ fecho de $A$ \\ $\partial A$ bordo (ou fronteira) de $A$ \\ Ker núcleo \\ $\widehat{m p} \quad$ secante \\ $d f(p) \quad$ derivada de $f$ no ponto $p$ \\ $T_{m} X \quad$ espaço tangente a $X$ no ponto $m$ \\ $N_{m} X \quad$ espaço normal a $X$ no ponto $m$ \\ $\operatorname{dim} X$ dimensão de $X$ \\ Sing $V$ conjunto dos valores singular da variedade $V$
}





\section{Abstract}

In the present dissertation we do a study of algebraic, semialgebraic, analytic, semianalytic and subanalytic sets, real and complex, through the regularity conditions of the stratification of these sets. The basic idea in stratification is to decompose a singular space into manifolds; and the regularity conditions, is a control of how these manifolds fit together.

We do a general approach of the main regularity conditions. The conditions $(a)$ and $(b)$ of H. Whitney, the $(c)$-regularity of K. Bekka, the condition weakly Whitney, defined for D. Trotman and K. Bekka, the Kuo ratio test and the $(w)$-regularity of Verdier, presenting their main properties, theorems and conditions of existence. 



\section{Resumo}

Na presente dissertação faremos um estudo dos conjuntos algébricos, semialgébricos, analíticos, semianalíticos e subanalíticos, real e complexo, através das condições de regularidade da estratificação destes conjuntos. A idéia básica em estratificação é decompor um espaço singular em variedades regulares; e as condições de regularidade, são um controle de como essas variedades se reencontram.

Faremos uma abordagem geral das principais condições de regularidade. As condições $(a)$ e (b) de H. Whitney, a (c)-regularidade de K. Bekka, a condição Whitney fraca, definida por D. Trotman e K. Bekka, o teste da razão de Kuo e a $(w)$-regularidade de Verdier, apresentando suas principais propriedades, teoremas e condições de existência. 



\section{Sumário}

$\begin{array}{ll}\text { Introdução } & 1\end{array}$

1 Preliminares 3

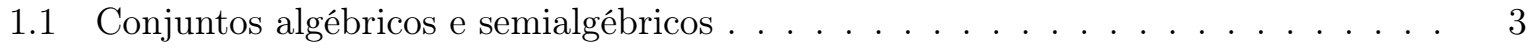

1.2 Conjuntos analíticos, semianalíticos e subanalíticos . . . . . . . . . . 6

1.3 Estratificações e exemplos . . . . . . . . . . . . . . . . . . . . 9

1.4 Condição de fronteira e Condição $(a)$ de H. Whitney . . . . . . . . . . . . . . 11

2 Teoria de estratificação de Thom-Whitney 15

2.1 A $(b)$-regularidade de H. Whitney . . . . . . . . . . . . . . . . . 15

2.2 Resultados e Exemplos . . . . . . . . . . . . . . . . . . 16

2.3 Propriedades dos espaços Whitney estratificados . . . . . . . . . . . . 26

3 Condição de regularidade de K. Bekka e Condição Whitney fraca 31

3.1 A $(c)$-regularidade de K. Bekka . . . . . . . . . . . . . . . . . 31

3.2 Estratificação $(c)$-regular de conjuntos subanalíticos . . . . . . . . . . . . . . 33

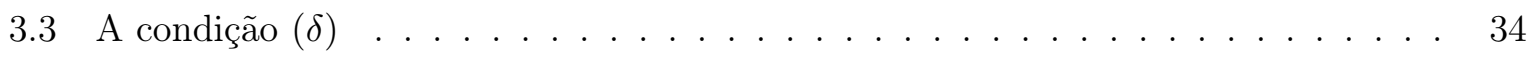

3.4 Estratificações Whitney fraca . . . . . . . . . . . . . . . . . 35

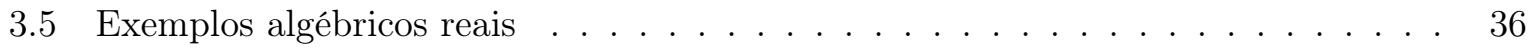

3.6 Propriedades dos espaços com uma estratificação Whitney fraca . . . . . . . . . . 40

4 Comparando a (b)-regularidade de Whitney com o teste da razão de Kuo 43

4.1 Teste da Razão de Kuo . . . . . . . . . . . . . . . . . . . . . . . . . . . . . . . 43

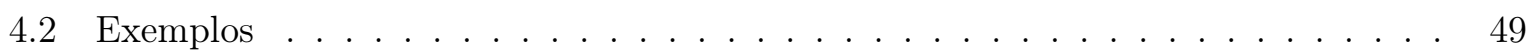

4.3 A $(w)$-regularidade de Verdier . . . . . . . . . . . . . . . . . . 52

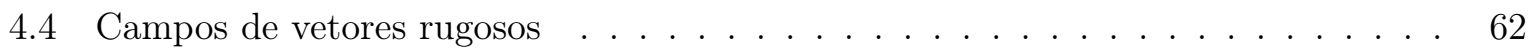

$\begin{array}{lr}\text { Bibliografia } & 66\end{array}$ 


\section{Introdução}

Um dos objetivos fundamentais da teoria de singularidades de aplicações é estudar a estrutura local de uma aplicação suave (isto é, infinitamente diferenciável), a qual pode ser extremamente complicada. De fato, qualquer subconjunto fechado do espaço euclideano é o conjunto de zeros de alguma função suave a valores reais (Teorema de Urysohn diferenciável). É necessário, portanto, impor algumas hipóteses genéricas sobre as aplicações em consideração. Por exemplo, se $M$ é uma variedade regular compacta, então as ferramentas elementares da Teoria de Morse [18] apresenta uma descrição completa da estrutura local de uma função a valores reais em $M$, quando os pontos críticos são não-degenerados. Além disso, é possível provar que essas funções formam um conjunto aberto e denso no espaço das funções suaves em $M$.

No estudo de singularidades de conjuntos, a principal idéia é utilizar a teoria de estratificação. Intuitivamente, podemos pensar numa estratificação como uma decomposição de um espaço singular em variedades regulares, chamados estratos, com algum tipo de controle de como estes estratos se reencontram. Tal controle é chamado condição de regularidade.

Em 1957, Whitney [26] mostrou que toda variedade algébrica $V=f^{-1}(0)$, onde $f: \mathbb{R}^{n} \longrightarrow \mathbb{R}^{p}$ tem coordenadas polinomiais, pode ser particionada em um número finito de variedades regulares conexas. Tal partição é obtida mostrando que a parte singular de $V$ é ainda algébrica, e de dimensão estritamente menor que $V$. Obtem-se, assim, uma filtração de $V$ por subvariedades algébricas da seguinte forma:

$$
V \supset \operatorname{Sing}(V) \supset \operatorname{Sing}(\operatorname{Sing}(V)) \supset \cdots
$$

Considerando uma aplicação $g: \mathbb{R}^{m} \longrightarrow \mathbb{R}^{n}$ suave, Thom propôs que deve existir uma estratificação de $V=f^{-1}(0)$ para a qual a condição de transversalidade de $g$ com relação aos estratos fosse uma condição aberta no conjunto das aplicações $C^{\infty}\left(\mathbb{R}^{m}, \mathbb{R}^{n}\right)$ e para isso, ele propôs que deveria existir alguma "trivialidade local" numa vizinhança de cada estrato. Em conseqüência, Whitney refinou sua definição em 2 artigos, [27] e [28], que surgiram em 1965, a respeito de estratificações de variedades analíticas reais e complexas. Thom, então, desenvolveu uma teoria de conjuntos $C^{\infty}$ estratificados, descrita em detalhes em 1969 no artigo intitulado "Ensembles et morphismes stratifiés" [21].

Assim, surgiu o que conhecemos por estratificação de Whitney (devida a Thom e Whitney).

Thom e Mather provaram um resultado assegurando a trivialidade topológica local ao longo 
dos estratos de uma estratificação de Whitney. Consequentemente, fazia-se a seguinte pergunta: Trivialidade topológica local implica estratificação de Whitney?

Em 1975, J. Briançon e J. P. Speder, apresentaram um exemplo que admitia uma estratificação assegurando a trivialidade topológica local, e esta estratificação não era uma estratificação de Whitney.

Em 1991, K. Bekka definiu uma outra condição de regularidade, denominada (c)-regular. Tal condição, apesar de ser mais fraca que a condição $(b)$ de Whitney, ainda implica trivialidade topológica local.

Na presente dissertação, faremos uma abordagem geral das principais condições de regularidade, a saber, as condições (a) e (b) de H. Whitney [15] e [28], a (c)-regularidade de K. Bekka, a condição Whitney fraca, o teste da razão de Kuo e a $(w)$-regularidade de Verdier, apresentando suas principais propriedades, teoremas e condições de existência.

Nosso objetivo é fazer uma comparação entre essas condições de regularidade. Não apresentaremos estas ferramentas de um ponto de vista histórico; o que tentaremos aqui é apresentálas numa seqüência que entendemos ser mais esclarecedora para o leitor que está tendo um primeiro contato com a teoria.

No capítulo 1, definiremos os conjuntos algébricos e semialgébricos e apresentaremos alguns resultados e propriedades desses conjuntos. Na mesma linha de definição dos conjuntos algébricos e semialgébricos definiremos os conjuntos analíticos, semianalíticos e subanalíticos. Além disso, introduziremos a condição de fronteira e a condição $(a)$-regular de Whitney.

No capítulo 2, apresentaremos alguns resultados básicos da teoria de estratificação de ThomWhitney, dentre eles, resultados que mostram como a estratificação de Whitney nos ajuda a estudar a estrutura local de uma aplicação suave. Ainda no mesmo capítulo, introduziremos o resultado que garante a trivialidade topológica local em uma estratificacão de Whitney.

No capítulo 3, introduziremos a (c)-regularidade definida por K. Bekka em [1], a qual implica trivialidade topológica local, e verificaremos que essa condição é mais fraca que a condição $(b)$ de Whitney. Ainda no mesmo capítulo, apresentaremos a condição Whitney fraca, definida por D. Trotman e K. Bekka em [3], e mostraremos que estes espaços formam uma classe intermediária entre os espaços Whitney estratificados e os espaços $(c)$-regulares.

No capítulo 4, apresentaremos e provaremos o teste da razão de Kuo [11]. Além disso, introduziremos a condição de regularidade $(w)$ de Verdier, definida em [24], a qual apresenta um papel muito importante no estudo de conjuntos algébricos e analíticos. Ainda no mesmo capítulo, mostraremos as diferenças entre as condições (b) de Whitney e o teste da razão de Kuo para estratificações em que o estrato de menor dimensão é ao menos 2-dimensional. 


\section{Capítulo 1}

\section{Preliminares}

Neste capítulo inicial, definiremos e apresentaremos alguns resultados e propriedades dos conjuntos algébricos, semialgébricos, analíticos, semianalíticos e subanalíticos. Mais detalhes podem ser encontrados em [4], [5] e [19]. Além disso, definiremos estratificação de conjuntos e introduziremos dois importantes conceitos, a saber, a condição de fronteira e a condição $(a)$ de Whitney.

\subsection{Conjuntos algébricos e semialgébricos}

Definição 1.1. Um conjunto $A \subset \mathbb{R}^{n}$ é dito algébrico, quando existe uma função polinomial $f: \mathbb{R}^{n} \longrightarrow \mathbb{R}$, tal que, $A=\left\{x \in \mathbb{R}^{n} ; f(x)=0\right\}$, ou seja, $A=f^{-1}(0)$.

Exemplo 1.1. Seja $S^{n}=\left\{x=\left(x_{1}, \ldots, x_{n+1}\right) \in \mathbb{R}^{n+1} ; \sum_{i=1}^{n+1} x_{i}^{2}=1\right\}$. Então, se tomarmos $f(x)=\sum_{i=1}^{n+1} x_{i}^{2}-1$, claramente temos que $S^{n}=f^{-1}(0)$.

Exemplo 1.2. Seja $A=\left\{(x, 0) \in \mathbb{R}^{2} ; x \in \mathbb{R}\right\} \cup\left\{(0, y) \in \mathbb{R}^{2} ; y \in \mathbb{R}\right\}$. Basta considerarmos $f(x)=x y$, para obtermos $A=f^{-1}(0)$.

Proposição 1.1. Sejam $A, B$ conjuntos algébricos em $\mathbb{R}^{n}$. Então $A \cup B$ e $A \cap B$ são conjuntos algébricos.

Demonstração: Como $A, B$ são conjuntos algébricos, existem $f_{1}$ e $f_{2}$ funções polinomiais, tais que, $A=f_{1}^{-1}(0)$ e $B=f_{2}^{-1}(0)$. Claramente $A \cup B=\left(f_{1} \cdot f_{2}\right)^{-1}(0)$ e $A \cap B=\left(f_{1}^{2}+f_{2}^{2}\right)^{-1}(0)$. Agora, como $g=f_{1} \cdot f_{2}$ e $h=f_{1}^{2}+f_{2}^{2}$ ainda são funções polinomiais, o resultado segue.

Proposição 1.2. Sejam $B \subset \mathbb{R}^{n}$ um conjunto algébrico e $F: \mathbb{R}^{m} \longrightarrow \mathbb{R}^{n}$ uma aplicação polinomial. Então $F^{-1}(B) \subset \mathbb{R}^{m}$ é um conjunto algébrico.

Demonstração: Por hipótese, existe uma função polinomial $\phi: \mathbb{R}^{n} \longrightarrow \mathbb{R}$ tal que $B=\phi^{-1}(0)$. Sabemos que $F^{-1}(B)=\left\{x \in \mathbb{R}^{n} ; \exists y \in B, F(x)=y\right\}$. Assim, para todo $x \in F^{-1}(B), \exists y \in B$ 
tal que $\phi(F(x))=\phi(y)=0$, ou seja, $F^{-1}(B)=(\phi \circ F)^{-1}(0)$. Como $\phi \circ F$ é ainda uma função polinomial, temos que $F^{-1}(B)$ é um conjunto algébrico.

A proposição anterior nos leva a seguite dúvida: A imagem de um conjunto algébrico por uma aplicação polinomial é um conjunto algébrico? Isso nem sempre é verdade. Vejamos um exemplo:

Exemplo 1.3. Sejam $\pi: \mathbb{R}^{2} \longrightarrow \mathbb{R}$ a projeção $\pi(x, y)=x$ e $S^{1}=\left\{(x, y) \in \mathbb{R}^{2} ; x^{2}+y^{2}=1\right\}$. Vimos que $S^{1}$ é um conjunto algébrico, mas $\pi\left(S^{1}\right)=[-1,1]$ não o é. De fato, para todo polinômio $f: \mathbb{R} \longrightarrow \mathbb{R}$, temos duas possibilidades para $f^{-1}(0)$. Se $f$ não for o polinômio identicamente nulo, temos que $f^{-1}(0)$ é o conjunto finito de raízes do polinômio. Caso contrário, $f^{-1}(0)$ é toda reta, logo não existe um polinômio $f$, tal que, $f^{-1}(0)=[-1,1]$. Ou seja, o intervalo $[-1,1]$ não é um conjunto algébrico.

Este exemplo motiva a definição de uma classe mais geral que a dos conjuntos algébricos, a saber, a classe dos conjuntos semialgébricos.

Definição 1.2. Um conjunto $A \subset \mathbb{R}^{n}$ é dito semialgébrico básico quando existem $f, g_{1}, \ldots, g_{k}$ funções polinomiais definidas em $\mathbb{R}^{n}$, tais que:

$$
A=\left\{x \in \mathbb{R}^{n} ; f(x)=0\right\} \cap\left(\bigcap_{i=1}^{k}\left\{x \in \mathbb{R}^{n} ; g_{i}(x)>0\right\}\right) .
$$

Dizemos que $f, g_{1}, \ldots, g_{k}$ definem o conjunto $A$. Observe que se $f$ é a função polinomial identicamente nula, apenas $g_{1}, \ldots, g_{k}$ definem o conjunto $A$.

Definição 1.3. Um conjunto semialgébrico é a reunião finita de conjuntos semialgébricos básicos. Ou seja, se $A \subset \mathbb{R}^{n}$ é semialgébrico existem $f_{i}, g_{i j}$ funções polinomiais definidas em $\mathbb{R}^{n}$, tais que:

$$
A=\bigcup_{i=1}^{k}\left(\left\{x \in \mathbb{R}^{n} ; f_{i}(x)=0\right\} \bigcap\left(\bigcap_{j=1}^{s_{i}}\left\{x \in \mathbb{R}^{n} ; g_{i j}(x)>0\right\}\right)\right) .
$$

Obviamente todo conjunto algébrico é semialgébrico.

Proposição 1.3. Um conjunto finito de pontos em $\mathbb{R}^{n}$ é um conjunto semialgébrico.

Demonstração: Seja $A=\left\{y_{1}, \ldots, y_{k}\right\} \subset \mathbb{R}^{n}$. E, para cada $y_{i}=\left(y_{i_{1}}, \ldots, y_{i_{m}}\right)$, seja $f_{i}(x)=\sum_{j=1}^{m}\left(x_{j}-y_{i_{j}}\right)^{2}$. Então, $A=\bigcup_{i=1}^{k}\left\{x \in \mathbb{R}^{n} ; f_{i}(x)=0\right\}$ é, claramente, um conjunto semialgébrico.

Proposição 1.4. Na reta, um conjunto semialgébrico é a reunião finita de intervalos abertos, fechados, semiabertos e pontos.

Demonstração: Seja $A \subset \mathbb{R}$ um conjunto semialgébrico básico. Então, existem $f, g_{1}, \ldots, g_{k}$ polinômios reais que definem o conjunto $A$. Se $f$ não for o polinômio identicamente nulo, certamente $A$ é um conjunto finito que é, no máximo, as raízes de $f$. Agora, se $f$ for o polinômio 
identicamente nulo então, $g_{1}, \ldots, g_{k}$ definem $A$. Temos que, $A_{j}=\left\{x \in \mathbb{R} ; g_{j}(x)>0\right\}$ é reunião de intervalos abertos, limitados ou não, cujos extremos são raízes consecutivas de $g_{j}$, ou seja, $A_{j}$ é reunião de intervalos abertos do tipo $(-\infty, a),(a, b)$ ou $(b,+\infty)$, onde $a$ e $b$ são raízes consecutivas de $g_{j}$. A interseção de conjuntos desse tipo é ainda um conjunto desse tipo, sendo que os extremos dos intervalos podem ser raízes diferentes de $g_{j}$. Como um conjunto semialgébrico é a união de conjuntos semialgébricos básicos, temos que todo conjunto semialgébrico em $\mathbb{R}$ é a união finita de intervalos abertos, fechados, semiabertos e pontos.

Definição 1.4. Seja $A \subset \mathbb{R}^{n}$. Dizemos que a função $F: A \longrightarrow \mathbb{R}$ é semialgébrica se seu gráfico $\operatorname{Graf}(F)=\{(x, F(x)) ; x \in A\} \subset \mathbb{R}^{n+1}$ é um conjunto semialgébrico.

Exemplo 1.4. Seja $h: \mathbb{R}_{+} \longrightarrow \mathbb{R}$ uma função definida por $h(x)=\sqrt{x}$. Então, temos que $\operatorname{Graf}(h)=\left\{(x, y) \in \mathbb{R}^{2} ; y^{2}=x, y \geq 0\right\}$. Tomando $f(x, y)=y^{2}-x, g_{1}(x, y)=y$ e unindo o semialgébrico básico definido por $f, g_{1}$ a origem $(0,0)$, segue diretamente da definição que Graf $(h)$ é um conjunto semialgébrico, logo $h$ é uma função semialgébrica.

Enunciaremos agora um importante teorema sobre conjuntos semialgébricos.

Teorema 1.1. (Teorema da decomposição cilíndrica, [19], pág.7-8): Seja $X \subset \mathbb{R}^{n+1}$ um conjunto semialgébrico. Então:

(i) X possui um número finito de componentes conexas, onde cada componente é ainda um conjunto semialgébrico.

(ii) existe uma partição finita $A_{1}, \ldots, A_{k}$, de conjuntos semialgébricos e conexos do $\mathbb{R}^{n}$ e para cada $A_{i}$, um conjunto finito de funções continuas $f_{j}: A_{i} \longrightarrow \mathbb{R} ; f_{0}=-\infty, f_{j}>f_{j-1}$ e $f_{s}=+\infty$, tais que, $X \cap G_{i j}=G_{i j}$ ou vazio e $X \cap B_{i j}=B_{i j}$ ou vazio, para todo $i=1, \ldots, k$ e $j=1, \ldots, s$, onde $G_{i j}=\left\{\left(x, f_{j}(x)\right) ; x \in A_{i}\right\}$ são conjuntos tipo gráfico $e$ $B_{i j}=\left\{(x, t) ; x \in A_{i}, f_{j}(x)<t<f_{j+1}(x)\right\}$ são conjuntos tipo faixa.

Citaremos ao longo deste capítulo, alguns resultados importantes que são conseqüências do Teorema da decomposição cilíndrica. Como por exemplo:

Teorema 1.2. (Tarski-Seidenberg, [19], pág.8-9): A imagem de um conjunto semialgébrico $A \subset \mathbb{R}^{n+1}$ por uma projeção $\pi: \mathbb{R}^{n+1} \longrightarrow \mathbb{R}^{n}$ própria é um conjunto semialgébrico.

Demonstração: pelo Teorema da decomposição cilíndrica

$$
A=\bigcup_{i=1}^{k}\left(\bigcup_{j=1}^{s}\left(G_{i j} \cap B_{i j}\right)\right),
$$

onde $\pi\left(G_{i j}\right)=\pi\left(B_{i j}\right)=A_{i}$. Ainda pelo Teorema da decomposição cilíndrica, cada $A_{i}$ é um conjunto semialgébrico então, $\pi(A)$ é reunião finita de conjuntos semialgébricos e, consequentemente, é semialgébrico.

Temos agora válido o seguinte resultado: 
Corolário 1.1.1. Sejam $X \subset \mathbb{R}^{n}$ um conjunto semialgébrico e $F: \mathbb{R}^{n} \longrightarrow \mathbb{R}^{m}$ uma aplicação polinomial. Então $F(X)$ é um conjunto semialgébrico.

Definição 1.5. Sejam $A \subset \mathbb{R}^{n}$ e $B \subset \mathbb{R}^{m}$. Uma aplicação $F: A \longrightarrow B$ é semialgébrica se suas funções componentes são semialgébricas ou, equivalentemente, se seu gráfico $G r a f(F) \subset \mathbb{R}^{n+m}$ é um conjunto semialgébrico.

Corolário 1.1.2. Sejam $f: A \subset \mathbb{R}^{p} \longrightarrow \mathbb{R}^{n}$ e $g: B \subset \mathbb{R}^{n} \longrightarrow \mathbb{R}^{m}$ aplicações semialgébricas com $f(A) \subset B$. Então $g \circ f: A \subset \mathbb{R}^{p} \longrightarrow \mathbb{R}^{m}$ é uma aplicação semialgébrica.

Demonstração: Por hipótese, temos que $f(A) \subset B, \operatorname{Graf}(f)=\left\{(x, f(x)) \in \mathbb{R}^{p} \times \mathbb{R}^{n} ; x \in A\right\}$ e $\operatorname{Graf}(g)=\left\{(y, g(y)) \in \mathbb{R}^{n} \times \mathbb{R}^{m} ; y \in B\right\}$ são conjuntos semialgébricos; logo,

$$
L=\left\{(x, f(x), g(f(x))) \in \mathbb{R}^{p} \times \mathbb{R}^{n} \times \mathbb{R}^{m} ; x \in A\right\}
$$

é também semialgébrico. Tomando a projeção $\pi: \mathbb{R}^{p} \times \mathbb{R}^{n} \times \mathbb{R}^{m} \longrightarrow \mathbb{R}^{p} \times \mathbb{R}^{m}$, dada por $\pi(x, y, z)=(x, z)$, temos que Graf $(g \circ f)=\pi(L)$. Pelo Teorema de Tarski-Seidenberg $\pi(L)$ é um conjunto semialgébrico. Logo, $g \circ f$ é uma aplicação semialgébrica.

Exemplo 1.5. Seja $f: \mathbb{R}^{n} \longrightarrow \mathbb{R}$ dada por $f(x)=\|x\|$. Sabemos que $\|x\|=\sqrt{\sum_{i=1}^{n} x_{i}^{2}}$. Vimos também que $g(y)=\sqrt{y}$ é uma função semialgébrica, segue então, do corolário acima, que $\|x\|$ é uma função semialgébrica.

Algumas propriedades de conjuntos semialgébricos:

(i) Seja $A \subset \mathbb{R}^{n}$ um conjunto semialgébrico. Então o fecho, o interior e a fronteira de $A$ são conjuntos semialgébricos;

(ii) Sejam $A, B \subset \mathbb{R}^{n}$ conjuntos semialgébricos. Então, $A \cup B, A \cap B, A \backslash B, B \backslash A, \mathbb{R}^{n} \backslash A \mathrm{e}$ $\mathbb{R}^{n} \backslash B$ são conjuntos semialgébricos;

(iii) Sejam $A \subset \mathbb{R}^{n}$ e $B \subset \mathbb{R}^{m}$ conjuntos semialgébricos. Então $A \times B \subset \mathbb{R}^{n} \times \mathbb{R}^{m}$ é um conjunto semialgébrico.

\subsection{Conjuntos analíticos, semianalíticos e subanalíticos}

Definição 1.6. Um conjunto $A \subset \mathbb{R}^{n}$ é dito analítico se para todo $x \in A$ existe uma vizinhança $U_{x} \subset \mathbb{R}^{n}$ de $x$ e uma função analítica $f: U_{x} \longrightarrow \mathbb{R}$, tal que, $A \cap U_{x}=f^{-1}(0)$.

Relembremos aqui que uma função $f: U \subset \mathbb{R}^{n} \longrightarrow \mathbb{R}$ é analítica se para todo $x_{0} \in U$ existe um aberto $U_{0} \subset \mathbb{R}^{n}$ onde $f$ pode ser desenvolvida como série de potências.

Exemplo 1.6. Seja $f: \mathbb{R}^{2} \longrightarrow \mathbb{R}$ definida por $f(x, y)=e^{x}+\sin x^{2}+y^{3}$. Então o conjunto $A=\left\{(x, y) \in \mathbb{R}^{2} ; f(x, y)=0\right\}$ é um conjunto analítico. 
Claramente, todo polinômio é uma aplicação analítica; logo, todo conjunto algébrico é um conjunto analítico.

Definição 1.7. Um subconjunto $A \subset \mathbb{R}^{n}$ é dito semianalítico básico se para todo $x \in A$ existe uma vizinhança $U_{x} \subset \mathbb{R}^{n}$ de $x$ e funções $f, g_{1}, \ldots, g_{k}$ analiticas em $U_{x}$ tal que:

$$
A \cap U_{x}=\left\{x \in \mathbb{R}^{n} ; f(x)=0\right\} \cap\left(\bigcap_{i=1}^{k}\left\{x \in \mathbb{R}^{n} ; g_{i}(x)>0\right\}\right) .
$$

Definição 1.8. Um conjunto semianalítico é a reunião finita de conjuntos semianalíticos básicos.

Exemplo 1.7. Seja $f: \mathbb{R}^{2} \longrightarrow \mathbb{R}$ definida por $f(x, y)=e^{x}-y$; então, o conjunto definido por $A=\left\{(x, y) \in \mathbb{R}^{2} ; f(x, y)>0\right\}$ é um conjunto semianalítico.

Observação 1.1. Devemos prestar atenção nos pontos que pertencem a $\bar{A} \backslash A$. Note, por exemplo, que o conjunto $y=\sin \frac{1}{x}, x \neq 0$, no $\mathbb{R}^{2}$ não é semianalítico, visto que os pontos que pertencem a $\bar{A} \backslash A$ (que são da forma $(0, y)$, com $|y| \leq 1$ ) não tem a propriedade requerida. Mas todos os outros pontos tem esta propriedade.

Da mesma forma que todo conjunto algébrico é semialgébrico, fica claro que todo conjunto analítico é um conjunto semianalítico. E como todo polinômio é analítico, temos que, todo conjunto semialgébrico é um conjunto semianalítico.

Definição 1.9. Seja $A \subset \mathbb{R}^{n}$. Dizemos que a função $F: A \longrightarrow \mathbb{R}$ é semianalítica se seu gráfico $\operatorname{Graf}(F)=\{(x, F(x)) ; x \in A\} \subset \mathbb{R}^{n+1}$ é um conjunto semianalítico.

Definição 1.10. Sejam $A \subset \mathbb{R}^{n}$ e $B \subset \mathbb{R}^{m}$. Uma aplicação $F: A \longrightarrow B$ é semianalítica se suas funções componentes são semianalíticas ou, equivalentemente, se seu gráfico Graf $(F) \subset \mathbb{R}^{m+n}$ é um conjunto semianalítico.

É importante salientar o caráter local dessas definições em contrapartida à definição de conjuntos algébricos e semialgébricos. Mas, a semelhança no modo de se definir esses conjuntos justifica o fato de que muitos dos resultados válidos para conjuntos semialgébricos também são válidos para conjuntos semianalíticos. Porém, nem todos resultados são válidos e, infelizmente, o importante Teorema de Tarski-Seidenberg não é válido. Assim, não é válido que aplicações analíticas levam conjuntos semianalíticos em conjuntos semianalíticos. Resultado este que seria conseqüência do Teorema de Tarski-Seidenberg, ou seja, o Teorema de Tarski-Seidenberg não vale para conjuntos semianalíticos.

Isso motiva a definição de uma categoria mais geral que semianalíticos, a saber, os conjuntos subanalíticos.

Definição 1.11. Um subconjunto $A \subset \mathbb{R}^{n}$ é dito subanalitico se existe um conjunto $\widetilde{A} \subset \mathbb{R}^{m}$, semianalítico, $m \geq n$, tal que a projeção $\pi: \mathbb{R}^{m} \longrightarrow \mathbb{R}^{n}$ restrita a $\widetilde{A}$ é uma aplicação própria e $A=\pi(\widetilde{A})$. 
Com esta definição torna-se naturalmente válido o Teorema de Tarski-Seidenberg. De fato, sejam $A \subset \mathbb{R}^{n}$ um conjunto subanalítico e $\pi: \mathbb{R}^{n} \longrightarrow \mathbb{R}^{k}, n \geq k$, uma projeção. Sabemos que existe um conjunto semianalítico $\widetilde{A} \subset \mathbb{R}^{m}, m \geq n$, tal que a projeção $\widetilde{\pi}: \mathbb{R}^{m} \longrightarrow \mathbb{R}^{n}$ restrita a $\widetilde{A}$ é uma aplicação própria e $A=\widetilde{\pi}(\widetilde{A})$. Logo, $\widetilde{\pi} \circ \pi$ define $B=\pi(A)$ como conjunto subanalítico.

Obviamente, todo conjunto semianalítico é subanalítico.

Como antes, temos as seguintes definições:

Definição 1.12. Seja $A \subset \mathbb{R}^{n}$. Dizemos que a função $F: A \longrightarrow \mathbb{R}$ é subanalítica se seu gráfico $\operatorname{Graf}(F)=\{(x, F(x)) ; x \in A\} \subset \mathbb{R}^{n+1}$ é um conjunto subanalítico.

Definição 1.13. Sejam $A \subset \mathbb{R}^{n}$ e $B \subset \mathbb{R}^{m}$. Uma aplicação $F: A \longrightarrow B$ é subanalítica se suas funções componentes são subanalíticas ou, equivalentemente, se seu gráfico $\operatorname{Graf}(F) \subset \mathbb{R}^{m+n}$ é um conjunto subanalitico.

Algumas propriedades de conjuntos subanalíticos:

(i) Se $A_{1}$ e $A_{2}$ são conjuntos subanalíticos, então $A_{1} \cup A_{2}$ é um conjunto subanalítico;

(ii) (Teorema de Gabrielov) Se $A \subset \mathbb{R}^{n}$ é um conjunto subanalítico, então $\mathbb{R}^{n} \backslash A$ é um conjunto subanalítico;

Como conseqüência de (i) e (ii), temos que, interseção e diferença de conjuntos subanalíticos é um conjunto subanalítico.

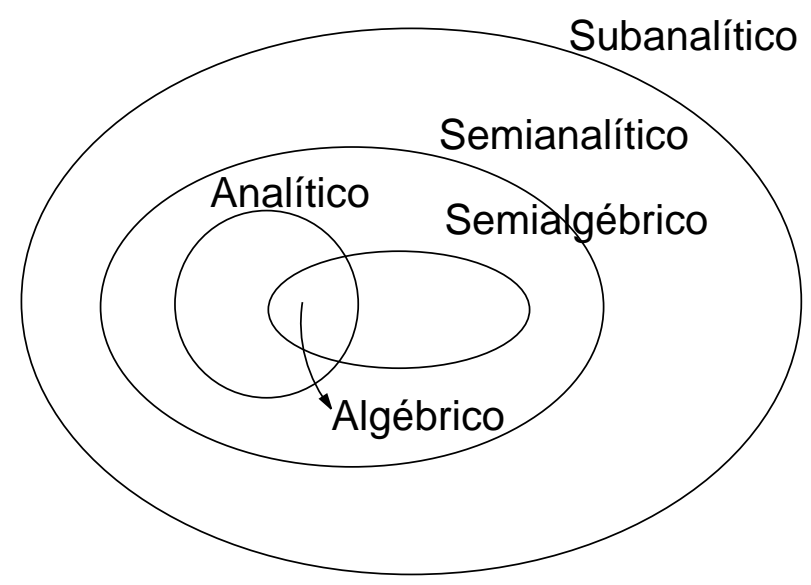

Figura 1.1: Diagrama

(iii) Se $F: A \subset \mathbb{R}^{m} \longrightarrow \mathbb{R}^{n}$ é uma aplicação subanalítica, então $A$ e $F(A)$ são conjuntos subanalíticos;

(iv) Se $A \subset \mathbb{R}$ é um conjunto subanalítico, então $A$ é reunião finita de intervalos abertos, fechados, semiabertos e pontos;

(v) Composta de funções subanalíticas ainda é uma função subanalítica; 
(vi) Seja $A \subset \mathbb{R}^{n}$. Então, o fecho, o interior e a fronteira de $A$, denotados respectivamente por $\bar{A}, A^{\circ}$ e $\partial A$, são conjuntos subanalíticos, bem como o complementar e o produto cartesiano de conjuntos subanalíticos ainda são conjuntos subanalíticos.

Teorema 1.3. (Uniformização, [4],[5]): Seja M uma variedade regular analítica real. Suponha que A é um subconjunto subanalítico fechado de $M$. Então existe uma variedade regular analítica real $N$ (com mesma dimensão de A) e uma aplicação analítica real própria $\varphi: N \longrightarrow M$ tal que $\varphi(N)=A$.

Agora, enunciaremos um lema que será muito utilizado ao longo da dissertação.

Lema 1.1. (Lema de seleção da curva, [17], §3, pág. 25): Se A é um conjunto semianalítico do $\mathbb{R}^{n}$ e $p \in \bar{A} \backslash A$, então podemos selecionar uma curva analítica $a(s)=\left(x_{1}(s), \ldots, x_{n}(s)\right.$ ) (isto é, cada $x_{i}(s)$ é uma série de potência convergente) tal que $a(0)=p$, e a $(s) \in A$ se $s \neq 0$.

\subsection{Estratificações e exemplos}

A idéia básica na teoria de estratificação é decompor um espaço singular em variedades regulares, que chamamos de estratos, de tal forma que cada estrato consiste de pontos igualmente "ruins", num certo sentido. Nesta seção, estaremos apresentando alguns exemplos de estratificações.

Definição 1.14. Uma estratificação de uma variedade $V$ é uma decomposição de $V$ como a união finita e disjunta de variedades regulares sem bordo, abertas ou fechadas, $V=X_{1} \cup \ldots \cup X_{p}$.

Vejamos abaixo alguns exemplos de estratificações:

Exemplo 1.8. Seja $f_{1}(x, y, z)=z\left(x^{2}-y^{2}\right)+x^{4}+y^{4}$; então, a variedade $V_{1}$ definida por $f_{1}$ é $V_{1}=\left\{(x, y, z) \in \mathbb{R}^{3} ; f_{1}(x, y, z)=0\right\}$. Temos as seguintes estratificações:

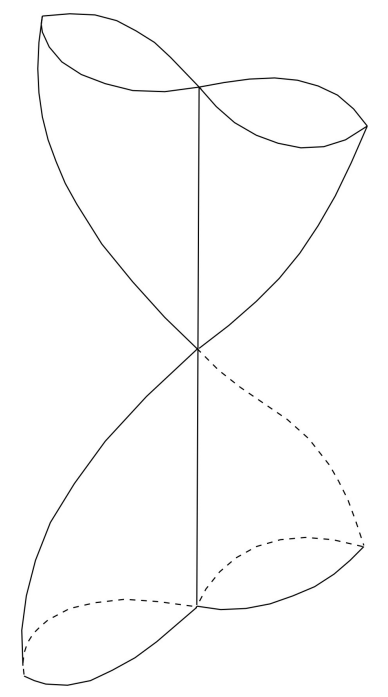

Figura 1.2: $V_{1}$

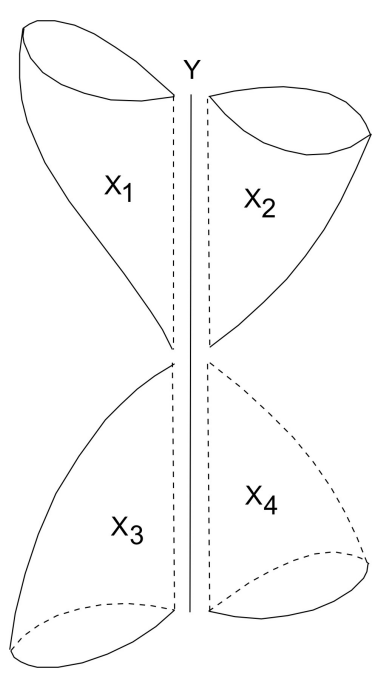

Figura 1.3: Estrat.1

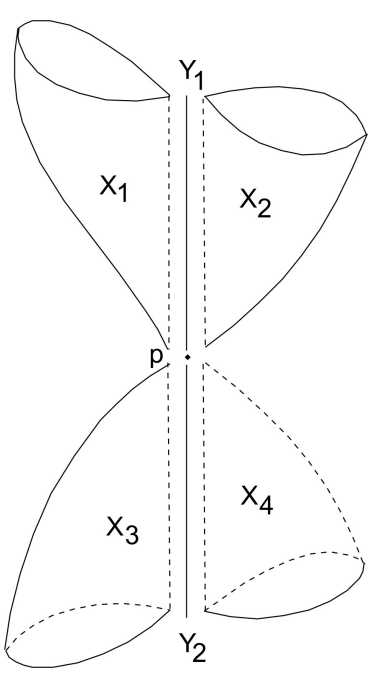

Figura 1.4: Estrat.2 
Exemplo 1.9. Seja $f_{2}(x, y)=x\left(x-y^{2}\right)$; então, a variedade $V_{2}$ definida por $f_{2}$ é $V_{2}=\left\{(x, y) \in \mathbb{R}^{2} ; f_{2}(x, y)=0\right\}$. Aqui, temos as seguintes estratificações:

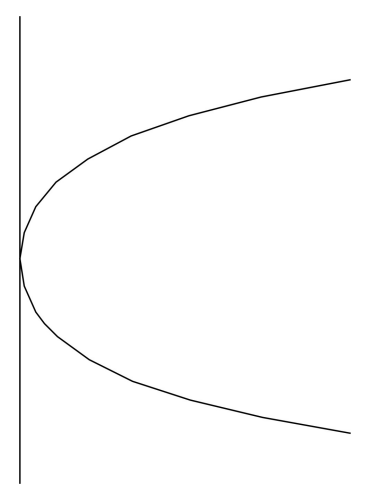

Figura 1.5: $V_{2}$

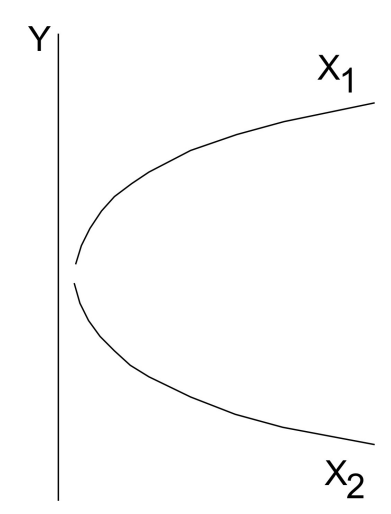

Figura 1.6: Estrat.1

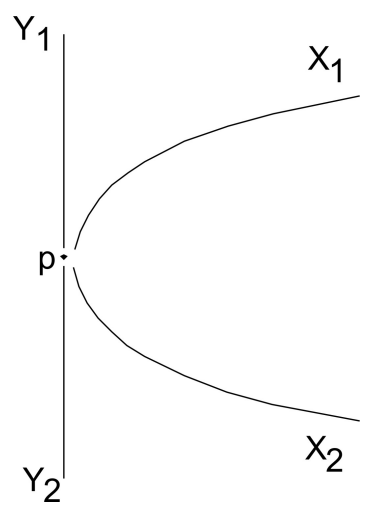

Figura 1.7: Estrat.2

Exemplo 1.10. (O "Guarda-chuva de Whitney") Seja $f_{3}(x, y, z)=x^{2}-y^{2} z$; então, a variedade $V_{3}$ definida por $f_{3}$ é $V_{3}=\left\{(x, y, z) \in \mathbb{R}^{3} ; x^{2}=y^{2} z\right\}$. Considere a seguinte estratificação:

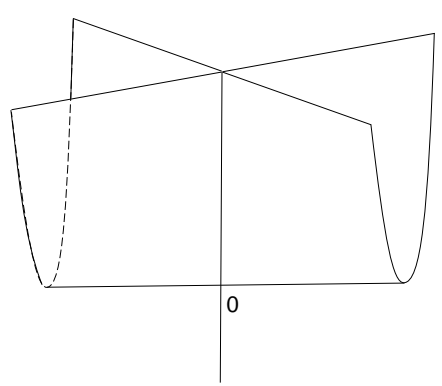

Figura 1.8: $V_{3}$

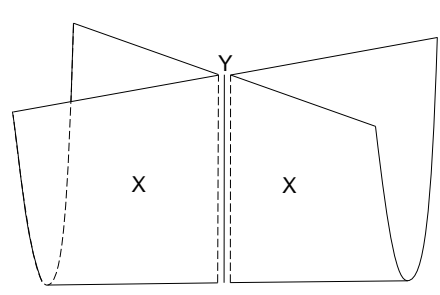

Figura 1.9: Estrat.1

Exemplo 1.11. ("Cúspide de Whitney") Seja $f_{4}(x, y, z)=y^{2}-z^{2} x^{2}-x^{3}$; então, a variedade $V_{4}$ definida por $f_{4}$ é $V_{4}=\left\{(x, y) \in \mathbb{R}^{3} ; f_{4}(x, y, z)=0\right\}$. Aqui, temos as seguintes estratificações:

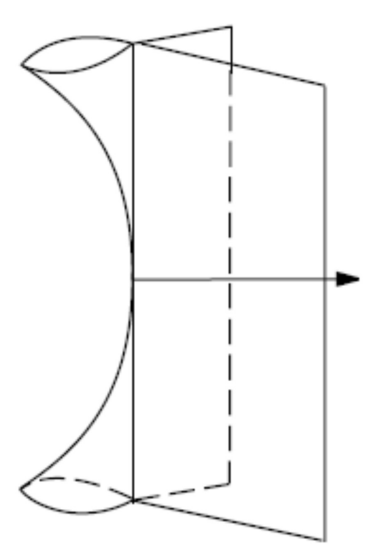

Figura 1.10: $V_{4}$

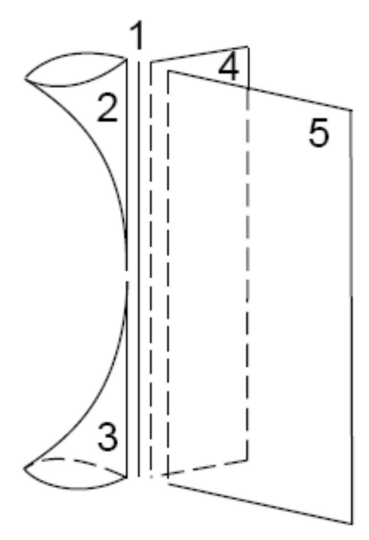

Figura 1.11: Estrat.1

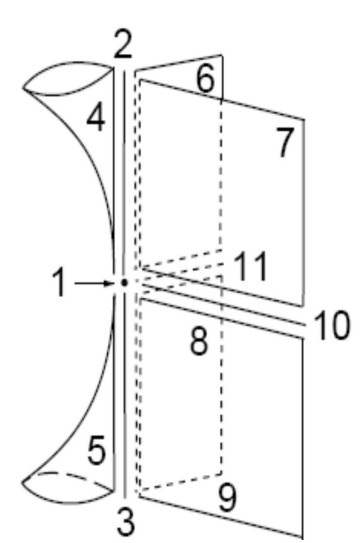

Figura 1.12: Estrat.2 
A noção mais importante na teoria de estratificação é uma condição de regularidade entre os estratos de uma estratificação. Essas condições indicarão a maneira pela qual separaremos os estratos em pontos igualmente "ruins".

\subsection{Condição de fronteira e Condição $(a)$ de H. Whitney}

Podemos pensar numa condição de regularidade como sendo um controle de como os estratos de uma estratificação se reencontram. Nesta seção, introduziremos dois conceitos principais na teoria de estratificação: a condição de fronteira e a condição de regularidade $(a)$ de Whitney.

Definição 1.15. Uma estratificação $V=X_{1} \cup \ldots \cup X_{p}$ satisfaz a condição de fronteira se, sempre que $X_{i} \cap \overline{X_{j}} \neq \emptyset$, tem-se que: $X_{i} \subset \overline{X_{j}}$.

Observação 1.2. Note que a estratificação de $V_{2}$ dada em Estrat.1 não satisfaz a condição de fronteira, visto que $Y \cap \overline{X_{i}} \neq \emptyset$ mas, $Y \not \subset \overline{X_{i}}, i=1,2$. Entretanto, as estratificações de $V_{4}$ dadas em Estrat.1 e Estrat.2, no exemplo 1.11, satisfazem a condição de fronteira.

Definição 1.16. Uma estratificação de $V=X_{1} \cup \ldots \cup X_{p}$ é dita algébrica, semialgébrica, analítica, semianalítica ou subanalítica, se cada variedade regular $X_{i}$ é um conjunto algébrico, semialgébrico, analítico, semianalítico ou subanalítico, respectivamente.

A seguir, definiremos a condição de regularidade $(a)$, antes porém, precisaremos fixar as seguintes notações:

Seja $V=X \cup Y \cup \ldots$ um subconjunto estratificado do $\mathbb{R}^{n}$, com estratos $X, Y, \ldots$.

Sejam $p \in Y, m \in X, T_{p} Y$ o espaço tangente a $Y$ no ponto $p$ e $N_{m} X$ o espaço normal a $X$ no ponto $m$. Denotemos por $\pi_{N_{m} X}(\tau)$ a projeção ortogonal de $\tau$ sobre $N_{m} X$.

Definição 1.17. Dizemos que $X$ é (a)-regular sobre $Y$ no ponto $p \in Y$, se para todo vetor tangente $\tau \in T_{p} Y$ tem-se:

$$
\lim _{m \rightarrow p} \pi_{N_{m} X}(\tau)=0
$$

Ou seja, o par $(X, Y)$ satisfaz a condição $(a)$ em $p$ se vale o seguinte: Dada qualquer seqüência $\left\{m_{i}\right\}$ de pontos em $X$ tal que $m_{i} \rightarrow p$ e $T_{m_{i}} X$ convirja para algum plano $\tau \subset \mathbb{R}^{n}$, temos que: $T_{p} Y \subset \tau$.

Definição 1.18. Dizemos que o par $(X, Y)$ satisfaz a condição (a) se ele satisfaz (a) em todo ponto de $Y$.

Vamos verificar a seguir, se as estratificações dadas nos exemplos 1.8, 1.9 e 1.11 são (a)-regulares.

No exemplo 1.8, temos que a variedade $V_{1}$ é definida por $V_{1}=\left\{(x, y, z) \in \mathbb{R}^{3} ; f_{1}(x, y, z)=0\right\}$, onde $f_{1}(x, y, z)=z\left(x^{2}-y^{2}\right)+x^{4}+y^{4}$; então temos as seguintes situações: 
(i) Estratificação de $V_{1}$ em Estrat.1:

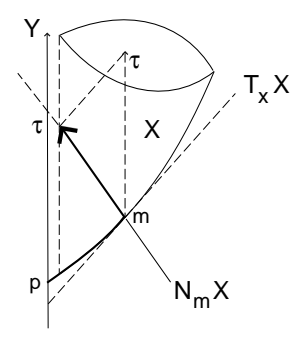

Figura 1.13:

Seja $p=(0,0,0) \in Y$. Para todo $m=(x, y, z) \in X,(x, y) \neq(0,0)$, temos que o espaço normal é gerado por:

$$
\nabla f_{1}(x, y, z)=\left(2 x z+4 x^{3},-2 y z+4 y^{3}, x^{2}-y^{2}\right)
$$

O vetor normal (direção) é:

$$
v(m)=\frac{\nabla f_{1}(x, y, z)}{\left\|\nabla f_{1}(x, y, z)\right\|}=\left(\frac{2 x z+4 x^{3}}{\sqrt{A_{1}}}, \frac{-2 y z+4 y^{3}}{\sqrt{A_{1}}}, \frac{x^{2}-y^{2}}{\sqrt{A_{1}}}\right)
$$

onde $A_{1}=\left(2 x z+4 x^{3}\right)^{2}+\left(-2 y z+4 y^{3}\right)^{2}+\left(x^{2}-y^{2}\right)^{2}$.

Além disso, se $\tau \in T_{p} Y$, podemos assumir que $\tau=(0,0,1)$, visto que $T_{p} Y$ é 1-dimensional.

Assim,

$\left|\pi_{N_{m} X}(\tau)\right|=|v(m) \cdot \tau|=\left|\frac{x^{2}-y^{2}}{\sqrt{\left(2 x z+4 x^{3}\right)^{2}+\left(-2 y z+4 y^{3}\right)^{2}+\left(x^{2}-y^{2}\right)^{2}}}\right|$.

Agora, consideremos a curva $\gamma(t)=\left(0, t, t^{2}\right) \in V_{1}$. Então, em $(\star)$ obtemos:

$\left|\frac{\left(0^{2}-t^{2}\right)}{\left[\left(2.0 t^{2}+4.0^{3}\right)^{2}+\left(-2 t t^{2}+4 t^{3}\right)^{2}+\left(0^{2}-t^{2}\right)^{2}\right]^{\frac{1}{2}}}\right|=\left|\frac{t^{2}}{\left[t^{4}\left(4 t^{2}+1\right)\right]^{\frac{1}{2}}}\right| \nrightarrow 0$ quando $t \rightarrow 0$.

Portanto, $\lim _{m \rightarrow p}\left|\pi_{N_{m} X}(\tau)\right| \neq 0$. Logo, esta estratificação não é $(a)$-regular.

(ii) Estratificação de $V_{1}$ em Estrat.2:

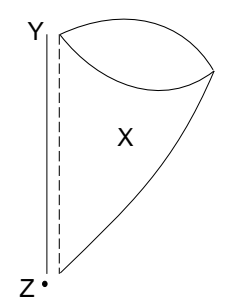

Figura 1.14:

Aqui, temos os seguintes casos: 
Caso I: $Y$ é $(a)$-regular sobre $Z$ ?

Sim, este é um caso trivial visto que o estrato $Z$ é apenas um ponto.

Caso II: $X$ é $(a)$-regular sobre $Z$ ?

Sim, análogo ao Caso I.

Caso III: $X$ é $(a)$-regular sobre $Y$ para algum ponto $p \in Y$ ?

Sim, considere a seguinte figura:

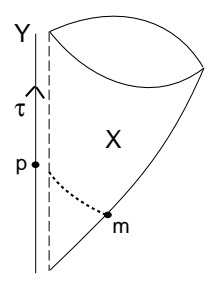

Figura 1.15:

Seja $\{m\}$ uma seqüência de pontos em $X$ tal que $m \rightarrow p \in Y$ (como indicado na linha pontilhada da figura acima). O espaço tangente $T_{m} X$ é um espaço linear 2-dimensional; logo, é claro que $\tau \in T_{p} Y$ está contido em $T_{m} X$, quando $m \rightarrow p$.

Além disso, os 3 casos esgotam todas as possibilidades em nosso processo de checagem, portanto esta estratificação satisfaz a condição de regularidade $(a)$, ou seja, a estratificação é (a)-regular.

No exemplo 1.9, temos que a variedade algébrica $V_{2}$ é definida por $V_{2}=\left\{(x, y) \in \mathbb{R}^{2} ; f_{2}(x, y)=\right.$ $0\}$, onde $f_{2}(x, y)=x\left(x-y^{2}\right)$; então temos as seguintes situações:

(i) Estratificação de $V_{2}$ em Estrat.2:

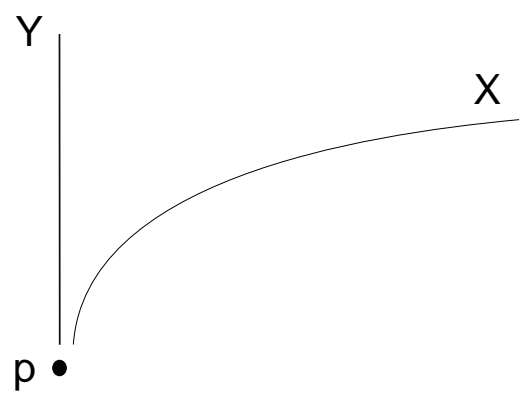

Figura 1.16:

Note que podemos dividir a figura acima em dois casos, a saber: 
Caso I:

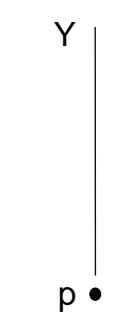

Figura 1.17:

\section{Caso II:}

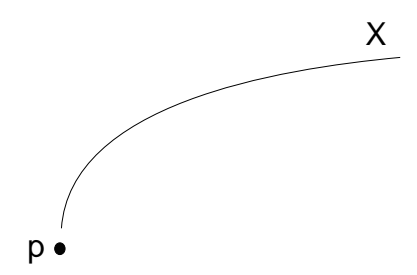

Figura 1.18:

É claro que $Y$ é $(a)$-regular sobre $p$ e que $X$ é $(a)$-regular sobre $p$, pois $p$ é apenas um ponto. Portanto, esta estratificação é $(a)$-regular.

(ii) Estratificação de $V_{2}$ em Estrat.1:

Considere a figura abaixo:

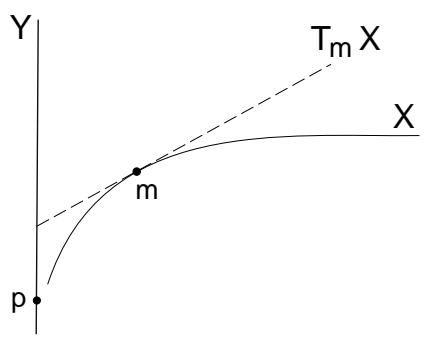

Figura 1.19:

Aqui, infelizmente, a estratificação é ( $a$ )-regular, visto que $\tau \in T_{p} Y$ está claramente contido em $T_{m} X$ quando $m \rightarrow p$. Dizemos "infelizmente", porque $p \in Y$ é, obviamente, "pior" que os outros pontos do estrato $Y$.

Finalmente, no exemplo 1.11, temos que a variedade $V_{4}=\left\{(x, y, z) \in \mathbb{R}^{n} ; f_{4}(x, y, z)=0\right\}$, onde $f_{4}(x, y, z)=y^{2}-z^{2} x^{2}-x^{3}$. Por um cálculo simples, podemos verificar que as duas estratificações de $V_{4}$ dadas na Figura1.11 e Figura1.12 são $(a)$-regulares. Mas, a origem no estrato 1 da Figura1.11 é, claramente, muito "pior" que os demais pontos deste mesmo estrato.

Portanto, precisamos de condições adicionais de regularidade. 


\section{Capítulo 2}

\section{Teoria de estratificação de Thom-Whitney}

Neste capítulo, introduziremos os principais resultados da teoria de estratificação de ThomWhitney que serão usados ao longo da dissertação, dentre eles, o resultado que garante a trivialidade topológica local de uma estratificação de Whitney. Em alguns destes resultados, serão feitas apenas observações, maiores detalhes podem ser encontrados em [13],[15] e [28].

\subsection{A (b)-regularidade de H. Whitney}

Antes de definirmos as condições de regularidade $(b)$ e $\left(b^{\prime}\right)$, fixemos as seguintes notações:

Sejam $X, Y$ subvariedades regulares sem bordo do $\mathbb{R}^{n}$.

(i) Dados $p \in Y$ e $m \in X$, denotaremos a projeção ortogonal do vetor $(\overrightarrow{m p})$ sobre o espaço tangente a $Y$ no ponto $p, \pi_{T_{p} Y}(\overrightarrow{m p})$, por $\pi_{p}(\overrightarrow{m p})$;

(ii) Considerando $\left\{m_{i}\right\}$ e $\left\{p_{i}\right\}$ seqüências de pontos em $X$ e $Y$, respectivamente, denotemos $\pi_{T_{p_{i}} Y}\left(\overrightarrow{m_{i} p_{i}}\right)$ por $\pi_{p_{i}}\left(\overrightarrow{m_{i} p_{i}}\right)$.

Definição 2.1. Dizemos que $X$ é $\left(b^{\prime}\right)$-regular sobre $Y$ no ponto $p \in Y$ se

$$
\lim _{m \rightarrow p} \pi_{N_{m} X}\left(\frac{\overrightarrow{m p}-\pi_{p}(\overrightarrow{m p})}{\left|\overrightarrow{m p}-\pi_{p}(\overrightarrow{m p})\right|}\right)=0,
$$

onde |.| é a norma euclideana e $N_{m} X$ é o espaço normal a $X$ em $M$.

Isto significa que a direção $\overrightarrow{m p}-\pi_{p}(\overrightarrow{m p})$ é paralela a $T_{m} X$.

Definição 2.2. Dizemos que $X$ é (b)-regular sobre $Y$ no ponto $p \in Y$ se

$$
\lim _{i \rightarrow \infty} \pi_{N_{m_{i}} X}\left(\frac{\overrightarrow{m_{i} p_{i}}-\pi_{p_{i}}\left(\overrightarrow{m_{i} p_{i}}\right)}{\left|\overrightarrow{m_{i} p_{i}}-\pi_{p_{i}}\left(\overrightarrow{m_{i} p_{i}}\right)\right|}\right)=0
$$

onde |.| é a norma euclideana. 
Ou seja, o par $(X, Y)$ satisfaz a condição $(b)$ em $p$, se:

Sendo $\left\{m_{i}\right\}$ uma seqüência de pontos em $X$ com $m_{i} \rightarrow p$ e $\left\{p_{i}\right\}$ uma seqüência de pontos em $Y$, também convergindo para $p$. Suponha que $T_{m_{i}} X$ convirja para algum plano $\tau \subset \mathbb{R}^{n}$, que $m_{i} \neq p_{i}$ para todo $i$ e que as secantes $\widehat{m_{i} p_{i}}$ convirjam (no espaço projetivo $\mathbb{P}^{n-1}$ ) para alguma reta $l \in \mathbb{R}^{n}$. Então, $l \subset \tau$.

Definição 2.3. Dizemos que $(X, Y)$ é (b)-regular se ele satisfaz a condição (b) em todo ponto de $Y$. Neste caso, diremos que o par $(X, Y)$ é Whitney regular ou simplesmente regular.

Seja $\left(X^{\prime}, Y^{\prime}\right)$ um segundo par de subvariedades regulares do $\mathbb{R}^{n}$, e seja $p^{\prime} \in Y^{\prime}$.

Lema 2.1. Suponha que existem vizinhanças abertas $U$ e $U^{\prime}$ de $p$ e $p^{\prime}$ no $\mathbb{R}^{n}$ e um difeomorfismo (suave) $\varphi: U \longrightarrow U^{\prime}$ tal que $\varphi(U \cap X)=U^{\prime} \cap X^{\prime}, \varphi(U \cap Y)=U^{\prime} \cap Y^{\prime}$ e $\varphi(p)=p^{\prime}$. Então $(X, Y)$ satisfaz a condição $(b)$ em $p$ se, e somente se, $\left(X^{\prime}, Y^{\prime}\right)$ satisfaz a condição $(b)$ em $p^{\prime}$.

Definição 2.4. Sejam $M$ uma variedade regular, e $X, Y$ subvariedades regulares. Seja $p \in Y$. Dizemos que $(X, Y)$ satisfaz a condição $(b)$ em p se para alguma carta $(U, \varphi)$ sobre $p$, temos que o par $(\varphi(U \cap X), \varphi(U \cap Y))$ satisfaz a condição $(b)$ em $\varphi(p)$.

Pelo Lema 2.1, se $(X, Y)$ satisfaz a condição $(b)$ em $p$; então, para toda carta $(U, \varphi)$ sobre $p$, temos que $(\varphi(U \cap X), \varphi(U \cap Y))$ satisfaz a condição $(b)$ em $p$.

\subsection{Resultados e Exemplos}

Nesta seção, provaremos que as condições de regularidade $(a)$ e $(b)$ são equivalentes às condições de regularidade $(a)$ e $\left(b^{\prime}\right)$. Veremos também que $(b)$ implica $(a)$; assim, para obtermos uma estratificação regular será suficiente checarmos a condição (b). Como em geral as condições $(a)$ e $\left(b^{\prime}\right)$ são mais fáceis de serem manuseadas que a condição $(b)$, principalmente quando o estrato de menor dimensão é 1-dimensional, em geral, estaremos trabalhando com as condições $(a)$ e $\left(b^{\prime}\right)$.

Teorema 2.1. As condições de regularidade $(a)$ e $(b)$ são equivalentes às condições $(a)$ e $\left(b^{\prime}\right)$.

Antes de provarmos o teorema acima apresentaremos alguns resultados e definições.

Definição 2.5. Seja $(X, Y)$ um par de estratos de alguma estratificação de uma variedade $V$, com $Y \subset \bar{X}$. Dizemos que o par $(X, Y)$ é Whitney regular, ou simplesmente regular, se a condição (b) (ou $(a)$ e $\left.\left(b^{\prime}\right)\right)$ for(em) satisfeita(s) em todo ponto de $Y$.

Definição 2.6. Uma estratificação de uma variedade $V$ é dita regular se, para todo par de estratos $(X, Y)$ de $V$, o par $(X, Y)$ é Whitney regular.

Exemplo 2.1. Seja $f_{5}(x, y, z)=y^{2}-z^{2} x^{3}-x^{5}$; então a variedade algébrica, definida por $f_{5}$, $V_{5}=\left\{(x, y, z) \in \mathbb{R}^{3} ; f_{5}(x, y, z)=0\right\}$ admite uma estratificação Whitney regular. 


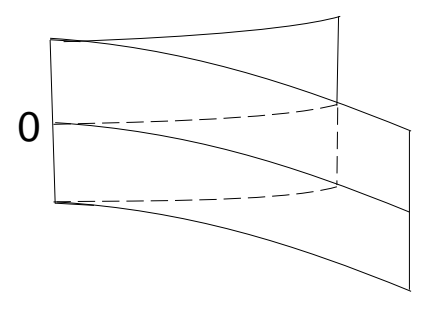

Figura 2.1: $V_{5}$

De fato, considere em $V_{5}$ a estratificação dada por: $Y=$ eixo $z$ e $X$ o complementar de $Y$ em $V_{5}$. Afirmamos que esta estratificação é regular. Com efeito, para todo $m=(x, y, z) \in X$, com $(x, y) \neq(0,0)$ temos que o espaço normal é gerado por:

$$
\nabla f_{5}(x, y, z)=\left(-3 x^{2} z^{2}-5 x^{4}, 2 y,-2 z x^{3}\right) .
$$

O vetor normal (direção) é:

$$
v(m)=\frac{\nabla f_{5}(x, y, z)}{\left\|\nabla f_{5}(x, y, z)\right\|}=\left(\frac{-3 x^{2} z^{2}-5 x^{4}}{\sqrt{A_{5}}}, \frac{2 y}{\sqrt{A_{5}}}, \frac{-2 z x^{3}}{\sqrt{A_{5}}}\right),
$$

onde: $A_{5}=\left(3 x^{2} z^{2}+5 x^{4}\right)^{2}+4 y^{2}+4 z^{2} x^{6}$.

Seja $p=\left(0,0, z_{0}\right)$. Como $T_{p} Y$ é 1-dimensional e $\tau \in T_{p} Y$, podemos assumir que $\tau=(0,0,1)$. Logo,

$$
\begin{aligned}
\left|\pi_{N_{m} X}(\tau)\right| & =|v(m) \cdot \tau| \\
& =\left|\left(\frac{-3 x^{2} z^{2}-5 x^{4}}{\sqrt{A_{5}}}, \frac{2 y}{\sqrt{A_{5}}}, \frac{-2 z x^{3}}{\sqrt{A_{5}}}\right) \cdot(0,0,1)\right| \\
& =\left|\frac{-2 z x^{3}}{\sqrt{\left(3 x^{2} z^{2}+5 x^{4}\right)^{2}+4 y^{2}+4 z^{2} x^{6}}}\right| .
\end{aligned}
$$

Elevando ambos os membros ao quadrado, temos:

$$
\begin{aligned}
\left|\pi_{N_{m} X}(\tau)\right|^{2} & =\left|\frac{4 z^{2} x^{6}}{\left(3 x^{2} z^{2}+5 x^{4}\right)^{2}+4 y^{2}+4 z^{2} x^{6}}\right| \\
& \leq\left|\frac{4 z^{2} x^{6}}{4 y^{2}}\right|=\left|\frac{z^{2} x^{6}}{x^{3}\left(z^{2}+x^{2}\right)}\right|=\left|\frac{z^{2} x^{3}}{z^{2}+x^{2}}\right| \longrightarrow 0 \text { quando } x \rightarrow 0 .
\end{aligned}
$$

Observe que em $V_{5}$ os pontos $(x, y, z)$ satisfazem a relação $y^{2}=x^{3}\left(z^{2}+x^{2}\right)$, assim $x \rightarrow 0$ se, e só se, $y \rightarrow 0$. Portanto, $\lim _{m \rightarrow p}\left|\pi_{N_{m} X}(\tau)\right|=0$ e $X$ é $(a)$-regular sobre $Y$.

Afirmamos que $X$ é $\left(b^{\prime}\right)$-regular sobre $Y$. De fato, temos que:

$$
\overrightarrow{m p}=\left(x, y, z-z_{0}\right) \Longrightarrow \pi_{p}(\overrightarrow{m p})=\left(0,0, z-z_{0}\right) \Longrightarrow \overrightarrow{m p}-\pi_{p}(\overrightarrow{m p})=(x, y, 0)
$$

Deste modo, segue-se que:

$$
\frac{\overrightarrow{m p}-\pi_{p}(\overrightarrow{m p})}{\left|\overrightarrow{m p}-\pi_{p}(\overrightarrow{m p})\right|}=\frac{(x, y, 0)}{\sqrt{x^{2}+y^{2}}}
$$


Então,

$$
\begin{aligned}
\left|\pi_{N_{m} X}\left(\frac{\overrightarrow{m p}-\pi_{p}(\overrightarrow{m p})}{\left|\overrightarrow{m p}-\pi_{p}(\overrightarrow{m p})\right|}\right)\right| & =\left|\left\langle v(m), \frac{(x, y, 0)}{\sqrt{x^{2}+y^{2}}}\right\rangle\right| \\
& =\left|\frac{-3 x^{3} z^{2}-5 x^{5}+2 y^{2}}{\sqrt{\left(3 x^{2} z^{2}+5 x^{4}\right)^{2}+4 y^{2}+4 z^{2} x^{6}} \sqrt{x^{2}+y^{2}}}\right|
\end{aligned}
$$

Elevando ambos os membros ao quadrado, temos:

$$
\begin{aligned}
\left|\pi_{N_{m} X}\left(\frac{\overrightarrow{m p}-\pi_{p}(\overrightarrow{m p})}{\left|\overrightarrow{m p}-\pi_{p}(\overrightarrow{m p})\right|}\right)\right|^{2} & =\left|\frac{\left(-3 x^{3} z^{2}-5 x^{5}+2 y^{2}\right)^{2}}{\left[\left(3 x^{2} z^{2}+5 x^{4}\right)^{2}+4 y^{2}+4 z^{2} x^{6}\right]\left(x^{2}+y^{2}\right)}\right| \\
& =\left|\frac{\left(-3 x^{3} z^{2}-5 x^{5}+2 z^{2} x^{3}+2 x^{5}\right)^{2}}{\left(x^{2}+y^{2}\right)\left[\left(3 x^{2} z^{2}+5 x^{4}\right)^{2}+4\left(z^{2} x^{3}+x^{5}\right)+4 z^{2} x^{6}\right]}\right| \\
& =\left|\frac{\left(x^{3} z^{2}+3 x^{5}\right)^{2}}{\left(x^{2}+y^{2}\right)\left[\left(3 x^{2} z^{2}+5 x^{4}\right)^{2}+4\left(z^{2} x^{3}+x^{5}\right)+4 z^{2} x^{6}\right]}\right| \\
& =\left|\frac{x^{6}\left(z^{2}+3 x^{2}\right)^{2}}{\left(x^{2}+y^{2}\right)\left[\left(3 x^{2} z^{2}+5 x^{4}\right)^{2}+4\left(z^{2} x^{3}+x^{5}\right)+4 z^{2} x^{6}\right]}\right| \\
& \leq\left|\frac{\left.x^{4}+3 x^{2}\right)^{2}}{\left(3 x^{2} z^{2}+5 x^{4}\right)^{2}+4\left(z^{2} x^{3}+x^{5}\right)+4 z^{2} x^{6}}\right| \\
& \leq\left|\frac{x^{4}\left(z^{2}+3 x^{2}\right)^{2}}{4\left(z^{2} x^{3}+x^{5}\right)}\right| \\
& =\left|\frac{x\left(z^{2}+3 x^{2}\right)^{2}}{4\left(z^{2}+x^{2}\right)}\right| \longrightarrow 0 \text { quando } x \rightarrow 0 .
\end{aligned}
$$

Portanto, $\lim _{m \rightarrow p}\left|\pi_{N_{m} X}\left(\frac{\overrightarrow{m p}-\pi_{p}(\overrightarrow{m p})}{\left|\overrightarrow{m p}-\pi_{p}(\overrightarrow{m p})\right|}\right)\right|=0$, ou seja, $X$ é $\left(b^{\prime}\right)$-regular sobre $Y$.

Consequentemente, $X$ é regular sobre $Y$.

Proposição 2.1. Se o par de estratos $(X, Y)$ satisfaz a condição $(b)$ em $p \in Y$, então também vale a condição $(a)$ em $p$.

Demonstração: Visto que as condições $(a)$ e $(b)$ são propriedades locais, podemos supor que $X$ e $Y$ são subvariedades regulares do $\mathbb{R}^{n}$. Seja $\left\{m_{i}\right\}$ uma seqüência de pontos em $X$ tal que $m_{i} \rightarrow p \in Y$ e que $T_{m_{i}} X$ converge para algum plano $\tau \subset T_{p} \mathbb{R}^{n} \cong \mathbb{R}^{n}$. Devemos mostrar que $T_{p} Y \subset \tau$.

Suponhamos que $T_{p} Y \not \subset \tau$. Então, existe uma reta $l \subset \mathbb{R}^{n}$, passando pela origem, tal que $l \subset T_{p} Y$, mas $l \not \subset \tau=\lim _{i \rightarrow \infty} T_{m_{i}} X$.

Como $l \subset T_{p} Y$, podemos escolher uma seqüência de pontos $\left\{p_{i}\right\} \subset Y$ tal que $p_{i} \neq m_{i}, p_{i} \rightarrow p$ e $\widehat{p_{i} m_{i}} \rightarrow l$. De fato, seja $m_{i}=\left(m_{i}^{1}, m_{i}^{2}, \ldots, m_{i}^{n}\right)$, como $m_{i} \rightarrow p$ seque que $m_{i}^{j} \rightarrow p$, para cada $1 \leq j \leq n$.

Agora, basta definirmos $p_{i}=\sqrt{\sup _{1 \leq j \leq n}\left\|m_{i}^{j}\right\|}\left(\frac{l}{\|l\|}\right)=\alpha_{i}\left(\frac{l}{\|l\|}\right) ;$ então: 


$$
\frac{m_{i}-p_{i}}{\left\|m_{i}-p_{i}\right\|}=\frac{m_{i}-\alpha_{i}\left(\frac{l}{\|l\|}\right)}{\left\|m_{i}-\alpha_{i}\left(\frac{l}{\|l\|}\right)\right\|}=\frac{\alpha_{i}\left(\frac{m_{i}}{\alpha_{i}}-\frac{l}{\|l\|}\right)}{\alpha_{i}\left\|\frac{m_{i}}{\alpha_{i}}-\frac{l}{\|l\|}\right\|} .
$$

Mas, para cada $1 \leq k \leq n$, temos que:

$$
0 \leq\left\|\frac{m_{i}^{k}}{\alpha_{i}}\right\|=\frac{\left\|m_{i}^{k}\right\|}{\sqrt{\sup _{1 \leq j \leq n}\left\|m_{i}^{j}\right\|}} \leq \frac{\sup _{1 \leq j \leq n}\left\|m_{i}^{j}\right\|}{\sqrt{\sup _{1 \leq j \leq n}\left\|m_{i}^{j}\right\|}}=\sqrt{\sup _{1 \leq j \leq n}\left\|m_{i}^{j}\right\|} \longrightarrow 0 \text { quando } i \rightarrow \infty .
$$

Logo, $\frac{m_{i}-p_{i}}{\left\|m_{i}-p_{i}\right\|} \longrightarrow \frac{-l}{\|l\|}$. Como $l \not \subset \tau$, isto contradiz a condição $(b)$.

Portanto, $T_{p} Y \subset \tau$, ou seja, $(X, Y)$ satisfaz a condição $(a)$ em $p$.

O próximo exemplo mostra que a condição $\left(b^{\prime}\right)$ não implica a condição $(a)$.

Exemplo 2.2. Lembre-se que a estratificação da variedade $V_{1}$ dada em Estrat.1 não satisfaz a condição de regularidade $(a)$. Afirmamos que ela é $\left(b^{\prime}\right)$-regular. De fato, já sabemos que o vetor normal (direção) é:

$$
v(m)=\frac{\nabla f_{1}(x, y, z)}{\left\|\nabla f_{1}(x, y, z)\right\|}=\left(\frac{2 x z+4 x^{3}}{\sqrt{A_{1}}}, \frac{-2 y z+4 y^{3}}{\sqrt{A_{1}}}, \frac{x^{2}-y^{2}}{\sqrt{A_{1}}}\right),
$$

onde $A_{1}=\left(2 x z+4 x^{3}\right)^{2}+\left(-2 y z+4 y^{3}\right)^{2}+\left(x^{2}-y^{2}\right)^{2}$.

Seja $p=(0,0,0) \in Y$, então:

$$
\overrightarrow{m p}=\vec{m}=(x, y, z) \Longrightarrow \pi_{p}(\vec{m})=(0,0, z) \Longrightarrow \vec{m}-\pi_{p}(\vec{m})=(x, y, 0)
$$

Deste modo, segue-se que:

$$
\frac{\vec{m}-\pi_{p}(\vec{m})}{\left|\vec{m}-\pi_{p}(\vec{m})\right|}=\frac{(x, y, 0)}{|(x, y, 0)|}=\frac{(x, y, 0)}{\sqrt{x^{2}+y^{2}}} .
$$

Logo,

$$
\begin{aligned}
\left|\pi_{N_{m} X}\left(\frac{\vec{m}-\pi_{p}(\vec{m})}{\left|\vec{m}-\pi_{p}(\vec{m})\right|}\right)\right| & =\left|\left\langle v(m), \frac{(x, y, 0)}{\sqrt{x^{2}+y^{2}}}\right\rangle\right| \\
& =\left|\frac{2 x^{2} z+4 x^{4}-2 y^{2} z+4 y^{4}}{\sqrt{\left(2 x z+4 x^{3}\right)^{2}+\left(-2 y z+4 y^{3}\right)^{2}+\left(x^{2}-y^{2}\right)^{2}} \sqrt{x^{2}+y^{2}}}\right| \\
& =\left|\frac{2 x^{4}+2 y^{4}}{\left[\left(2 x z+4 x^{3}\right)^{2}+\left(-2 y z+4 y^{3}\right)^{2}+\left(x^{2}-y^{2}\right)^{2}\right]^{\frac{1}{2}}\left(x^{2}+y^{2}\right)^{\frac{1}{2}}}\right| .
\end{aligned}
$$

Afirmamos que $\lim _{m \rightarrow p}(*)=0$. De fato, pelo Lema de seleção da curva, obtemos uma curva analítica $a(s)=\left(x_{0} s^{n}+\ldots, y_{0} s^{m}+\ldots, z_{0} s^{p}+\ldots\right)$, com $a(0)=0$ e $a(s) \in V_{1}$ se $s \neq 0$. Vamos verificar se $\lim _{s \rightarrow 0}(*) \circ a(s)=0$. 
Primeiramente, note que

$$
\begin{aligned}
& z_{0} x_{0}^{2} s^{2 n+p}-z_{0} y_{0}^{2} s^{2 m+p}+x_{0}^{4} s^{4 n}+y_{0}^{4} s^{4 m}+\ldots=0 \Longleftrightarrow \\
& \Longleftrightarrow z_{0} x_{0}^{2} s^{2 n+p}-z_{0} y_{0}^{2} s^{2 m+p}+\ldots=-x_{0}^{4} s^{4 n}-y_{0}^{4} s^{4 m}+\ldots
\end{aligned}
$$

Além disso, considere:

$g\left(x_{0}, y_{0}, z_{0}\right)=\left(2 x_{0} z_{0} s^{n+p}+4 x_{0}^{3} s^{3 n}\right)^{2}+\left(-2 y_{0} z_{0} s^{m+p}+4 y_{0}^{3} s^{3 m}\right)^{2}+\left(x_{0}^{2} s^{2 n}-y_{0}^{2} s^{2 m}\right)^{2}+\ldots$

Basta considerarmos os seguintes casos:

Caso I: $n<m \Rightarrow 2 n+p=4 n \Leftrightarrow p=2 n$.

Em $(*)$, obtemos:

$$
\left|\frac{\left(2 x_{0}^{4} s^{4 n}+2 y_{0}^{4} s^{4 m}+\ldots\right)}{\left[g\left(x_{0}, y_{0}, z_{0}\right)\right]^{\frac{1}{2}}\left(x_{0}^{2} s^{2 n}+y_{0}^{2} s^{2 m}+\ldots\right)^{\frac{1}{2}}}\right| \sim \frac{s^{4 n}}{s^{2 n} s^{n}} \sim s^{n} \longrightarrow 0 \text { quando } s \rightarrow 0,
$$

onde $A \sim B$ significa que $A$ e $B$ possuem a mesma ordem.

Caso II: $n>m \Rightarrow 2 m+p=4 m \Leftrightarrow p=2 m$.

Em $(*)$, obtemos:

$$
\left|\frac{\left(2 x_{0}^{4} s^{4 n}+2 y_{0}^{4} s^{4 m}+\ldots\right)}{\left[g\left(x_{0}, y_{0}, z_{0}\right)\right]^{\frac{1}{2}}\left(x_{0}^{2} s^{2 n}+y_{0}^{2} s^{2 m}+\ldots\right)^{\frac{1}{2}}}\right| \sim \frac{s^{4 m}}{s^{2 m} s^{m}} \sim s^{m} \longrightarrow 0 \text { quando } s \rightarrow 0 .
$$

Caso III: $n=m$.

Em $(*)$, obtemos:

$\left|\frac{\left(2 x_{0}^{4}+2 y_{0}^{4}+\ldots\right) s^{4 n}}{\left[\left(2 x_{0} z_{0} s^{n+p}+4 x_{0}^{3} s^{3 n}\right)^{2}+\left(-2 y_{0} z_{0} s^{n+p}+4 y_{0}^{3} s^{3 n}\right)^{2}+\left(x_{0}-y_{0}\right)^{2} s^{4 n}\right]^{\frac{1}{2}}\left[\left(x_{0}+y_{0}\right)^{2} s^{2 n}\right]^{\frac{1}{2}}+\ldots}\right|$.

Aqui, temos duas possibilidades:

(i) Se $\left|x_{0}\right| \neq\left|y_{0}\right|$, então $p=2 n$; logo

$$
\begin{aligned}
& \left|\frac{\left(2 x_{0}^{4}+2 y_{0}^{4}+\ldots\right) s^{4 n}}{\left[\left(2 x_{0} z_{0} s^{n+p}+4 x_{0}^{3} s^{3 n}\right)^{2}+\left(-2 y_{0} z_{0} s^{n+p}+4 y_{0}^{3} s^{3 n}\right)^{2}+\left(x_{0}-y_{0}\right)^{2} s^{4 n}\right]^{\frac{1}{2}}\left[\left(x_{0}+y_{0}\right)^{2} s^{2 n}\right]^{\frac{1}{2}}+\ldots}\right| \sim \\
& \quad \sim \frac{s^{4 n}}{s^{2 n} s^{n}} \sim s^{n} \longrightarrow 0 \text { quando } s \rightarrow 0 .
\end{aligned}
$$

(ii) Se $\left|x_{0}\right|=\left|y_{0}\right|$, então $p<2 n$; logo

$$
\begin{aligned}
& \left|\frac{\left(2 x_{0}^{4}+2 y_{0}^{4}+\ldots\right) s^{4 n}}{\left[\left(2 x_{0} z_{0} s^{n+p}+4 x_{0}^{3} s^{3 n}\right)^{2}+\left(-2 y_{0} z_{0} s^{n+p}+4 y_{0}^{3} s^{3 n}\right)^{2}+\left(x_{0}-y_{0}\right)^{2} s^{4 n}\right]^{\frac{1}{2}}\left[\left(x_{0}+y_{0}\right)^{2} s^{2 n}\right]^{\frac{1}{2}}+\ldots}\right| \sim \\
& \quad \sim \frac{s^{4 n}}{s^{\frac{2 n+p}{2}} s^{n}} \sim \frac{s^{2 n}}{s^{\frac{p}{2}}} \longrightarrow 0 \text { quando } s \rightarrow 0 \text {, pois } p<2 n .
\end{aligned}
$$

Portanto, esta estratificação é ( $\left.b^{\prime}\right)$-regular. 
Exemplo 2.3. Outro exemplo que satisfaz a condição de regularidade $\left(b^{\prime}\right)$ mas não satisfaz a condição (a) é o "Guarda-chuva de Whitney" com a estratificação dada na Figura 1.9, ou seja, para $Y=\left\{(0,0, z) \in \mathbb{R}^{3} ; z \geq 0\right\}$ e $X=f_{3}^{-1}(0) \backslash Y$.

Lembre-se que $f_{3}(x, y, z)=x^{2}-y^{2} z$. Para todo $m=(x, y, z) \in X,(x, y) \neq(0,0)$, temos que o espaço normal é gerado por:

$$
\nabla f_{3}(x, y, z)=\left(2 x,-2 y z,-y^{2}\right)
$$

O vetor normal (direção) é:

$$
v(m)=\frac{\nabla f_{3}(x, y, z)}{\left\|\nabla f_{3}(x, y, z)\right\|}=\left(\frac{2 x}{\sqrt{A_{3}}}, \frac{-2 y z}{\sqrt{A_{3}}}, \frac{-y^{2}}{\sqrt{A_{3}}}\right), \text { onde } A_{3}=\sqrt{4 x^{2}+4 y^{2} z^{2}+y^{4}} .
$$

Afirmação 1: Esta estratificação não é $(a)$-regular. De fato, seja $p=(0,0,0) \in Y$. Note que, se $\tau \in T_{0} Y$, podemos assumir que $\tau=(0,0,1)$, visto que $T_{0} Y$ é 1-dimensional. Logo,

$$
\left|\pi_{N_{m} X}(\tau)\right|=|v(m) \cdot \tau|=\left|\frac{-y^{2}}{\sqrt{4 x^{2}+4 y^{2} z^{2}+y^{4}}}\right| .
$$

Elevando ao quadrado, temos:

$$
\begin{aligned}
\left|\pi_{N_{m} X}(\tau)\right|^{2} & =\left|\frac{y^{4}}{4 x^{2}+4 y^{2} z^{2}+y^{4}}\right| \\
& =\left|\frac{y^{4}}{4 y^{2} z+4 y^{2} z^{2}+y^{4}}\right| \\
& =\left|\frac{y^{2}}{4 z+4 z^{2}+y^{2}}\right| \\
& =\left|\frac{1}{\frac{4 z+4 z^{2}}{y^{2}}+1}\right| \nrightarrow 0 \text { quando } m \rightarrow p .
\end{aligned}
$$

Portanto, $\lim _{m \rightarrow p}\left|\pi_{N_{m} X}(\tau)\right| \neq 0$, ou seja, esta estratificação não é $(a)$-regular.

Afirmação 2: Esta estratificação é ( $\left.b^{\prime}\right)$-regular. De fato, temos que:

$$
\overrightarrow{m p}=\vec{m}=(x, y, z) \Longrightarrow \pi_{p}(\vec{m})=(0,0, z) \Longrightarrow \vec{m}-\pi_{p}(\vec{m})=(x, y, 0) .
$$

Deste modo, segue-se que:

$$
\frac{\vec{m}-\pi_{p}(\vec{m})}{\left|\vec{m}-\pi_{p}(\vec{m})\right|}=\frac{(x, y, 0)}{\sqrt{x^{2}+y^{2}}}
$$

Logo,

$$
\begin{aligned}
\left|\pi_{N_{m} X}\left(\frac{\vec{m}-\pi_{p}(\vec{m})}{\left|\vec{m}-\pi_{p}(\vec{m})\right|}\right)\right| & =\left|\left\langle v(m), \frac{(x, y, 0)}{\sqrt{x^{2}+y^{2}}}\right\rangle\right| \\
& =\left|\frac{2 x^{2}-2 y^{2} z}{\sqrt{4 x^{2}+4 y^{2} z^{2}+y^{4}} \sqrt{x^{2}+y^{2}}}\right|
\end{aligned}
$$




$$
=\left|\frac{2 y^{2} z-2 y^{2} z}{\left(4 x^{2}+4 y^{2} z^{2}+y^{4}\right)^{\frac{1}{2}}\left(x^{2}+y^{2}\right)^{\frac{1}{2}}}\right|=0 .
$$

Assim, $\lim _{m \rightarrow p} \pi_{N_{m} X}\left(\frac{\vec{m}-\pi_{p}(\vec{m})}{\left|\vec{m}-\pi_{p}(\vec{m})\right|}\right)=0$ e $(X, Y)$ é $\left(b^{\prime}\right)$-regular.

O próximo exemplo mostra que a condição de regularidade $(a)$ não implica a condição de regularidade $\left(b^{\prime}\right)$.

Exemplo 2.4. ("Cúspide de Whitney") Lembre-se que $f_{4}(x, y, z)=y^{2}-z^{2} x^{2}-x^{3}$ e que $V_{4}=$ $\left\{(x, y, z) \in \mathbb{R}^{3} ; f_{4}(x, y, z)=0\right\}$. Sejam $Y=$ eixo $z$ e $X=f_{4}^{-1}(0) \backslash Y$.

Afirmamos que o par de estratos $(X, Y)$ é $(a)$-regular. De fato, para todo $m=(x, y, z) \in X$, com $(x, y) \neq(0,0)$, o espaço normal é gerado por:

$$
\nabla f_{4}(x, y, z)=\left(-2 x z^{2}-3 x^{2}, 2 y,-2 x^{2} z\right)
$$

E, o vetor normal (direção) é:

$$
v(m)=\frac{\nabla f_{4}(x, y, z)}{\left|\nabla f_{4}(x, y, z)\right|}=\left(\frac{-2 x z^{2}-3 x^{2}}{\sqrt{A_{4}}}, \frac{2 y}{\sqrt{A_{4}}}, \frac{-2 x^{2} z}{\sqrt{A_{4}}}\right),
$$

onde $A_{4}=\left(2 x z^{2}+3 x^{2}\right)^{2}+4 y^{2}+4 x^{4} z^{2}$.

Sejam $p=(0,0,0) \in Y$ e $\tau \in T_{p} Y$ mas, como $T_{p} Y$ é 1-dimensional, podemos assumir que $\tau=(0,0,1)$. Então,

$$
\left|\pi_{N_{m} X}(\tau)\right|=|v(m) \cdot \tau|=\left|\frac{4 x^{4} z^{2}}{\sqrt{\left(2 x z^{2}+3 x^{2}\right)^{2}+4 y^{2}+4 x^{4} z^{2}}}\right| .
$$

Elevando ambos os lados ao quadrado, temos que:

$$
\left|\pi_{N_{m} X}(\tau)\right|^{2}=\left|\frac{16 x^{8} z^{4}}{\left(2 x z^{2}+3 x^{2}\right)^{2}+4 y^{2}+4 x^{4} z^{2}}\right| \leq\left|\frac{16 x^{8} z^{4}}{4 y^{2}}\right|=\left|\frac{4 x^{8} z^{4}}{y^{2}}\right|
$$

Observe que em $V_{4}$ os pontos $(x, y, z)$ satisfazem a relação $y^{2}=x^{2}\left(z^{2}+x\right)$, assim $x \rightarrow 0$ se, e só se, $y \rightarrow 0$. Logo,

$$
\left|\pi_{N_{m} X}(\tau)\right|^{2} \leq\left|\frac{4 x^{8} z^{4}}{y^{2}}\right|=\left|\frac{4 x^{8} z^{4}}{x^{2}\left(z^{2}+x\right)}\right|=\left|\frac{4 x^{6} z^{4}}{z^{2}+x}\right| \longrightarrow 0 \text { quando } x \rightarrow 0 .
$$

Portanto, $\lim _{m \rightarrow p}\left|\pi_{N_{m} X}(\tau)\right|=0$, ou seja, $(X, Y)$ é $(a)$-regular em $(0,0,0)$.

Agora, afirmamos que $\left(b^{\prime}\right)$ falha ao longo da $\gamma(s)=\left(-s^{2}, 0, s\right) \in V_{4}$. De fato,

$$
\overrightarrow{m p}=\vec{m}=(x, y, z) \Longrightarrow \pi_{p}(\overrightarrow{m p})=(0,0, z) \Longrightarrow \overrightarrow{m p}-\pi_{p}(\overrightarrow{m p})=(x, y, 0)
$$

Deste modo, segue-se que:

$$
\frac{\overrightarrow{m p}-\pi_{p}(\overrightarrow{m p})}{\left|\overrightarrow{m p}-\pi_{p}(\overrightarrow{m p})\right|}=\frac{(x, y, 0)}{\sqrt{x^{2}+y^{2}}}
$$


Assim, obtemos

$$
\begin{aligned}
\left|\pi_{N_{m} X}\left(\frac{\overrightarrow{m p}-\pi_{p}(\overrightarrow{m p})}{\left|\overrightarrow{m p}-\pi_{p}(\overrightarrow{m p})\right|}\right)\right| & =\left|\left\langle v(m), \frac{(x, y, 0)}{\sqrt{x^{2}+y^{2}}}\right\rangle\right| \\
& =\left|\frac{-2 x^{2} z^{2}-3 x^{3}+2 y^{2}}{\sqrt{\left(2 x z^{2}+3 x^{2}\right)^{2}+4 y^{2}+4 x^{4} z^{2}} \sqrt{x^{2}+y^{2}}}\right| .
\end{aligned}
$$

Em $\gamma(s)$ isto se torna:

$$
\begin{aligned}
\left|\left\langle v(\gamma(s)), \frac{\left(-s^{2}, 0,0\right)}{\sqrt{\left(-s^{2}\right)^{2}+0}}\right\rangle\right| & =\left|\frac{-2\left(-s^{2}\right)^{2} s^{2}-3\left(-s^{2}\right)^{3}+2.0}{\sqrt{\left[2\left(-s^{2}\right) s^{2}+3\left(-s^{2}\right)^{2}\right]^{2}+4.0+4\left(-s^{2}\right)^{4} s^{2}} \sqrt{\left(-s^{2}\right)^{2}+0}}\right| \\
& =\left|\frac{-2 s^{4} \cdot s^{2}+3 s^{6}}{\sqrt{\left(-2 s^{4}+3 s^{4}\right)^{2}+4 s^{10} \sqrt{s^{4}}}}\right| \\
& =\left|\frac{-2 s^{6}+3 s^{6}}{\sqrt{\left(s^{4}\right)^{2}+4 s^{10}} s^{2}}\right| \\
& =\left|\frac{s^{6}}{\sqrt{s^{8}\left(1+4 s^{2}\right)} s^{2}}\right| \\
& =\left|\frac{s^{4}}{s^{4} \sqrt{1+4 s^{2}}}\right|=\left|\frac{1}{\sqrt{1+4 s^{2}}}\right| \nrightarrow 0 \text { quando } s \rightarrow 0 .
\end{aligned}
$$

Portanto, esta estratificação não é ( $\left.b^{\prime}\right)$-regular em $(0,0,0)$.

Observação 2.1. É fácil verificar que a estratificação dada no exemplo acima satisfaz as condições $(a)$ e $\left(b^{\prime}\right)$ fora da origem, ou seja, exceto na origem esta é uma estratificação de Whitney. Mais ainda, $p=(0,0,0)$ é o ponto "ruim" desta estratificação.

Lembre-se que nossa intenção em adicionar condições de regularidade era para distinguir a origem no estrato $Y$ de $V_{2}=\left\{(x, y) \in \mathbb{R}^{2} ; f_{2}(x, y)=0\right\}$, onde $f_{2}(x, y)=x\left(x-y^{2}\right)$, em Estra.1. Então, vamos trabalhar neste exemplo:

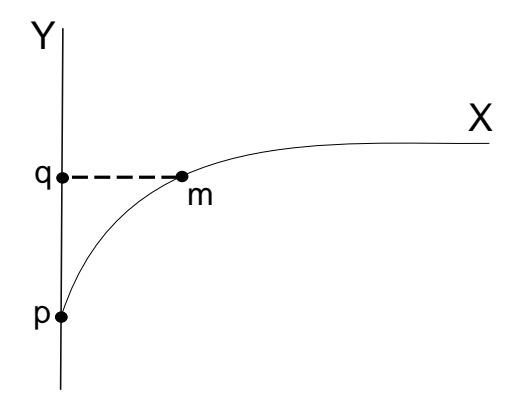

Figura 2.2:

Afirmamos que a estratificação dada na figura acima não é regular. De fato, pois para todo $m=(x, y) \in X$ temos que o espaço normal é gerado por:

$$
\nabla f_{2}(x, y)=\left(2 x-y^{2},-2 x y\right)
$$


O vetor normal (direção) é:

$$
v(m)=\frac{\nabla f_{2}(x, y)}{\left\|\nabla f_{2}(x, y)\right\|}=\left(\frac{2 x-y^{2}}{\sqrt{\left(2 x-y^{2}\right)^{2}+4 x^{2} y^{2}}}, \frac{-2 x y}{\sqrt{\left(2 x-y^{2}\right)^{2}+4 x^{2} y^{2}}}\right) .
$$

Como $m \in X$, podemos escrever $m=\left(y^{2}, y\right)$. Seja $p=(0,0) \in Y$. Então:

$$
\overrightarrow{m p}=\vec{m}=\left(y^{2}, y\right) \Longrightarrow \pi_{p}(\vec{m})=(0, y) \Longrightarrow \vec{m}-\pi_{p}(\vec{m})=\left(y^{2}, 0\right)
$$

E, consequentemente, temos que:

$$
\frac{\vec{m}-\pi_{p}(\vec{m})}{\left|\vec{m}-\pi_{p}(\vec{m})\right|}=\frac{\left(y^{2}, 0\right)}{\sqrt{y^{4}}}=\frac{\left(y^{2}, 0\right)}{y^{2}}=(1,0)
$$

Logo,

$$
\begin{aligned}
\left|\pi_{N_{m} X}\left(\frac{\vec{m}-\pi_{p}(\vec{m})}{\left|\vec{m}-\pi_{p}(\vec{m})\right|}\right)\right| & =\left|\frac{2 x-y^{2}}{\sqrt{\left(2 x-y^{2}\right)^{2}+4 x^{2} y^{2}}}\right| \\
& =\left|\frac{2 y^{2}-y^{2}}{\left[\left(2 y^{2}-y^{2}\right)^{2}+4 y^{4} y^{2}\right]^{\frac{1}{2}}}\right| \\
& =\left|\frac{y^{2}}{\left(y^{4}+4 y^{6}\right)^{\frac{1}{2}}}\right| \\
& =\left|\frac{1}{\left(1+4 y^{2}\right)^{\frac{1}{2}}}\right| \nrightarrow 0 \text { quando } m \rightarrow p, \text { o que contradiz }\left(b^{\prime}\right) .
\end{aligned}
$$

Portanto, a estratificação de $V_{2}$ em Estrat.1 não é Whitney regular.

Observação 2.2. Depois da discussão destes exemplos é razoável esperar que se $V$ é estratificada por $X_{1} \cup \ldots \cup X_{p}$ tal que $X_{j}$ é $(b)$-regular sobre $X_{i}$ e $X_{i} \cap \overline{X_{j}} \neq \emptyset$ então:

$$
\operatorname{dim} X_{i}<\operatorname{dim} X_{j}
$$

Em geral, não é verdade que, se $X_{i} \cap \overline{X_{j}} \neq \emptyset$, então: $\operatorname{dim} X_{i}<\operatorname{dim} X_{j}$. Por exemplo, considere: $X_{1}=\{0\} \times[-1,1]$ em $\mathbb{R}^{2}$, e seja $X_{2}=\left\{(x, y) \in \mathbb{R}^{2} ; y=\sin \frac{1}{x}, x \neq 0\right\}$. Temos que, $X_{1} \cap \overline{X_{2}} \neq \emptyset$. Mas, $\operatorname{dim} X_{1}=\operatorname{dim} X_{2}$.

Observe a figura a seguir:

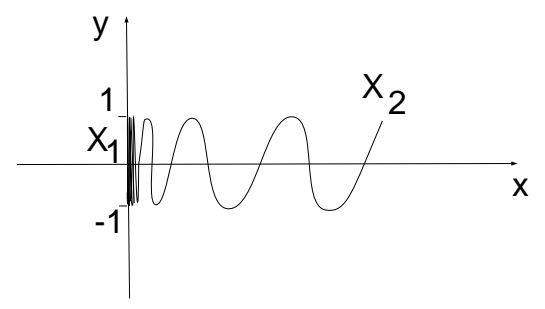

Figura 2.3: Seno topológico

Proposição 2.2. Suponha que $p \in \overline{X \backslash Y}$ e que $(X, Y)$ satisfaz a condição (b) em $p$. Então $\operatorname{dim} Y<\operatorname{dim} X$. 
Demonstração: Sendo o problema de caráter local, podemos assumir que $X=\mathbb{R}^{n}$. Como $p \in \overline{X \backslash Y}$, existe uma seqüência $\left\{m_{i}\right\} \subset X \backslash Y$ que converge para $p$. Pela compacidade da variedade Grasmanniana, podemos supor, passando para uma subseqüência, se necessário, que $T_{m_{i}} X$ converge para um $r$-plano $\tau \subset \mathbb{R}^{n}$ (onde $r=\operatorname{dim} X$ ).

Como $(b) \Rightarrow(a)$ (Proposição 2.1), $T_{p} Y \subset \tau$. Para $i$ suficientemente grande, existe um ponto $p_{i} \in Y$, que minimiza a distância para $m_{i}$. Novamente utilizando uma subseqüência, se necessário, podemos assumir que as secantes $\widehat{m_{i} p_{i}}$ convergem para uma reta $l \subset \mathbb{R}^{n}$. Como $p_{i}$ minimiza a distância para $m_{i}$, a secante $\widehat{m_{i} p_{i}}$ é ortogonal a $T_{p_{i}} Y$. Deste modo, segue que $l$ é ortogonal a $T_{p} Y$. Agora, como o par $(X, Y)$ satisfaz a condição $(b)$ em $p$, temos que $l \subset \tau$. Assim, mostramos que $T_{p} Y+l \subset \tau$ e $l$ é ortogonal a $T_{p} Y$.

Portanto, $\operatorname{dim} X=\operatorname{dim} \tau>\operatorname{dim} T_{p} Y=\operatorname{dim} Y$.

Definição 2.7. Dizemos que uma estratificação é localmente finita se o número de estratos é localmente finito.

Quando $V=X_{1} \cup \ldots \cup X_{p}$ é uma estratificação localmente finita tal que todos os pares de estratos adjacentes satisfazem a condição de fronteira e são (b)-regulares (ou $(a),\left(b^{\prime}\right)$-regulares) em todos os pontos, diremos que temos uma estratificação de Whitney de $V$.

Finalmente, provaremos o Teorema 2.1.

Demonstração: Afirmamos que $(a)+(b) \Leftrightarrow(a)+\left(b^{\prime}\right)$, isto é, $(b) \Leftrightarrow(a)+\left(b^{\prime}\right)$. De fato, note que é suficiente mostrarmos que $(a)+\left(b^{\prime}\right) \Rightarrow(b)$, pois a implicação contrária é óbvia.

Sejam $X, Y$ dois estratos tais que $Y \subset \bar{X}$. Seja $p \in Y$ um ponto arbitrário, então $X$ é (b)-regular sobre $Y$ em $p$, desde que $Y \cap X \neq \emptyset$. Com efeito, sejam $\left\{m_{i}\right\}$ e $\left\{p_{i}\right\}$ seqüências de pontos em $X$ e $Y$, respectivamente, tais que: $p_{i} \rightarrow p$ e $m_{i} \rightarrow p$, quando $i \rightarrow \infty$.

Observe que, próximo de $p, Y$ pode ser visto como $\mathbb{R}^{k} \times\{0\}$ e $X$ como $\mathbb{R}^{n}$. Seja $\pi$ a projeção de $X$ sobre $T_{p} Y$ numa vizinhança de $p$. Denote $\pi\left(m_{i}\right)$ por $q_{i}$, então $q_{i} \rightarrow p$, quando $i \rightarrow \infty$. Agora, considere os seguintes segmentos de reta:

$$
l_{i}^{(1)}=\overrightarrow{m_{i} q_{i}}, \quad l_{i}^{(2)}=\overrightarrow{q_{i} p_{i}} \quad \text { e } \quad l_{i}^{(3)}=\overrightarrow{m_{i} p_{i}},
$$

onde todos encontram-se num espaço euclideano n-dimensional $\left(\mathbb{R}^{n}\right)$. Então, podemos escrever:

$$
l_{i}^{(3)}=l_{i}^{(1)}+l_{i}^{(2)} .
$$

Note que a equação acima é uma equação de subespaços 1-dimensionais em $\mathbb{R}^{n}$.

Se os espaços $l_{i}^{(j)}, j=1,2,3$, forem transferidos para espaços lineares, levando os $q_{i}$ 's para a origem, então $(*)$ é uma relação precisa entre espaços lineares.

Agora, se $l_{i}^{(1)} \rightarrow l^{1}$ e $l_{i}^{(2)} \rightarrow l^{2}$, então $l_{i}^{(3)} \rightarrow l^{1}+l^{2}$.

Como $Y \subset \bar{X}$, se $T_{m_{i}} X \rightarrow \tau$, quando $i \rightarrow \infty$, então, pela condição de regularidade $\left(b^{\prime}\right)$, temos que $l^{1} \subset \tau$. Além disso, $l^{2} \subset T_{p} Y \cong \mathbb{R}^{k} \times\{0\}$. Logo, pela condição de regularidade $(a)$, $l^{2} \subset T_{p} Y \subset \tau$.

Deste modo, segue que $l^{1}+l^{2}=l^{3} \subset \tau$, e assim obtemos a condição de regularidade $(b)$. 


\subsection{Propriedades dos espaços Whitney estratificados}

Nesta seção, estaremos respondendo as seguintes questões:

(I) Dada uma variedade, ela admite uma estratificação de Whitney?

(II) Numa estratificação de Whitney, cada estrato contém pontos "igualmente ruins"?

(III) Como a estratificação de Whitney nos ajuda a estudar a estrutura local de uma aplicação suave? (Este é um dos objetivos fundamentais da teoria de singularidades de aplicações suaves).

Para responder (I), temos os seguintes teoremas:

Teorema 2.2. (Whitney, [28]): Toda variedade analítica (em $\mathbb{R}^{n}$ ou $\mathbb{C}^{n}$ ) admite, localmente, uma estratificação (b)-regular.

Teorema 2.3. (Lojasiewicz, [12]): Toda variedade semianalítica (em $\mathbb{R}^{n}$ ou $\mathbb{C}^{n}$ ) admite, localmente, uma estratificação (b)-regular.

Hironaka provou que o mesmo é verdade para conjuntos subanalíticos. Uma outra prova é dada por Denkowska, Wachta e Stasica em [9].

Para (II), temos:

Teorema 2.4. (Thom - Mather, [15] e [21]): Seja V estratificada sobre a condição de regularidade (b). Ao longo de cada estrato $X_{i}$, localmente, a figura topológica permanece invariante no seguinte sentido:

Se $x, y$ são dois pontos na componente conexa do estrato $X_{i}$, então existem uma vizinhança $U_{x}$ de $x$ em $V$, uma vizinhança, suficientemente pequena, $U_{y}$ de y em $V$, e um homeomorfismo $h$ tal que $h\left(X_{j}\right)=X_{j}$ e, além disso, $h\left(X_{j} \cap U_{x}\right)=h\left(X_{j} \cap U_{y}\right)$ para cada $j$.

Exemplo 2.5. Considere a figura abaixo:

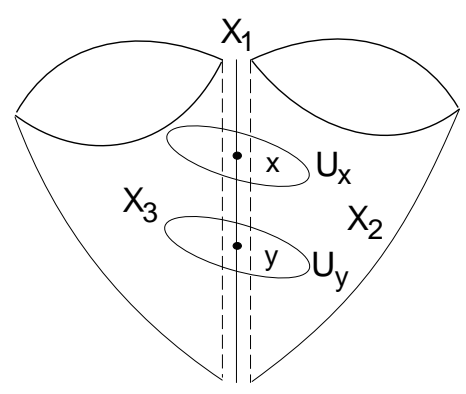

Figura 2.4:

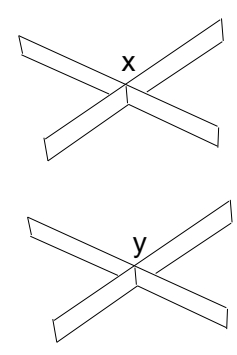

Figura 2.5:

Note que, localmente, em $x$ e $y$ temos que $U_{x} \cap X_{i}$ é homeomorfo a $U_{y} \cap X_{i}$, para cada $i=1,2,3$. 
Observação 2.3. O teorema 2.4 é importante porque, como temos dito, a idéia fundamental na noção de estratificação é decompor uma variedade numa união disjunta de estratos, onde cada estrato consiste de pontos igualmente "ruins". Este teorema justifica a afirmação que as condições de regularidade de Whitney satisfazem, intuitivamente, esta exigência.

Finalmente, para responder (III), temos:

Primeiramente, note que o problema mais geral da Análise diferencial, localmente, pode ser visto do seguinte modo:

Considere uma aplicação $G: U \longrightarrow \mathbb{R}^{p}$, de classe $C^{m}, G=\left(G_{1}, \ldots, G_{p}\right)$, onde $U \subset \mathbb{R}^{n}$ é aberto. Seja $A=\left\{x \in U ; G_{i}(x)=0, \forall i=1, \ldots, p\right\}$. O que podemos dizer sobre a estrutura topológica de $A$ ? Nos pontos em que $G$ tem posto máximo, o Teorema de Morse responde nossa questão no seguinte sentido:

Teorema 2.5. (Morse, [18]): Seja $M$ uma variedade diferenciável compacta, e seja $f: M \longrightarrow \mathbb{R}$ uma função diferenciável em $M$. Se $d f(p) \neq 0$, para todo $p \in M$, com $a \leq f(p) \leq b$, então $f^{-1}(a)$ é homeomorfo a $f^{-1}(b)$.

Observação 2.4. Os Lemas de Isotopia de Thom são generalizações do Teorema de Morse para variedades.

Agora, as aplicações em consideração serão todas diferenciáveis num conjunto aberto de um espaço euclideano contendo uma variedade $V$.

Teorema 2.6. (Primeiro Lema de Isotopia de Thom, [15] e [21]): Seja V uma variedade estratificada pela condição $(b)$ de Whitney. Seja $f: V \longrightarrow \mathbb{R}$ uma aplicação própria tal que, para cada estrato $X_{i}$ de $V$, a aplicação $\left.f\right|_{X_{i}}: X_{i} \longrightarrow(0,1)$ é uma submersão. Então, $f^{-1}(\alpha)$ é homeomorfo a $f^{-1}(\beta)$, para $\alpha, \beta \in(0,1)$.

Observação 2.5. A necessidade de $\left.f\right|_{X_{i}}$ ser uma submersão evita a seguinte situação:

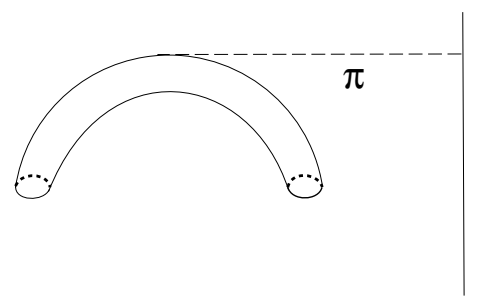

Figura 2.6:

Agora podemos perguntar: Sobre que condições duas aplicações terão o mesmo tipo topológico? Esta questão será respondida pelo Segundo Lema de Isotopia de Thom.

Antes de enunciarmos o Segundo Lema de Isotopia de Thom, consideremos as seguintes definições: 
Definição 2.8. Considerando o seguinte diagrama de espaços e aplicações,

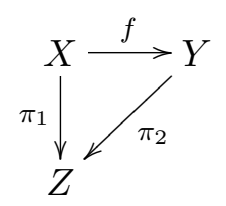

dizemos que a aplicação $f$ é trivial sobre $Z$, se existem espaços $X_{0}$ e $Y_{0}$, uma aplicação $f_{0}: X_{0} \longrightarrow Y_{0}$ e homeomorfismos $X \simeq X_{0} \times Z, Y \simeq Y_{0} \times Z$, de modo que o seguinte diagrama seja comutativo:

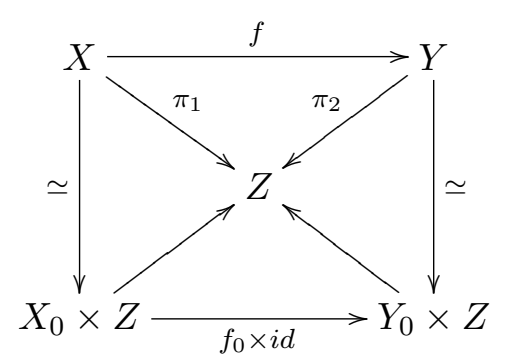

Definição 2.9. Dizemos que $f$ é localmente trivial sobre $Z$ se para todo $z \in Z$, existe uma vizinhança $U_{z}$ de $z$ em $Z$ tal que, no diagrama abaixo, $f$ é trivial sobre $U$ :

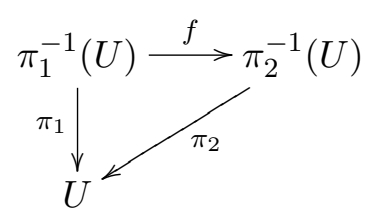

Observação 2.6. A trivialidade local de uma aplicação $f$ sobre um espaço $Z$ tem uma conseqüência que será muito importante no que segue:

Seja $\left\{f_{\alpha} ; \alpha \in Z\right\}$ uma família de aplicações, onde $f_{\alpha}: X_{\alpha} \longrightarrow Y_{\alpha}$ é a aplicação obtida pela restrição de $f$ à fibra $X_{\alpha}$ de $X$ sobre $\alpha$.

Se $f$ é localmente trivial sobre $Z$, então para quaisquer $\alpha, \beta \in Z$ as aplicações $f_{\alpha}$ e $f_{\beta}$ são equivalentes, no sentido que, existem homeomorfismos $h: X_{\alpha} \longrightarrow X_{\beta}$ e $k: Y_{\alpha} \longrightarrow Y_{\beta}$ tais que, $k \circ f_{\alpha}=f_{\beta} \circ h$.

Agora, suponhamos que $M, M^{\prime}$ e $P$ sejam variedades suaves e que $f: M \longrightarrow P$ seja $C^{\infty}$. Suponha, também, que $S \subset M$ e $S^{\prime} \subset M^{\prime}$ sejam variedades Whitney estratificadas.

Além disso, seja $g: M^{\prime} \longrightarrow M$ uma aplicação suave tal que $g\left(S^{\prime}\right) \subset S$. Então, o Segundo Lema de Isotopia de Thom nos dá condições suficientes para que o seguinte diagrama seja localmente trivial: 


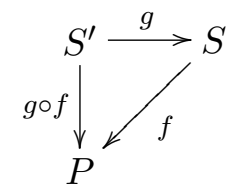

Definição 2.10. Sejam $X$ e $Y$ subvariedades regulares de $M^{\prime}$, e seja $p \in Y$. Suponha que $\left.g\right|_{X} e$ $\left.g\right|_{Y}$ tem posto constante. Dizemos que o par $(X, Y)$ satisfaz a condiçãa $\left(a_{g}\right)$ de Thom em $p$, se vale o seguinte:

Seja $\left\{m_{i}\right\}$ uma seqüência de pontos em $X$ tal que $m_{i} \rightarrow p$. Suponha que a seqüência de planos $\operatorname{ker}\left(\left.d g\right|_{X}\left(m_{i}\right)\right) \subset T_{m_{i}} M^{\prime}$ convirja para um plano $\tau \subset T_{p} Y$. Então, $\operatorname{ker}\left(\left.d g\right|_{Y}(p)\right) \subset \tau$.

Definição 2.11. Dizemos que o par $(X, Y)$ satisfaz a condição $\left(a_{g}\right)$ de Thom se ele satisfaz a condição $\left(a_{g}\right)$ para cada $p \in Y$.

Para que possamos entender melhor a condição $\left(a_{g}\right)$ de Thom, vamos trabalhar com o seguinte exemplo:

Sejam $M^{\prime}=$ cilíndro circular, $Y=$ círculo, como na figura 2.7, e $X=M^{\prime} \backslash Y$. Seja $M$ um cone. Então, $g(Y)=$ vértice em $M$ e $g(X)=M \backslash$ vértice.

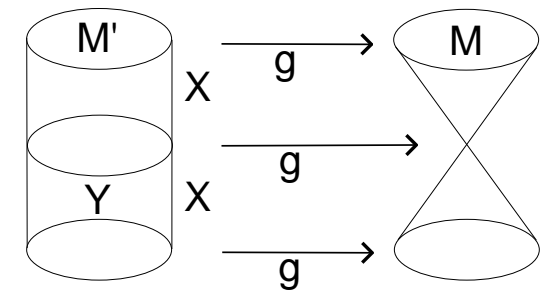

Figura 2.7: condição de Thom

Nesta situação, a condição $\left(a_{g}\right)$ de Thom não é satisfeita. De fato, considere a figura abaixo:

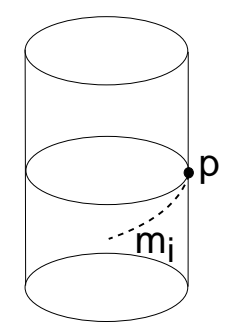

Figura 2.8:

Seja $\left\{m_{i}\right\}$ uma seqüência de pontos em $X$ tal que $m_{i} \rightarrow p$ quando $i \rightarrow \infty$. Então:

$\left.d g\right|_{X}\left(m_{i}\right): T_{m_{i}} X \cong \mathbb{R}^{2} \longrightarrow T_{g\left(m_{i}\right)} g(X) \cong \mathbb{R}^{2}$ é um isomorfismo. Logo, $\operatorname{ker}\left(\left.d g\right|_{X}\left(m_{i}\right)\right)=0$.

Agora, como $\left.d g\right|_{Y}(p) \equiv 0$, temos que $\operatorname{ker}\left(\left.d g\right|_{Y}(p)\right)=T_{p} Y$ que, por sua vez, tem dimensão 1. Assim, $\operatorname{ker}\left(\left.d g\right|_{Y}(p)\right) \not \subset \tau=\lim _{i \rightarrow \infty} \operatorname{ker}\left(\left.d g\right|_{X}\left(m_{i}\right)\right)=0$.

Portanto, este exemplo não satisfaz a condição $\left(a_{g}\right)$ de Thom. 
Agora, considere novamente o seguinte diagrama:

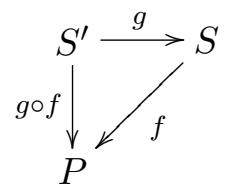

Definição 2.12. Dizemos que g é uma aplicação de Thom (sobre P) se as seguintes condições são satisfeitas:

(a) $\left.g\right|_{S^{\prime}}$ e $\left.f\right|_{S}$ são próprias;

(b) para cada estrato $X$ de $S,\left.f\right|_{X}$ é uma submersão;

(c) para cada estrato $X^{\prime}$ de $S^{\prime}, g\left(X^{\prime}\right) \subset X$ e $g: X^{\prime} \longrightarrow X$ é uma submersão (logo $\left.g\right|_{X^{\prime}}$ tem posto constante);

(d) qualquer par $\left(X^{\prime}, Y^{\prime}\right)$ de estratos de $S^{\prime}$ satisfaz a condição $\left(a_{g}\right)$ de Thom (a qual faz sentido devido ao ítem (c)).

Teorema 2.7. (Segundo Lema de Isotopia Thom, [15] e [21]): Se g é uma aplicação de Thom sobre $P$, então $g$ é localmente trivial sobre $P$. 


\section{Capítulo 3}

\section{Condição de regularidade de K. Bekka e Condição Whitney fraca}

Neste capítulo, introduziremos a (c)-regularidade definida por K. Bekka em [1], a qual implica trivialidade topológica local. Apresentaremos também uma condição de regularidade conhecida por Whitney fraca, definida por D. Trotman e K. Bekka em [3], e mostraremos que os espaços com uma estratificação Whitney fraca formam uma classe intermediária entre a estratificação $(b)$ de Whitney e a (c)-regularidade de Bekka.

\subsection{A (c)-regularidade de K. Bekka}

Sejam $M$ uma variedade regular, $A \subset M$ um subconjunto subanalítico com uma estratificação subanalítica $S$, e considere $Y$ um estrato. Seja $\operatorname{Star}(Y)$ o subconjunto de $S$ definido por:

$$
\operatorname{Star}(Y)=\{X \in S ; Y \subset \bar{X}\}
$$

Suponhamos que, para todo $p \in Y$, existe uma função controle $\rho_{p}$ definida numa vizinhança $U_{p}$ de $p$ em $M$, tal que $\rho_{p}$ define a (c)-regularidade localmente, que é como segue.

Definição 3.1. Dizemos que o par $(X, Y)$ satisfaz a condição $(c)$-regular, localmente em $p \in Y$, se as condições a seguir são satisfeitas:

(1) $\rho_{p}$ é uma função não negativa, subanalítica e de classe $C^{k}, k \geq 2$;

(2) $\rho_{p}^{-1}(0)=Y \cap U_{p}$;

(3) $\left.\rho_{p}\right|_{X}$ é uma submersão para todo $X \in \operatorname{Star}(Y)$;

(4) Para toda seqüência de pontos $\left\{m_{i}\right\}$ em $X$, tal que $m_{i} \rightarrow p \in Y$ e $\operatorname{ker}\left(\left.d \rho_{p}\right|_{X}\left(m_{i}\right)\right) \rightarrow \tau$, então $T_{p} Y \subset \tau$.

Proposição 3.1. $(A, S)$ é (a)-regular. 
Demonstração: De fato, (a)-regularidade é uma condição local, e visto que $(c)$ implica $(a)$ (item (4) da definição de (c)-regularidade), obtemos (a)-regularidade como uma conseqüência.

Observação 3.1. Suponhamos que exista uma função controle $\rho$ definida numa vizinhança aberta do estrato $Y$ em $M$, tal que:

- $\rho$ é uma função não negativa, subanalítica e de classe $C^{k}, k \geq 2$;

- $\rho^{-1}(0)=Y$.

Diremos, simplesmente, que o par $(X, Y)$ é (c)-regular (ou $(c)$-regular globalmente), se $\rho$ satisfizer os ítens (3) e (4) da definição 3.1.

Note que, a propriedade (3) da definição 3.1 diz que $\left.\rho_{p}\right|_{S t a r(Y)}$ é uma aplicação estratificada, isto é, $\left.\rho_{p}\right|_{\operatorname{Star}(Y)}$ é uma submersão. Além disso, a propriedade (4) diz que $\left.\rho_{p}\right|_{\text {Star(Y) }}$ é uma aplicação de Thom. Mais claramente, temos:

Proposição 3.2. A propriedade (4) da definição 3.1 é equivalente à

$$
\lim _{m \rightarrow p} \pi_{p}\left(\frac{\left.\nabla_{m} \rho_{p}\right|_{X}}{\left\|\left.\nabla_{m} \rho_{p}\right|_{X}\right\|}\right)=0
$$

para todo $X \in \operatorname{Star}(Y)$, em que $\pi_{p}$ é a projeção ortogonal sobre $T_{p} Y$ e $0 \in T_{p} Y$.

Demonstração: Como $(X, Y)$ é $(a)$-regular, temos que $\lim _{m \rightarrow p} T_{m} X=T \supset Y$ (podemos supor que $Y$ é linear).

Visto que $\left.\rho_{p}\right|_{X}$ é uma submersão, temos que $\operatorname{ker}\left(\left.d \rho_{p}\right|_{X}(m)\right)$ é um hiperplano em $T_{m} X$ e, como

$$
\frac{\left.\nabla_{m} \rho_{p}\right|_{X}}{\left\|\left.\nabla_{m} \rho_{p}\right|_{X}\right\|}
$$

é ortogonal ao subespaço $\operatorname{ker}\left(\left.d \rho_{p}\right|_{X}(p)\right)$, temos que

$$
\lim _{m \rightarrow p} \frac{\left.\nabla_{m} \rho_{p}\right|_{X}}{\left\|\left.\nabla_{m} \rho_{p}\right|_{X}\right\|}
$$

é ortogonal a $\tau=\lim _{m \rightarrow p} \operatorname{ker}\left(\left.d \rho_{p}\right|_{X}(m)\right)$. Então,

$$
\lim _{m \rightarrow p} \pi_{p}\left(\frac{\left.\nabla_{m} \rho_{p}\right|_{X}}{\left\|\left.\nabla_{m} \rho_{p}\right|_{X}\right\|}\right)=0 \Longrightarrow Y \subset \tau
$$

A recíproca é provada de maneira análoga, voltando.

Em [2], Bekka provou que dados dois pontos $p_{1}, p_{2} \in Y$ e funções controle $\rho_{p_{1}}: U_{1} \longrightarrow \mathbb{R}_{+}$e $\rho_{p_{2}}: U_{2} \longrightarrow \mathbb{R}_{+}$tal que $U_{1} \cap U_{2} \neq \emptyset$, satisfazendo a $(c)$-regularidade em $p_{1}$ e $p_{2}$, respectivamente, é possível construir uma função controle $\rho: U_{1} \cup U_{2} \longrightarrow \mathbb{R}_{+}$que também satisfaz a $(c)$-regularidade em todo $p \in U_{1} \cup U_{2}$, ou seja, (c)-regularidade local em todo ponto implica (c)-regularidade global. 
Teorema 3.1. Dado um par de estratos $(X, Y)$ com $Y \subset \bar{X}$ satisfazendo a definição 3.1 para todo $p \in Y$, temos que $(X, Y)$ é $(c)$-regular, ou seja, existe uma função controle $\rho$ satisfazendo a observação 3.1 .

Observação 3.2. (1) A condição (c) é invariante por difeomorfismo;

(2) (c)-regularidade implica trivialidade topológica local ao longo dos estratos, por integração de campos de vetores;

(3) Um par de estratos $(X, Y)$ ser (c)-regular significa que $\operatorname{dim} X \geq \operatorname{dim} Y+1$. Logo, uma estratificação $(c)$-regular é uma filtração pela dimensão;

(4) Se um espaço admite uma estratificação (c)-regular, então ele é triangularizável.

Para provar os resultados acima, o autor em [1] utiliza várias ferramentas abstratas, como sistemas de tubos, bons tubos, sistemas controlados de tubos e campos de vetores controlados. Não trataremos em profundidade esses resultados, pois as ferramentas utilizadas fogem da abordagem que pretendemos dar aqui.

\subsection{Estratificação $(c)$-regular de conjuntos subanalíticos}

Nesta seção, apresentaremos o resultado que garante a existência de uma estratificação (c)-regular para conjuntos subanalíticos. A prova desse resultado depende fortemente da proposição a seguir.

Proposição 3.3. Seja $M$ uma variedade regular analítica. Sejam $g: M \longrightarrow \mathbb{R}$ uma função subanalítica e de classe $C^{k}$ e $E_{\lambda}$ uma família localmente finita de conjuntos subanalíticos de $M$. Então existe uma estratificação subanalítica de $M$, compatível com $E_{\lambda}$, que satisfaz a condição $\left(a_{g}\right)$ de Thom.

Observação 3.3. (1) Podemos supor que $g$ é a restrição à $M$ de uma função analítica em $M \times \mathbb{R}$

(2) Uma estratificação $\left(a_{g}\right)$-regular de $\operatorname{Graf}(g)$ dá uma estratificação $\left(a_{g}\right)$-regular em $M$, já que esta condição é invariante por difeomorfismos;

(3) Desde que os valores singulares de $g$ formem um subconjunto subanalítico próprio de $\mathbb{R}$, isso implica que eles são pontos isolados em $\mathbb{R}$. Portanto, podemos supor que o conjunto dos valores singulares é reduzido a um ponto, digamos 0 . Já temos uma estratificação de $M$ por $\left(g^{-1}(0), M \backslash g^{-1}(0)\right)$. A condição $\left(a_{g}\right)$ sobre $g^{-1}(0)$ é somente a condição $(a)$ de Whitney, neste caso, o resultado é bem conhecido (ver [25]);

(4) A prova da Proposição 3.3 para funções analíticas complexas está em [10] Hamm e Lê (usa-se a desigualdade de Lojasiewicz e a existência de uma estratificação (a)-regular). 
Em [2], Bekka provou a Proposição 3.3 utilizando algumas ferramentas, tais como, a condição de regularidade " $(*)$-stratifing", o teorema de Puiseux com parâmetros, as quais não fazem parte dos objetivos do presente trabalho. Logo, não provaremos tal proposição.

Teorema 3.2. Todo conjunto subanalítico admite uma estratificação (c)-regular, onde as funções controle são as funções distância aos estratos.

O exemplo abaixo foi apresentado por Bekka para mostrar que a condição (b) de Whitney é mais forte que a $(c)$-regularidade de K. Bekka.

Exemplo 3.1. Seja $f(x, y, z, t)=z^{5}+t y^{6} z+y^{7} x+x^{15}$. Em [6] Briançon e Speder provaram que a variedade

$$
V=\left\{(x, y, z, t) \in \mathbb{K}^{4} ; f(x, y, z, t)=0\right\} \quad(\text { onde } \mathbb{K}=\mathbb{C} \text { ou } \mathbb{R}),
$$

estratificada por $Y=$ eixo $t$ e $X=V \backslash Y$, não satisfaz a condição $(b)$ de Whitney na origem, mas é topologicamente trivial ao longo do estrato $Y$.

Em [2], Bekka mostrou que o par de estratos $(X, Y)$ acima é $(c)$-regular com respeito a função controle $\rho$ dada por: $\rho(x, y, z, t)=|x|^{2}+|y|^{2}+|z|^{2}$. Para isso, ele utilizou uma $\mathbb{K}^{*}$-ação definida por $T_{\lambda}(x, y, z, t)=\left(\lambda x, \lambda^{2} y, \lambda^{3} z, t\right)$.

\subsection{A condição $(\delta)$}

Nesta seção, introduziremos uma condição métrica $(\delta)$, satisfeita por toda estratificação de Whitney. Estratificações satisfazendo a condição de fronteira, a condição $(a)$ de Whitney e a condição $(\delta)$ são chamadas de estratificações Whitney fraca. Estudaremos a seguir espaços satisfazendo as condições $(a)$ e $(\delta)$.

Definição 3.2. Seja $W$ um espaço vetorial euclideano. Dados $v_{1}, v_{2} \in W^{*}=W \backslash\{0\}$ definimos o seno do ângulo entre os dois vetores por:

$$
\sin \theta\left(v_{1}, v_{2}\right)=\frac{\left\|v_{1} \wedge v_{2}\right\|}{\left\|v_{1}\right\| \cdot\left\|v_{2}\right\|}
$$

onde $\wedge$ é o produto vetorial e $\|$.$\| é a norma induzida pela estrutura euclideana.$

Definição 3.3. Se $S$ e $T$ são subespaços de $W$, definimos o seno do ângulo entre eles por:

$$
\sin \theta(S, T)=\sup \left\{\sin \theta(s, T) ; s \in S^{*}\right\}
$$

onde $\sin \theta(s, T)=\inf \left\{\sin \theta(s, t) ; t \in T^{*}\right\}$.

Observação 3.4. Note que, $\sin \theta(s, T)=\frac{\left\|\pi_{T}(s)\right\|}{\|s\|}$, onde $\pi_{T}: W \longrightarrow T^{\perp}$ é a projeção ortogonal sobre o complemento ortogonal de $T$.

Temos as seguintes propriedades:

(i) $\sin \theta\left(v_{1}, v_{3}\right) \leq \sin \theta\left(v_{1}, v_{2}\right)+\sin \theta\left(v_{2}, v_{3}\right)$, para todo $v_{1}, v_{2}, v_{3} \in W^{*}$; 
(ii) $\sin \theta\left(S_{1}+S_{2}, T\right) \leq \sin \theta\left(S_{1}, T\right)+\sin \theta\left(S_{2}, T\right)$, onde $S_{1}, S_{2}$ e $T$ são subespaços de $W$, com $S_{1}$ ortogonal a $S_{2}$.

Sejam $X$ e $Y$ subvariedades regulares de uma variedade regular $M$ e $p \in Y$.

Definição 3.4. Dizemos que $X$ é $(\delta)$-regular sobre $Y$ no ponto $p$, se existem um difeomorfismo local $h: \mathbb{R}^{n} \longrightarrow M$ para uma vizinhança $U$ de $p$ em $M$, e um número real $\delta_{p}$, com $0 \leq \delta_{p}<1$, tais que, para cada seqüência $\left\{\left(m_{i}, p_{i}\right)\right\} \in h^{-1}(X) \times h^{-1}(Y)$ convergindo para $\left(h^{-1}(p), h^{-1}(p)\right)$, com $\widehat{m_{i} p_{i}}$ convergindo para l em $\mathbb{P}^{n-1}$ e $T_{m_{i}} h^{-1}(X) \longrightarrow \tau$, tem-se: $\sin \theta(l, \tau) \leq \delta_{p}$.

Observação 3.5. Visto que a definição acima depende de $h$, dizemos também que $(X, Y)$ satisfaz a condição $(\delta)$ para o difeomorfismo local $h$ em $p$.

Definição 3.5. Dizemos que o par $(X, Y)$ satisfaz a condição $(\delta)$, se ele satisfaz $(\delta)$ em todo ponto de $Y$.

Observação 3.6. A condição $(b)$ de Whitney implica a condição $(\delta)$, basta tomarmos $\delta_{p}=0$, pois neste caso $l \subset \tau$. No final da seção 3.5, apresentaremos exemplos para mostrar que as condições $(a)$ e $(\delta)$ são independentes.

\subsection{Estratificações Whitney fraca}

Definição 3.6. Sejam $M$ uma variedade regular e $V \subset M$. Uma estratificação Whitney fraca de $V$ é uma estratificação de $V$ satisfazendo as seguintes condições:

(1) Condição de fronteira;

(2) Todo par de estratos adjacentes satisfaz a condição (a) de Whitney;

(3) Todo par de estratos adjacentes satisfaz a condição $(\delta)$, isto é, para todo estrato $Y$, existem uma vizinhança $U$ de $Y$ e um difeomorfismo $h: W \subset \mathbb{R}^{n} \longrightarrow U$ tal que, para todo $X \in \operatorname{Star}(Y)$ e $p \in Y$, o par $(X, Y)$ satisfaz a condição $(\delta)$ para $h$ em $p$.

Exemplo 3.2. Toda estratificação de Whitney é Whitney fraca. De fato, pelas Proposições 2.1 e 2.2 obtemos a condição de regularidade $(a)$ e a condição de fronteira, respectivamente, e pela observação 3.6 temos a condição $(\delta)$.

Na definição da condição Whitney fraca, podemos enfraquecer a condição $(\delta)$ da seguinte maneira: Seja $\pi: T \longrightarrow Y$ uma $C^{1}$-retração local associada a uma $C^{1}$-vizinhança tubular $T$ de $Y$ próximo de $p$.

Definição 3.7. Dizemos que $X$ é $\left(\delta^{\pi}\right)$-regular sobre $Y$ no ponto $p \in Y$, se existem um difeomorfismo local $h: \mathbb{R}^{n} \longrightarrow M$ de uma vizinhança $U$ de $p$ em $M$ e um número real $\delta_{p}, 0 \leq \delta_{p}<1$, tais que para cada seqüência $\left\{\left(m_{i}, \pi\left(m_{i}\right)\right)\right\} \in h^{-1}(X) \times h^{-1}(Y)$ convergindo para $\left(h^{-1}(p), h^{-1}(p)\right)$, com $\left.\widehat{m_{i} \pi\left(m_{i}\right.}\right) \rightarrow l$ em $\mathbb{P}^{n-1}$ e $T_{m_{i}} h^{-1}(X) \rightarrow \tau$, tem-se: $\sin \theta(l, \tau) \leq \delta_{p}$. 
Proposição 3.4. $\left(\delta^{\pi}\right)+(a) \Leftrightarrow(\delta)+(a)$

Demonstração: Obviamente $(\delta)+(a) \Rightarrow\left(\delta^{\pi}\right)+(a)$, já que $(\delta) \Rightarrow\left(\delta^{\pi}\right)$.

Mostremos que $\left(\delta^{\pi}\right)+(a) \Rightarrow(\delta)$, provando que $\left(\delta^{\pi}\right)+(a)=(\delta)+(a)$. Na definição de $(\delta)$, decomponha o limite do vetor unitário $l$ como um vetor $l_{1}$ tangente a $Y$ em $p$ e um vetor $l_{2}$ tangente a $\pi^{-1}(p)$ em $p$.

Como $\sin \theta(l, \tau)=\sin \theta\left(l_{1}+l_{2}, \tau\right) \leq \sin \theta\left(l_{1}, \tau\right)+\sin \theta\left(l_{2}, \tau\right)$, e pela condição $(a)$, temos que $\sin \theta\left(l_{1}, \tau\right)=0$, obtemos a desigualdade $\sin \theta(l, \tau) \leq \sin \theta\left(l_{2}, \tau\right)$. Mas, da condição $\left(\delta^{\pi}\right)$, segue-se que $\sin \theta\left(l_{2}, \tau\right) \leq \delta_{p}$. Logo, $\sin \theta(l, \tau) \leq \delta_{p}$, obtendo a condição $(\delta)$.

\subsection{Exemplos algébricos reais}

Exemplo 3.3. Aqui, temos um exemplo onde as condições $(a)$ e $(\delta)$ valem, mas $(b)$ não é satisfeita. Tal exemplo mostra que a condição $(b)$ de Whitney é mais forte que a condição Whitney fraca.

Seja $f_{1}(x, y, z)=-y^{6}+z^{6} x^{2}+x^{6}$; então, a variedade $V_{1}$ definida por $f_{1}$ é $V_{1}=\left\{(x, y, z) \in \mathbb{R}^{3} ; f_{1}(x, y, z)=0\right\}$. Sejam $Y=$ eixo $z$ e $X=V_{1} \backslash Y$.

Afirmamos que $(X, Y)$ satisfaz as condições $(a)$ e $(\delta)$ em $p=(0,0,0) \in Y$, mas não satisfaz a condição $(b)$ de Whitney. De fato, seja $m=(x, y, z) \in X,(x, y) \neq(0,0)$; então, o espaço normal é gerado por:

$$
\nabla f_{1}(x, y, z)=\left(2 x z^{6}+6 x^{5},-6 y^{5}, 6 x^{2} z^{5}\right)
$$

O vetor nornal (direção) é:

$$
v(m)=\frac{\nabla f_{1}(x, y, z)}{\left\|\nabla f_{1}(x, y, z)\right\|}=\left(\frac{2 x z^{6}+6 x^{5}}{\sqrt{B_{1}}}, \frac{-6 y^{5}}{\sqrt{B_{1}}}, \frac{\left.6 x^{2} z^{5}\right)}{\sqrt{B_{1}}}\right)
$$

onde $B_{1}=\left(2 x z^{6}+6 x^{5}\right)^{2}+36 y^{10}+36 x^{4} z^{10}$.

Agora, se $\tau \in T_{p} Y$, podemos assumir que $\tau=(0,0,1)$, visto que $T_{p} Y$ é 1-dimensional. Logo,

$$
\left|\pi_{N_{m} X}(\tau)\right|=|v(m) \cdot \tau|=\left|\frac{6 x^{2} z^{5}}{\sqrt{\left(2 x z^{6}+6 x^{5}\right)^{2}+36 y^{10}+36 x^{4} z^{10}}}\right| \leq\left|\frac{6 x^{2} z^{5}}{2 x z^{6}+6 x^{5}}\right|=\left|\frac{3 x z^{5}}{z^{6}+3 x^{4}}\right| .
$$

Seja $m_{i}=\left(x_{i}, y_{i}, z_{i}\right)$ uma seqüência de pontos em $X$ convergindo para $(0,0,0)$.

Se $\frac{\left|z_{i}\right|}{\left|x_{i}\right|} \leq K<\infty$, então:

$$
\left|v\left(m_{i}\right) \cdot \tau\right| \leq\left|\frac{3 x_{i} z_{i}^{5}}{z_{i}^{6}+3 x_{i}^{4}}\right| \leq\left|\frac{3 x_{i} z_{i}^{5}}{3 x_{i}^{4}}\right|=\frac{\left|z_{i}\right|^{4}}{\left|x_{i}\right|^{4}}\left|x_{i} z_{i}\right| \longrightarrow 0 \text { quando } x_{i} \rightarrow 0
$$

Se $\frac{\left|x_{i}\right|}{\left|z_{i}\right|} \longrightarrow 0$, temos:

$$
\left|v\left(m_{i}\right) \cdot \tau\right| \leq\left|\frac{3 x_{i} z_{i}^{5}}{z_{i}^{6}+3 x_{i}^{4}}\right| \leq\left|\frac{3 x_{i} z_{i}^{5}}{z_{i}^{6}}\right|=3 \frac{\left|x_{i}\right|}{\left|z_{i}\right|} \longrightarrow 0
$$


Portanto, em cada caso vimos que a condição $(a)$ vale. Note que a condição $(a)$ é satisfeita em todo ponto de $Y$, pois se $z \neq 0$ temos que o lado direito da desigualdade abaixo tende a zero quando $(x, y)$ tende à $(0,0)$ :

$$
\left|\pi_{N_{m} X}(\tau)\right|=|v(m) \cdot \tau| \leq\left|\frac{6 x^{2} z^{5}}{2 x z^{6}+6 x^{5}}\right|=\left|\frac{3 x z^{5}}{z^{6}+3 x^{4}}\right| .
$$

Vamos verificar a seguir que $(X, Y)$ satisfaz a condição $(\delta)$ em $p$.

Como a condição $(a)$ vale em todo ponto de $Y$, pela Proposição 3.4 é suficiente verificarmos a condição $\left(\delta^{\pi}\right)$, onde $\pi: \mathbb{R}^{3} \longrightarrow Y$ é dada por $\pi(x, y, z)=(0,0, z)$. Temos que:

$$
\widehat{\pi\left(m_{i}\right) m_{i}}=\left(x_{i}, y_{i}, 0\right) \Longrightarrow \frac{\pi \widehat{\left(m_{i}\right) m_{i}}}{\left\|\widetilde{\pi\left(m_{i}\right) m_{i}}\right\|}=\frac{\left(x_{i}, y_{i}, 0\right)}{\sqrt{x_{i}^{2}+y_{i}^{2}}} .
$$

Logo,

$$
\frac{\left|v\left(m_{i}\right) \cdot \widehat{\pi\left(m_{i}\right)} m_{i}\right|}{\left\|\pi \widehat{\left(m_{i}\right) m_{i}}\right\|}=\left|\frac{2 x_{i}^{2} z_{i}^{6}+6 x_{i}^{6}-6 y_{i}^{6}}{\sqrt{\left(2 x_{i} z_{i}^{6}+6 x_{i}^{5}\right)^{2}+36 y_{i}^{10}+36 x_{i}^{4} z_{i}^{10}} \sqrt{x_{i}^{2}+y_{i}^{2}}}\right| .
$$

Elevando ambos os membros ao quadrado, temos:

$$
\begin{aligned}
\left(\frac{\mid v\left(m_{i}\right) \cdot{\widehat{\pi\left(m_{i}\right) m_{i}}}_{i}}{\left\|\pi \widehat{\left(m_{i}\right) m_{i}}\right\|}\right)^{2} & =\left|\frac{\left(2 x_{i}^{2} z_{i}^{6}+6 x_{i}^{6}-6 x_{i}^{2} z_{i}^{6}-6 x_{i}^{6}\right)^{2}}{\left[\left(2 x_{i} z_{i}^{6}+6 x_{i}^{5}\right)^{2}+36 y_{i}^{10}+36 x_{i}^{4} z_{i}^{10}\right]\left[x_{i}^{2}+y_{i}^{2}\right]}\right| \\
& \leq\left|\frac{\left(4 x_{i}^{2} z_{i}^{6}\right)^{2}}{36 y_{i}^{10}\left(x_{i}^{2}+y_{i}^{2}\right)}\right| \\
& \leq\left|\frac{\left(4 x_{i}^{2} z_{i}^{6}+4 x_{i}^{6}\right)^{2}}{36 y_{i}^{10}\left(x_{i}^{2}+y_{i}^{2}\right)}\right| \\
& =\left|\frac{\left(4 y_{i}^{6}\right)^{2}}{36 y_{i}^{10}\left(x_{i}^{2}+y_{i}^{2}\right)}\right| \\
& =\left|\frac{4 y_{i}^{2}}{9\left(x_{i}^{2}+y_{i}^{2}\right)}\right| \leq \frac{4}{9}
\end{aligned}
$$

Assim, a condição $(\delta)$ vale para $\delta_{p}=\frac{2}{3}$.

Finalmente, mostremos que $(X, Y)$ não é $(b)$-regular.

Afirmamos que $(b)$ falha ao longo da curva $\gamma(t)=\left(t^{\frac{3}{2}}, \sqrt[6]{2} \cdot t^{\frac{3}{2}}, t\right) \in V_{1}$. De fato, temos que:

$$
\pi\left(\widehat{\gamma(t)) \gamma}(t)=\left(t^{\frac{3}{2}}, \sqrt[6]{2} \cdot t^{\frac{3}{2}}, 0\right) \Longrightarrow \frac{\pi(\widehat{\gamma(t)) \gamma}(t)}{\| \pi(\widehat{\gamma(t)) \gamma}(t) \|}=\frac{\left(t^{\frac{3}{2}}, \sqrt[6]{2} \cdot t^{\frac{3}{2}}, 0\right)}{\sqrt{t^{3}+\sqrt[3]{2} \cdot t^{3}}}\right.
$$

Logo,

$$
\frac{\mid v(\gamma(t)) \cdot \pi(\widehat{\gamma(t)) \gamma}(t) \mid}{\| \pi(\widehat{\gamma(t)) \gamma}(t) \|}=\left|\left\langle\frac{\left(2 t^{\frac{15}{2}}+6 t^{\frac{15}{2}},-6 \sqrt[6]{2^{5}} \cdot t^{\frac{15}{2}}, 6 t^{8}\right)}{\sqrt{64 t^{15}+36 \sqrt[3]{2^{5}} \cdot t^{15}+36 t^{16}}}, \frac{\left(t^{\frac{3}{2}}, \sqrt[6]{2} \cdot t^{\frac{3}{2}}, 0\right)}{\sqrt{t^{3}+\sqrt[3]{2} \cdot t^{3}}}\right\rangle\right|
$$




$$
=\left|\frac{8 t^{9}-12 t^{9}}{\left[64 t^{15}+36 \sqrt[3]{2^{5}} \cdot t^{15}+36 t^{16}\right]^{\frac{1}{2}}\left[(1+\sqrt[3]{2}) t^{3}\right]^{\frac{1}{2}}}\right| .
$$

Elevando ao quadrado, temos:

$$
\begin{aligned}
\left(\frac{\mid v(\gamma(t)) \cdot \pi(\widehat{\gamma(t)) \cdot \gamma}(t) \mid}{\| \pi(\widehat{\gamma(t)) \cdot \gamma}(t) \|}\right)^{2} & =\left|\frac{16 t^{18}}{4 t^{15}\left(16+9.2^{\frac{5}{3}}+9 t\right)\left[\left(1+2^{\frac{1}{3}}\right) t^{3}\right]}\right| \\
& =\left|\frac{4}{\left(16+9 \cdot 2^{\frac{5}{3}}+9 t\right)\left(1+2^{\frac{1}{3}}\right)}\right| \nrightarrow 0 \text { quando } t \rightarrow 0
\end{aligned}
$$

Portanto, $(X, Y)$ não é (b)-regular.

A seguir apresentaremos alguns exemplos para mostrar que as condições de regularidade $(a)$ e $(\delta)$ são independentes.

Exemplo 3.4. Mostraremos através deste exemplo que a condição de regularidade $(\delta)$ não implica a condição de regularidade $(a)$.

Seja $f_{2}(x, y, z)=-y^{20}+z^{4} x^{6}+x^{10}$; então, a variedade algébrica $V_{2}$ definida por $f_{2}$ é $V_{2}=\left\{(x, y, z) \in \mathbb{R}^{3} ; f_{2}(x, y, z)=0\right\}$. Sejam $Y=$ eixo $z$ e $X=V_{2} \backslash Y$.

Afirmação 1: a condição $(a)$ falha em $p=(0,0,0) \in Y$.

De fato, sabemos que para todo $m=(x, y, z) \in X, \operatorname{com}(x, y) \neq(0,0)$, a direção do vetor normal é:

$$
v(m)=\frac{\nabla f_{2}(x, y, z)}{\left\|\nabla f_{2}(x, y, z)\right\|}=\left(\frac{6 x^{5} z^{4}+10 x^{9}}{\sqrt{B_{2}}}, \frac{-20 y^{19}}{\sqrt{B_{2}}}, \frac{4 x^{6} z^{3}}{\sqrt{B_{2}}}\right),
$$

onde $B_{2}=\left(6 x^{5} z^{4}+10 x^{9}\right)^{2}+\left(20 y^{19}\right)^{2}+16 x^{12} z^{6}$.

Seja $\tau \in T_{p} Y$, então podemos assumir que $\tau=(0,0,1)$, já que $T_{p} Y$ é 1 -dimensional. Temos que:

$$
\left|\pi_{N_{m} X}(\tau)\right|=|v(m) \cdot \tau|=\left|\frac{4 x^{6} z^{3}}{\sqrt{\left(6 x^{5} z^{4}+10 x^{9}\right)^{2}+\left(20 y^{19}\right)^{2}+16 x^{12} z^{6}}}\right| .
$$

A curva utilizada pelos autores, em [3], para provar que a condição $(a)$ falha é $\gamma(t)=\left(t, t^{\frac{1}{2}}, t\right)$ mas, esta curva não pertence a variedade $V_{2}$; portanto, precisamos ajustar tal curva. Considere a curva $\gamma(t)=\left(t, \sqrt[20]{2} \cdot t^{\frac{1}{2}}, t\right) \in V_{2}$, então:

$$
\begin{aligned}
|v(\gamma(t)) \cdot \tau| & =\left|\frac{4 t^{6} t^{3}}{\sqrt{\left(6 t^{5} t^{4}+10 t^{9}\right)^{2}+\left(20 \sqrt[20]{2^{19}} \cdot t^{\frac{19}{2}}\right)^{2}+16 t^{12} t^{6}}}\right| \\
& =\left|\frac{4 t^{9}}{\left.\left(16 t^{9}\right)^{2}+20^{2} \cdot 2^{\frac{19}{10}} t^{19}+16 t^{18}\right]^{\frac{1}{2}}}\right| .
\end{aligned}
$$

Elevando ambos os membros ao quadrado, temos:

$$
|v(\gamma(t)) \cdot \tau|^{2}=\left|\frac{16 t^{18}}{256 t^{18}+20^{2} \cdot 2^{\frac{19}{10}} t^{19}+16 t^{18}}\right|=\left|\frac{16}{272+20^{2} \cdot 2^{\frac{19}{10}}}\right| .
$$


Logo, $\lim _{\gamma(t) \rightarrow p}|v(\gamma(t)) \cdot \tau|=\lim _{t \rightarrow 0}\left|\frac{16}{272+20^{2} .2^{\frac{19}{10}} t}\right|^{\frac{1}{2}}=\frac{1}{\sqrt{17}} \neq 0$. Portanto, a condição $(a)$ falha.

Afirmação 2: a condição $(\delta)$ é satisfeita em todo ponto de $Y$.

Decorre direto de manipulações das contas e será deixado a cargo do leitor.

Exemplo 3.5. (Exemplo 2.4, Capítulo 2) Este exemplo mostra que a condição de regularidade (a) não implica a condição de regularidade $(\delta)$.

Lembre-se que $V_{4}=\left\{(x, y, z) \in \mathbb{R}^{3} ; f_{4}(x, y, z)=0\right\}$, onde $f_{4}(x, y, z)=y^{2}-z^{2} x^{2}-x^{3}$. Sejam $Y=$ eixo $z, X=V_{4} \backslash Y$ e $p=(0,0,0) \in Y$.

Do capítulo 2, temos que o par de estratos $(X, Y)$ é $(a)$-regular. Além disso, sabemos que para todo $m=(x, y, z) \in X, \operatorname{com}(x, y) \neq(0,0)$, o espaço normal é gerado por:

$$
\nabla f_{4}(x, y, z)=\left(-2 x z^{2}-3 x^{2}, 2 y,-2 x^{2} z\right)
$$

E, o vetor normal (direção) é:

$$
v(m)=\frac{\nabla f_{4}(x, y, z)}{\left|\nabla f_{4}(x, y, z)\right|}=\left(\frac{-2 x z^{2}-3 x^{2}}{\sqrt{A_{4}}}, \frac{2 y}{\sqrt{A_{4}}}, \frac{-2 x^{2} z}{\sqrt{A_{4}}}\right)
$$

onde $A_{4}=\left(-2 x z^{2}-3 x^{2}\right)^{2}+4 y^{2}+4 x^{4} z^{2}$.

A seguir mostraremos que o par de estratos $(X, Y)$ não satisfaz a condição $(\delta)$ em $p$.

Observe que, como $(\delta) \Rightarrow\left(\delta^{\pi}\right)$, basta mostrarmos que $(X, Y)$ não satisfaz a condição $\left(\delta^{\pi}\right)$, onde $\pi: \mathbb{R}^{3} \longrightarrow Y$ é definida por $\pi(x, y, z)=(0,0, z)$.

Afirmamos que $\left(\delta^{\pi}\right)$ falha ao longo da curva $\alpha(t)=\left(-t^{2}, 0, t\right) \in V_{4}$. De fato, temos que:

$$
\pi\left(\widehat{\alpha(t)) \alpha}(t)=\left(-t^{2}, 0,0\right) \Longrightarrow \frac{\pi(\widehat{\alpha(t)) \alpha}(t)}{\| \pi(\widehat{\alpha(t)) \alpha}(t) \|}=\frac{\left(-t^{2}, 0,0\right)}{\sqrt{\left(-t^{2}\right)^{2}+0}}=(-1,0,0)\right.
$$

Logo,

$$
\begin{aligned}
\frac{\mid v(\alpha(t)) \cdot \pi(\widehat{\alpha(t))} \alpha(t) \mid}{\| \pi(\widehat{\alpha(t)) \alpha}(t) \|} & =\left|\frac{-1\left[-2\left(-s^{2}\right) s^{2}-3\left(s^{2}\right)^{2}\right]}{\sqrt{\left[2\left(-s^{2}\right) s^{2}+3\left(-s^{2}\right)^{2}\right]^{2}+4 \cdot 0+4\left(-s^{2}\right)^{4} s^{2}}}\right| \\
& =\left|\frac{-\left(2 s^{4}-3 s^{4}\right)}{\sqrt{\left(-2 s^{4}+3 s^{4}\right)^{2}+4 s^{10}}}\right| \\
& =\left|\frac{-\left(-s^{4}\right)}{\sqrt{s^{8}+4 s^{10}}}\right| \\
& =\left|\frac{s^{4}}{s^{4} \sqrt{1+4 s^{2}}}\right|=\left|\frac{1}{\sqrt{1+4 s^{2}}}\right|=1, \text { quando } s \rightarrow 0 .
\end{aligned}
$$

Deste modo, segue-se que o par de estratos $(X, Y)$ não satisfaz a condição $\left(\delta^{\pi}\right) \operatorname{em} p=(0,0,0)$, pois $0 \leq \delta_{p}<1$ e, consequentemente, $(X, Y)$ não é $(\delta)$ - regular. 


\subsection{Propriedades dos espaços com uma estratificação Whitney fraca}

Nesta seção, mostraremos que um espaço que admite uma estratificação Whitney fraca é filtrado pela dimensão, isto é, o bordo topológico de um estrato $X$ tem dimensão estritamente menor que $X$. Isto nem sempre é verdade para estratificações $(w)$-regular (capítulo 4). Além disso, provaremos que os espaços Whitney fraco estratificados são $(c)$-regulares e portanto, localmente, ao longo dos estratos, são topologicamente triviais.

Proposição 3.5. Considere $(X, Y)$ uma par de estratos satisfazendo as condições $(a)$ e $(\delta)$ em $p \in \bar{X} \cap Y$. Então, $\operatorname{dim} Y<\operatorname{dim} X$.

Demonstração: Sendo o problema de caráter local, podemos assumir que $X=\mathbb{R}^{n}$.

Como $p \in \bar{X} \cap Y$, existe uma seqüência de pontos $\left\{m_{i}\right\}$ em $X$ convergindo para $p$. Pela compacidade da variedade Grasmanniana, podemos supor, passando para uma subseqüência, se necessário, que $T_{m_{i}} X$ converge para um $r$ plano $\tau \subset \mathbb{R}^{n}$ (onde $r=\operatorname{dim} X$ ). Logo, pela condição (a) de Whitney, temos que $T_{p} Y \subset \tau$, assim $\operatorname{dim} Y \leq \operatorname{dim} X$. Mas, para $i$ suficientemente grande, existe um ponto $p_{i} \in Y$ que minimiza a distância para $m_{i}$. Novamente utilizando uma subseqüência se necessário, podemos assumir que as secantes $\widehat{m_{i} p_{i}}$ convergem para uma reta $l \subset \mathbb{R}^{n}$. Como $p_{i}$ minimiza a distância para $m_{i}$, a secante $\widehat{m_{i} p_{i}}$ é ortogonal a $T_{p_{i}} Y$. Logo,

$$
l=\lim _{m_{i}, p_{i} \rightarrow p} \widehat{m_{i} p_{i}} \perp \lim _{p_{i} \rightarrow p} T_{p_{i}} Y=T_{p} Y .
$$

Como a condição $(\delta)$ assegura a existência de um número real $\delta_{p}, 0 \leq \delta_{p}<1$, tal que $\sin \theta(l, \tau) \leq \delta_{p}$; logo, a projeção $\pi_{\tau}(l)$ é não nula. Note que, como $l$ é ortogonal a $Y$, temos que $\pi_{\tau}(l) \not \subset Y$.

Portanto, deduzimos que: $\operatorname{dim} X=\operatorname{dim} \tau \geq \operatorname{dim}\left(l \oplus T_{p} Y\right)>\operatorname{dim} Y$.

Definição 3.8. Seja $(U, h)$ uma $C^{1}$-carta em $p$ para uma subvariedade regular $Y \subset M$, onde $\operatorname{dim} Y=d$, e seja

$$
h:(U, U \cap Y, p) \longrightarrow\left(\mathbb{R}^{n}, \mathbb{R}^{d} \times O^{n-d}, O\right),
$$

onde $O$ denota a origem do estrato.

Então, $h$ define uma vizinhança tubular $T_{h}$ de $U \cap Y$ em $U$, induzida pela vizinhança canônica de $\mathbb{R}^{d} \times O^{n-d}$ em $\mathbb{R}^{n}$ :

- com retração $\pi_{h}: h^{-1} \circ \pi_{d} \circ h$, onde $\pi_{d}: \mathbb{R}^{n} \longrightarrow \mathbb{R}^{d}$ é a projeção canônica;

- e a função distância $\rho_{h}=\rho \circ h: U \longrightarrow \mathbb{R}_{+}$, onde $\rho: \mathbb{R}^{n} \longrightarrow \mathbb{R}_{+}$é a função distância definida por: $\rho\left(x_{1}, \ldots, x_{n}\right)=\sum_{i=d+1}^{n} x_{i}^{2}$. 
Sabemos que, se o par $(X, Y)$ de subvariedades regulares de $M$ satisfizer a condição $(b)$ de Whitney, então para toda vizinhança tubular, suficientemente pequena, $T_{Y}$ de $Y$ em $M$, temos que $\left.\left(\pi_{Y}, \rho_{Y}\right)\right|_{X \cap T_{Y}}: X \cap T_{Y} \longrightarrow Y \times \mathbb{R}$ é uma submersão (ver [15], Lema 7.3).

A seguir, mostraremos que se um par de estratos $(X, Y)$ é Whitney fraco; então, existe alguma vizinhança tubular $T_{Y}$ tal que $\left.\left(\pi_{Y}, \rho_{Y}\right)\right|_{X \cap T_{Y}}: X \cap T_{Y} \longrightarrow Y \times \mathbb{R}$ é uma submersão. Essa propriedade é sempre satisfeita para estratificações $(c)$-regulares.

Proposição 3.6. Sejam $X, Y$ duas subvariedades regulares de $M$, com $Y \subset \bar{X}$, tal que o par $(X, Y)$ satisfaz as condições $(a)$ e $(\delta)$ em $p \in Y$. Então, existem uma $C^{1}$-carta $(U, h)$ de $p$ para $Y$ em $M$, e uma vizinhança $U^{\prime}$ de $p, U^{\prime} \subset U$, tal que $\left.\left(\pi_{h}, \rho_{h}\right)\right|_{U^{\prime} \cap X}$ é uma submersão.

Demonstração: Pela definição de $(\delta)$, existe uma carta $(U, h)$ em $p$ tal que

$$
(h(U \cap X), h(U \cap Y), h(p))
$$

satisfaz a condição $(\delta)$ no $\mathbb{R}^{n}$. Portanto, podemos assumir que $Y=\mathbb{R}^{d} \times O^{n-d}$ e $p=0$.

Se $\left.\left(\pi_{h}, \rho_{h}\right)\right|_{U^{\prime} \cap X}$ não é uma submersão para todo $U^{\prime} \subset U$, então podemos encontrar uma seqüência de pontos $\left\{m_{i}\right\}$ em $X$ convergindo para $p$, tal que, $\left.\left(\pi_{h}, \rho_{h}\right)\right|_{X}$ não é uma submersão em $m_{i}$. Mas, como $\left(\pi_{h}, \rho_{h}\right): \mathbb{R}^{n} \longrightarrow \mathbb{R}^{d} \times \mathbb{R}$ é uma submersão em $m_{i}$, e $\left.\left(\pi_{h}, \rho_{h}\right)\right|_{X}$ não o é, temos que $T_{m_{i}} X$ não é transversal ao núcleo da diferencial $\left(\pi_{h}, \rho_{h}\right)$ em $m_{i}$ (isto é, $T_{m_{i}} X$ é paralelo ao $\left.\operatorname{ker}\left(d\left(\pi_{h}, \rho_{h}\right)\left(m_{i}\right)\right)\right)$. Este núcleo é o complemento ortogonal de $\left(\mathbb{R}^{d} \times O\right)+\widehat{p m_{i}}$ em $\mathbb{R}^{n}$. De fato, sabemos que $\left(\left(\mathbb{R}^{d} \times O\right)+\widehat{p m_{i}}\right)^{\perp}=\left(\mathbb{R}^{d} \times O\right)^{\perp} \cap\left(\widehat{p m_{i}}\right)^{\perp}$. Além disso,

$$
d \rho_{h}(m) \in \operatorname{ker}\left(d \pi_{h}(m)\right)=\left(O \times \mathbb{R}^{n-d}\right) \Longleftrightarrow\left(\pi_{h}, \rho_{h}\right): U \longrightarrow \mathbb{R}^{d} \times \mathbb{R} \text { é uma submersão. }
$$

Note que, pela compacidade da Grasmanniana de subespaços vetoriais do $\mathbb{R}^{n}$, podemos supor que $T_{m_{i}} X \rightarrow \tau$ e que $\widehat{p m_{i}} \rightarrow l$.

Então, temos que $\tau$ não é transversal ao complemento ortogonal de $\left(\mathbb{R}^{d} \times O\right)+l$. Mas, isto contradiz a hipótese que $(X, Y)$ satisfaz as condições $(a)$ e $(\delta)$ em $p$; pois, pela condição $(a)$, obtemos $\left(\mathbb{R}^{d} \times O\right) \subset \tau$ e, por $(\delta)$, temos que $\sin \theta(l, \tau) \leq \delta_{0}<1$, o que implica que $l$ não é perpendicular a $\tau$. Logo, a projeção ortogonal em $\tau, \pi_{\tau}(l) \neq 0$.

Deste modo, segue-se que $\left(\mathbb{R}^{d} \times O\right)+\pi_{\tau}(l) \subset \tau$ e, consequentemente, o complemento ortogonal de $\left(\mathbb{R}^{d} \times l\right)$ é transversal a $\tau$.

Portanto, existem uma $C^{1}$-carta $(U, h)$ de $p$ para $Y$ em $M$, e uma vizinhança $U^{\prime}$ de $p, U^{\prime} \subset U$, tal que $\left.\left(\pi_{h}, \rho_{h}\right)\right|_{U^{\prime} \cap X}$ é uma submersão.

Corolário 3.6.1. Sejam $X, Y$ duas subvariedades regulares de $M$, com $Y \subset \bar{X}$, tal que o par $(X, Y)$ satisfaz as condições $(a)$ e $(\delta)$. Então, existe uma vizinhança tubular $T_{Y}$ de $Y$ em $M$, tal que, $\left.\left(\pi_{Y}, \rho_{Y}\right)\right|_{X}: X \cap T_{Y} \longrightarrow Y \times \mathbb{R}$ é uma submersão.

Lema 3.1. Sejam $V_{t}, W_{t}$ duas famílias de subespaços vetoriais em $\mathbb{R}^{n}$ tal que $\operatorname{dim} V_{t}+\operatorname{dim} W_{t} \geq n$ para todo t. Se para t próximo de zero, o ângulo entre $V_{t}$ e $W_{t}$ é limitado superiormente por uma constante $c>0$, isto é, $\theta\left(V_{t}, W_{t}\right)>c$, então 


$$
\lim _{t \rightarrow 0}\left(V_{t} \cap W_{t}\right)=\lim _{t \rightarrow 0} V_{t} \cap \lim _{t \rightarrow 0} W_{t} \text { (quando este limite existir). }
$$

(De fato é suficiente que $\lim _{t \rightarrow 0} V_{t}$ seja transversal ao $\lim _{t \rightarrow 0} W_{t}$ ).

Proposição 3.7. Todo espaço Whitney fraco estratificado é (c)-regular.

Demonstração: Seja $(A, S)$ um espaço Whitney fraco estratificado. Para provarmos que $(A, S)$ é $(c)$-regular é suficiente encontrar, para todo estrato $Y$ de $A$, uma aplicação de Thom $f_{Y}$, satisfazendo as condições para (c)-regularidade. Pela Proposição 3.6, a candidata a isto é a função tubular $\rho_{Y}$.

Sabemos que, $\rho_{Y}^{-1}(0)=Y$ e $\rho_{Y}: T_{Y} \cap X \longrightarrow \mathbb{R}_{+}$é uma submersão. Resta mostrarmos que $\rho_{Y}: \operatorname{Star}(Y) \longrightarrow\left(\mathbb{R}_{+}^{*},\{0\}\right)$ é uma aplicação de Thom.

Seja $X$ um estrato de $\operatorname{Star}(Y)$. Mostraremos que $\left.\left(\pi_{Y}, \rho_{Y}\right)\right|_{U \cap X}$ é uma submersão para toda retração local $\pi_{Y}: U \longrightarrow Y$ de classe $C^{1}$, provando que $T_{p} Y \subset \lim _{m \rightarrow p} \operatorname{ker}\left(\left.d \rho_{Y}\right|_{X}(m)\right)$, para todo $p \in Y$.

Tome $p_{0} \in Y$, para $m$ próximo de $p_{0}$ e $m \in X$. Pela condição $(\delta)$, segue-se a existência de um número real $c \in \mathbb{R}_{+}^{*}$, tal que o ângulo entre $T_{m} X$ e $T_{m} \rho_{Y}^{-1}\left(\rho_{Y}(m)\right)=\operatorname{ker}\left(d \rho_{Y}(m)\right)$ é limitado superiormente pela constante $c$ (suponhamos que $\operatorname{dim} X<n$, caso contrário ela é trivial).

Pelo lema 3.1, temos que:

$$
\begin{aligned}
\lim _{m \rightarrow p_{0}} T_{m} X \cap \lim _{m \rightarrow p_{0}} \operatorname{ker}\left(d \rho_{Y}(m)\right) & =\lim _{m \rightarrow p_{0}}\left(T_{m} X \cap \operatorname{ker}\left(d \rho_{Y}(m)\right)\right) \\
= & \lim _{m \rightarrow p_{0}} \operatorname{ker}\left(\left.d \rho_{Y}\right|_{X}(m)\right) .
\end{aligned}
$$

Resta, somente, provarmos que: $T_{p_{0}} Y \subset \lim _{m \rightarrow p_{0}} \operatorname{ker}\left(\left.d \rho_{Y}\right|_{X}(m)\right)$.

Mas, pela condição $(a): T_{p_{0}} Y \subset \lim _{m \rightarrow p_{0}} T_{m} X$.

Além disso, $T_{p_{0}} Y \subset \lim _{m \rightarrow p_{0}} T_{m} \rho_{Y}^{-1}\left(\rho_{Y}(m)\right)=\operatorname{ker}\left(\left.d \rho_{Y}\right|_{X}(m)\right)$ ( $\rho_{Y}$ é uma função tubular).

Portanto, $T_{p_{0}} Y \subset \lim _{m \rightarrow p_{0}} \operatorname{ker}\left(\left.d \rho_{Y}\right|_{X}(m)\right)$. 


\section{Capítulo 4}

\section{Comparando a (b)-regularidade de Whitney com o teste da razão de Kuo}

Nos capítulos anteriores apresentamos resultados garantindo que:

(I) A condição (b) de Whitney implica tivialidade topológica local (Teorema 2.4);

(II) Toda estratificação de Whitney é Whitney fraca (Exemplo 3.2). Entretanto, apresentamos um exemplo de uma estratificação Whitney fraca que não é (b)-regular (Exemplo 3.3). Portanto, a condição $(b)$ de Whitney é mais forte que a condição Whitney fraca;

(III) Todo espaço Whitney fraco é (c)-regular (Proposição 3.7). Além disso, o Exemplo 3.1, garante que a condição de regularidade $(b)$ de Whitney é mais forte que a condição de regularidade $(c)$ de K. Bekka. Portanto, os espaços com uma estratificação Whitney fraca formam uma classe intermediária entre a estratificação de Whitney e a estratificação (c)regular;

(IV) (c)-regularidade implica trivialidade topológica local (Observação 3.2 (2)).

No presente capítulo, introduziremos uma outra condição de regularidade conhecida por teste da razão de Kuo. Além disso, apresentaremos exemplos de estratificações em hipersuperfícies algébricas reais que satisfazem a condição $(b)$ de Whitney, mas falha o teste da razão de Kuo; logo, também falha a condição de regularidade $(w)$ de Verdier [24].

\subsection{Teste da Razão de Kuo}

Seja $V$ uma variedade analítica real (ou complexa). Faz-se a seguinte pergunta: Dada uma estratificação de $V=X_{1} \cup \ldots \cup X_{p}$, como podemos decidir se a estratificação é regular? Geralmente a condição de regularidade $(a)$ pode ser facilmente verificada, entretanto a condição $\left(b^{\prime}\right)$ nem sempre é fácil de ser verificada. Logo, em alguns casos fica difícil verificarmos se a estratificação é (b)-regular, ou seja, se a estratificação é regular. 
Nesta seção, introduziremos uma condição (por métodos analíticos) chamado o teste da razão, definida por Kuo em [11], que implica a condição (b) de Whitney.

Visto que variedades complexas em $\mathbb{C}^{n}$ podem ser consideradas como variedades reais em $\mathbb{R}^{2 n}$, consideraremos somente o caso real.

As condições de regularidade são de natureza local; logo, podemos restringir a seguinte situação no $\mathbb{R}^{n}$ :

Sejam $X$ um conjunto semianalítico e também uma variedade regular, e $Y$ uma variedade regular analítica tal que $0 \in Y \subset \bar{X} \backslash X$. Podemos considerar uma transformação analítica de $\mathbb{R}^{n}$ próximo de zero de modo que $Y$ coincida com o espaço tangente $T_{0} Y$ em 0 .

Agora, considere o conjunto $\widetilde{X} \subset \mathbb{R}^{n} \times \mathbb{R}^{n}$, contendo todos os pontos $(m, \nu(m))$, onde $m \in X$ e $\nu(m)$ é um vetor unitário normal de $X$ em $m$. Observe que $\widetilde{X}$ é um conjunto semianalítico (ver [28], §16).

Teorema 4.1. (Teste da Razão) Sejam X e Y como acima. Então, se para todo $\tau \in T_{0} Y$

$$
\lim _{m \rightarrow 0}\left(\frac{\left|\pi_{N_{m} X}(\tau)\right||\vec{m}|}{\left|\vec{m}-\pi_{Y}(\vec{m})\right|}\right)=0
$$

onde $\pi_{Y}$ denota a projeção ortogonal em $Y$, então $X$ é(b)-regular sobre $Y$ em 0.

Demonstração: Note que $\frac{|\vec{m}|}{\left|\vec{m}-\pi_{Y}(\vec{m})\right|} \geq 1$; logo, por $(r)$, temos que:

$$
\lim _{m \rightarrow 0}\left|\pi_{N_{m} X}(\tau)\right|=0, \text { para todo } \tau \in T_{0} Y \text {. }
$$

Então, a condição $(a)$ é satisfeita.

Agora, suponha que a condição $\left(b^{\prime}\right)$ não seja satisfeita. Então, existe $\varepsilon>0$ e pontos $(m, \nu(m)) \in \widetilde{X}$, onde $m$ está arbitrariamente próximo de 0 , satisfazendo:

$$
\nu(m) \cdot\left(\frac{\vec{m}-\pi_{Y}(\vec{m})}{\left|\vec{m}-\pi_{Y}(\vec{m})\right|}\right) \geq \varepsilon>0 .
$$

Assim, encontraremos uma contradição.

Excluindo-se o denominador na inequação (4.2), esta define um subconjunto semianalítico de $\widetilde{X}$. Logo, pelo Lema de seleção da curva, podemos escolher uma curva analítica $a(s), 0 \leq s \leq \delta$, com $a(0)=0$ e $a(s) \in X$, se $s \neq 0$, e vetores unitários normais $\nu(s) \in N_{a(s)} X$ para os quais (4.2) é satisfeita. (Aqui, $\nu(s)$ é analítico, mas não usaremos este fato).

Para simplificar, usaremos a seguinte notação:

- para um vetor $v \neq 0$, escreva $\mu(v)=\frac{v}{|v|}$, onde $|$.$| é a norma eclideana;$

- $\phi(s)$ denota a projeção ortogonal do vetor $\nu(s)$ num subespaço 2-dimensional gerado pelos vetores unitários $v_{1}(s)=\mu \frac{d a}{d s}$ e $v_{2}(s)=\mu\left(\pi_{Y} \frac{d a}{d s}\right)$.

(Substituíndo $\delta$ por um número menor, se necessário, podemos assumir que $\frac{d a}{d s} \notin T_{0} Y$ qualquer que seja $s$; então, $v_{1}(s)$ e $v_{2}(s)$ são linearmente independentes).

Escreva $v_{i}=v_{i}(s)$, por simplicidade. 
Note que $\nu(s) \cdot v_{1}=0$, visto que $\nu(s)$ é um vetor normal a $X$ em $a(s)$. Então,

$$
\begin{aligned}
\phi(s) & =\left\langle v_{1}, \nu(s)\right\rangle \cdot v_{1}+\left\langle\frac{v_{2}-\pi_{v_{1}}\left(v_{2}\right)}{\left|v_{2}-\pi_{v_{1}}\left(v_{2}\right)\right|}, \nu(s)\right\rangle \cdot\left(\frac{v_{2}-\pi_{v_{1}}\left(v_{2}\right)}{\left|v_{2}-\pi_{v_{1}}\left(v_{2}\right)\right|}\right) \\
& =\left(\frac{\nu(s) \cdot v_{2}}{\left|v_{2}-\pi_{v_{1}}\left(v_{2}\right)\right|}+\frac{\nu(s) \cdot \pi_{v_{1}}\left(v_{2}\right)}{\left|v_{2}-\pi_{v_{1}}\left(v_{2}\right)\right|}\right) \mu\left(v_{2}-\pi_{v_{1}}\left(v_{2}\right)\right) \\
& =\left(\frac{\nu(s) v_{2}}{\left|v_{2}-\pi_{v_{1}}\left(v_{2}\right)\right|}\right) \mu\left(v_{2}-\pi_{v_{1}}\left(v_{2}\right)\right)
\end{aligned}
$$

Assim,

$$
\phi(s)=\left(\frac{\nu(s) v_{2}}{\left|v_{2}-\pi_{v_{1}}\left(v_{2}\right)\right|}\right) \mu\left(v_{2}-\pi_{v_{1}}\left(v_{2}\right)\right),
$$

onde $\pi_{v}$ denota a projeção sobre a reta gerada por $v$.

$$
\text { Deste modo, segue que: } \begin{aligned}
|\phi(s)| & =\left|\left(\frac{\nu(s) v_{2}}{\left|v_{2}-\pi_{v_{1}}\left(v_{2}\right)\right|}\right) \mu\left(v_{2}-\pi_{v_{1}}\left(v_{2}\right)\right)\right| \\
& =\left|\frac{\nu(s) \cdot v_{2}}{\left|v_{2}-\pi_{v_{1}}\left(v_{2}\right)\right|}\right|\left|\frac{v_{2}-\pi_{v_{1}}\left(v_{2}\right)}{\left|v_{2}-\pi_{v_{1}}\left(v_{2}\right)\right|}\right| \\
& =\frac{\left|\nu(s) \cdot v_{2}\right|}{\left|v_{2}-\pi_{v_{1}}\left(v_{2}\right)\right|} \frac{\left|v_{2}-\pi_{v_{1}}\left(v_{2}\right)\right|}{\left|v_{2}-\pi_{v_{1}}\left(v_{2}\right)\right|} \\
& =\frac{\left|\nu(s) \cdot v_{2}\right|}{\left|v_{2}-\pi_{v_{1}}\left(v_{2}\right)\right|} .
\end{aligned}
$$

Como $v_{1}$ e $v_{2}$ são vetores unitários, temos que:

$$
\left|v_{1}-\pi_{v_{2}}\left(v_{1}\right)\right|=\left|v_{2}-\pi_{v_{1}}\left(v_{2}\right)\right|
$$

Logo,

$$
|\phi(s)|=\frac{\left|\nu(s) \cdot v_{2}\right|}{\left|v_{1}-\pi_{v_{2}}\left(v_{1}\right)\right|} .
$$

Agora, escreva $a(s)=(p(s), y(s))$, onde $y(s)$ está na componente conexa de $T_{0} Y$ e $p(s)$ está no complemento ortogonal de $Y$. Então, $\frac{d a}{d s}=\left(p^{\prime}(s), y^{\prime}(s)\right)$. Assim,

$$
v_{1}=\mu\left(\frac{d a}{d s}\right)=\mu\left(p^{\prime}(s), y^{\prime}(s)\right)=\frac{\left(p^{\prime}(s), y^{\prime}(s)\right)}{\left|\left(p^{\prime}(s), y^{\prime}(s)\right)\right|},
$$

e

$$
v_{2}=\mu\left(\pi_{Y}\left(\frac{d a}{d s}\right)\right)=\mu\left(0, y^{\prime}(s)\right)=\frac{\left(0, y^{\prime}(s)\right)}{\left|\left(0, y^{\prime}(s)\right)\right|}
$$

Então,

$$
\left|v_{1}-\pi_{v_{2}}\left(v_{1}\right)\right|=\left|\left(\frac{\left(p^{\prime}(s), y^{\prime}(s)\right)}{\left|\left(p^{\prime}(s), y^{\prime}(s)\right)\right|}\right)-\left\langle\frac{\frac{\left(0, y^{\prime}(s)\right)}{\left|\left(0, y^{\prime}(s)\right)\right|}}{\mid \frac{\left(0, y^{\prime}(s)\right)}{\left|\left(0, y^{\prime}(s)\right)\right|}}, \frac{\left(p^{\prime}(s), y^{\prime}(s)\right)}{\left|\left(p^{\prime}(s), y^{\prime}(s)\right)\right|}\right\rangle\right|
$$




$$
\begin{aligned}
& =\left|\left(\frac{\left(p^{\prime}(s), y^{\prime}(s)\right)}{\left|\left(p^{\prime}(s), y^{\prime}(s)\right)\right|}\right)-\left\langle\frac{\left(0, y^{\prime}(s)\right)}{\left|\left(0, y^{\prime}(s)\right)\right|}, \frac{\left(p^{\prime}(s), y^{\prime}(s)\right)}{\left|\left(p^{\prime}(s), y^{\prime}(s)\right)\right|}\right\rangle\right| \\
& =\left|\left(\frac{\left(p^{\prime}(s), y^{\prime}(s)\right)}{\left|\left(p^{\prime}(s), y^{\prime}(s)\right)\right|}\right)-\left(\frac{\left(0, y^{\prime}(s)\right)}{\left|\left(p^{\prime}(s), y^{\prime}(s)\right)\right|}\right)\right| \\
& =\left|\frac{\left(p^{\prime}(s), 0\right)}{\left|\left(p^{\prime}(s), y^{\prime}(s)\right)\right|}\right| .
\end{aligned}
$$

Logo,

$$
\left|v_{1}-\pi_{v_{2}}\left(v_{1}\right)\right|=\frac{\left|\left(\frac{d p}{d s}, 0\right)\right|}{\left|\frac{d a}{d s}\right|} .
$$

Mas, pela analiticidade,

$$
\left|s \frac{d a}{d s}\right| \sim|a(s)| \quad e \quad\left|s \frac{d p}{d s}\right| \sim|p(s)|
$$

Por outro lado, $\left|\nu(s) \cdot v_{2}\right| \leq\left|\pi_{N_{a(s)} X}\left(v_{2}\right)\right|$.

Disto, e por (4.5), (4.6) e (4.7), obtemos:

$$
|\phi(s)|=\frac{\left|\nu(s) \cdot v_{2}\right|}{\frac{\left|\left(\frac{d p}{d s}, 0\right)\right|}{\left|\frac{d a}{d s}\right|}}=\left|\nu(s) \cdot v_{2}\right| \frac{\left|\frac{d a}{d s}\right|}{\left|\left(\frac{d p}{d s}, 0\right)\right|} \leq \frac{\left|\pi_{N_{a(s)} X}\left(v_{2}\right)\right|\left|\frac{d a}{d s}\right|}{\left|\left(\frac{d p}{d s}, 0\right)\right|} \leq c\left(\frac{\left|\pi_{N_{a(s)} X}\left(v_{2}\right)\right||a(s)|}{|p(s)|}\right),
$$

onde $c$ é uma constante.

Assim, segue-se de $(r)$ que:

$$
\lim _{s \rightarrow 0}|\phi(s)|=0 .
$$

É claro que $\nu(s)-\phi(s)$ é perpendicular a $v_{1}$. Note, também, que $\nu(s)-\phi(s)$ é perpendicular a $v_{2}$, pois:

$$
\begin{aligned}
\left\langle\phi(s), v_{2}\right\rangle & =\frac{\left\langle\nu(s), v_{2}\right\rangle}{\left|v_{2}-\pi_{v_{1}}\left(v_{2}\right)\right|}\left\langle\mu\left(v_{2}-\pi_{v_{1}}\left(v_{2}\right)\right), v_{2}\right\rangle \\
& =\frac{\left\langle\nu(s), v_{2}\right\rangle}{\left|v_{2}-\pi_{v_{1}}\left(v_{2}\right)\right|}\left\langle\frac{v_{2}-\pi_{v_{1}}\left(v_{2}\right)}{\left|v_{2}-\pi_{v_{1}}\left(v_{2}\right)\right|}, v_{2}-\pi_{v_{1}}\left(v_{2}\right)\right\rangle \\
& =\left\langle\nu(s), v_{2}\right\rangle \frac{1}{\left|v_{2}-\pi_{v_{1}}\left(v_{2}\right)\right|^{2}}\left|v_{2}-\pi_{v_{1}}\left(v_{2}\right)\right|^{2} \\
& =\left\langle\nu(s), v_{2}\right\rangle .
\end{aligned}
$$

Deste modo,

$$
(\nu(s)-\phi(s)) \cdot \mu\left(v_{1}-\pi_{v_{2}}\left(v_{1}\right)\right)=0
$$

$\mathrm{ou}$

$$
(\nu(s)-\phi(s)) \cdot\left(\mu\left(\frac{d p}{d s}\right), 0\right)=0 .
$$


Por (4.8), $\lim _{s \rightarrow 0} \phi(s) \cdot\left(\mu\left(\frac{d p}{d s}\right), 0\right)=0$.

Logo, por (4.9) temos que:

$$
\lim _{s \rightarrow 0} \nu(s) \cdot\left(\mu\left(\frac{d p}{d s}\right), 0\right)=0 .
$$

Mas, pela analiticidade, $\lim _{s \rightarrow 0} \mu\left(\frac{d p}{d s}\right)=\lim _{s \rightarrow 0} \mu(p(s))$. Então, $\lim _{s \rightarrow 0} \nu(s) \cdot(\mu(p(s)), 0)=0$, o que contradiz (4.2).

Assim, a condição $\left(b^{\prime}\right)$ é satisfeita. Portanto, $X$ é $(b)$-regular sobre $Y$ em 0.

SIGNIFICADO GEOMÉTRICO DE $(r)$ : Primeiramente, note que:

$$
\lim _{m \rightarrow 0}\left(\frac{\left|\pi_{N_{m} X}(\tau)\right||\vec{m}|}{\left|\vec{m}-\pi_{Y}(\vec{m})\right|}\right)=\lim _{m \rightarrow 0}\left(\frac{\left|\pi_{N_{m} X}(\tau)\right|}{\mid \frac{\vec{m}-\pi_{Y}(\vec{m}) \mid}{|\vec{m}|}}\right),
$$

onde o numerador é a medida da distância entre $T_{m} X$ e $T_{0} Y$, enquanto o denominador pode ser interpretado como a distância do vetor unitário $\frac{\vec{m}}{|\vec{m}|}$ a $T_{0} Y$. Veja figura 4.1,

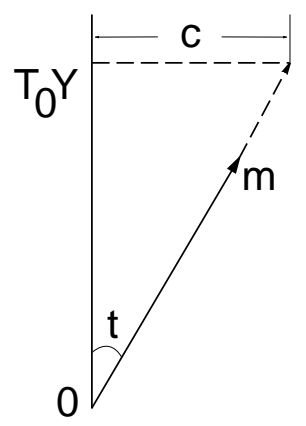

Figura 4.1: significado de $(r)$

onde $\frac{\left|\vec{m}-\pi_{Y}(\vec{m})\right|}{|\vec{m}|}=\sin t=c$, é a distância entre o vetor unitário $\frac{\vec{m}}{|\vec{m}|}$ e $T_{0} Y$. Logo, $(r)$ afirma que para algum $\tau \in T_{0} Y$, a razão dos dois valores acima tendem a zero quando $m \rightarrow 0$. Em outras palavras, a distância entre $T_{m} X$ e $T_{0} Y$ vai para zero tão rápido quanto $c$ vai para zero.

Observação 4.1. Em geral, a recíproca do Teorema 4.1 é falsa. Contudo, temos a recíproca parcial.

Teorema 4.2. Se $Y$ é 1-dimensional, então $(r)$ é uma condição necessária e suficiente para $X$ ser (b)-regular sobre $Y$ em 0 .

Demonstração: A prova é essencialmente o sentido inverso da prova do Teorema 4.1. Escolha $\tau \in T_{0} Y$ com $|\tau|=1$. Assumindo que $\operatorname{dim} Y=1$, segue-se que $\tau$ gera o espaço $T_{0} Y$ sobre $\mathbb{R}^{n}$.

Suponha que $X$ seja (b)-regular sobre $Y$ em 0 , mas que $(r)$ falhe. Assim, derivaremos uma contradição. 
Pelo Lema de seleção da curva, existe uma curva analítica $a(s), 0 \leq s \leq \delta$, com $a(0)=0$ e $a(s) \in X$ se $s \neq 0$, e vetores unitários $\tau(s) \in T_{0} Y$ e $\nu(s) \in N_{a(s)} X$ tais que:

$$
\frac{|\nu(s) \cdot \tau(s)||a(s)|}{|p(s)|} \geq \varepsilon
$$

onde $\varepsilon>0$ é uma constante.

Como anteriormente, $\phi(s)$ denota a projeção ortogonal de $\nu(s)$ sobre o espaço gerado pelos vetores

$$
v_{1}(s)=\mu\left(\frac{d a}{d s}\right) \quad \text { e } \quad v_{2}(s)=\tau(s)
$$

Por simplicidade, escreva $v_{i}(s)=v_{i}$ e $\tau(s)=\tau$. Então, por (4.3) e (4.4), temos que:

$$
\begin{aligned}
\phi(s) & =\frac{\nu(s) \cdot v_{2}}{\left|v_{1}-\pi_{v_{2}}\left(v_{1}\right)\right|} \mu\left(v_{2}-\pi_{v_{1}}\left(v_{2}\right)\right) \\
& =\frac{\nu(s) \cdot \tau}{\left|v_{1}-\pi_{\tau}\left(v_{1}\right)\right|} \mu\left(\tau-\pi_{v_{1}}(\tau)\right) .
\end{aligned}
$$

Assim, por um cálculo análogo ao feito na prova do Teorema 4.1 (em 4.9), obtemos:

$$
\begin{aligned}
(\nu(s)-\phi(s)) \cdot\left(\mu\left(\frac{d p}{d s}\right), 0\right)=0 & \Longrightarrow \nu(s) \cdot\left(\mu\left(\frac{d p}{d s}\right), 0\right)-\phi(s) \cdot\left(\mu\left(\frac{d p}{d s}\right), 0\right)=0 \\
& \Longrightarrow \nu(s) \cdot\left(\mu\left(\frac{d p}{d s}\right), 0\right)=\phi(s) \cdot\left(\mu\left(\frac{d p}{d s}\right), 0\right) \\
& \Longrightarrow \lim _{s \rightarrow 0} \nu(s) \cdot\left(\mu\left(\frac{d p}{d s}\right), 0\right)=\lim _{s \rightarrow 0} \phi(s) \cdot\left(\mu\left(\frac{d p}{d s}\right), 0\right)
\end{aligned}
$$

Mas, por (4.10) temos que: $\lim _{s \rightarrow 0} \nu(s) \cdot\left(\mu\left(\frac{d p}{d s}\right), 0\right)=0$. Logo, $\lim _{s \rightarrow 0} \phi(s) \cdot\left(\mu\left(\frac{d p}{d s}\right), 0\right)=0$.

Consequentemente,

$$
\lim _{s \rightarrow 0} \phi(s) \cdot \mu\left(v_{1}-\pi_{\tau}\left(v_{1}\right)\right)=0
$$

Agora, mostremos que

$$
\lim _{s \rightarrow 0} v_{1}(s) \in T_{0} Y .
$$

Suponha que (4.14) seja falsa, isto é, que $\lim _{s \rightarrow 0} v_{1}(s) \notin T_{0} Y$. Então:

$$
\lim _{s \rightarrow 0}\left|\frac{p(s)}{a(s)}\right| \neq 0 \quad \text { e } \quad \lim _{s \rightarrow 0}\left|\frac{a(s)}{p(s)}\right|<\infty .
$$

Pela condição $(a)$, temos que $\lim _{s \rightarrow 0} \nu(s) \cdot \tau=0$.

Deste modo, segue-se que $\lim _{s \rightarrow 0}\left(\frac{|\nu(s) \cdot \tau||a(s)|}{|p(s)|}\right)=0$, contradizendo (4.11).

Portanto, $\lim _{s \rightarrow 0} v_{1}(s) \in T_{0} Y$. 
Então, podemos assumir que $\lim _{s \rightarrow 0} v_{1}(s)=\tau$, já que $\operatorname{dim} Y=1$. Por um simples cálculo, obtemos:

$$
\lim _{s \rightarrow 0}\left[\mu\left(v_{1}-\pi_{\tau}\left(v_{1}\right)\right)+\mu\left(\tau-\pi_{v_{1}}(\tau)\right)\right]=0 .
$$

Logo, de (4.13) e (4.15) segue-se que: $\lim _{s \rightarrow 0} \phi(s) \cdot \mu\left(\tau-\pi_{v_{1}}(\tau)\right)=0$.

E, por (4.12) e (4.6):

$$
\lim _{s \rightarrow 0}|\phi(s)|=\lim _{s \rightarrow 0}\left(\frac{|\nu(s) \cdot \tau|\left|\frac{d a}{d s}\right|}{\left|\frac{d p}{d s}\right|}\right)=0 .
$$

Assim, por (4.7) temos que:

$$
\lim _{s \rightarrow 0}\left(\frac{|\nu(s) \cdot \tau||a(s)|}{|p(s)|}\right)=0, \text { contradizendo (4.11). Logo, }(r) \text { é satisfeita. }
$$

Portanto, $(r)$ também é uma condição necessária para $X$ ser $(b)$-regular sobre $Y$ em 0 .

\subsection{Exemplos}

Exemplo 4.1. (Ex.2.1, Capítulo 2) Considerando $f_{5}(x, y, z)=y^{2}-z^{2} x^{3}-x^{5}$, a variedade algébrica definida por $f_{5}$ é $V_{5}=\left\{(x, y, z) \in \mathbb{R}^{3} ; f_{5}(x, y, z)=0\right\}$.

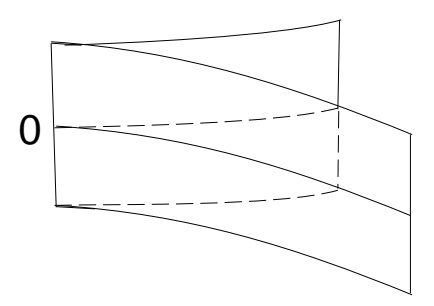

Figura 4.2: $V_{5}$

Sejam $Y=$ eixo $z$ e $X=V_{5} \backslash Y$. Já foi verificado anteriormente (exemplo 2.1, capítulo 2) que o par de estratos $(X, Y)$ é Whitney regular utilizando as condições de regularidade $(a)$ e $\left(b^{\prime}\right)$. A seguir, aplicaremos o teste da razão de Kuo para mostrarmos a Whitney regularidade em $(0,0,0)$.

Sabemos que, para todo $m=(x, y, z) \in X,(x, y) \neq(0,0)$, o espaço normal é gerado por:

$$
\nabla f_{5}(x, y, z)=\left(-3 x^{2} z^{2}-5 x^{4}, 2 y,-2 z x^{3}\right) .
$$

O vetor normal (direção) é:

$$
v(m)=\frac{\nabla f_{5}(x, y, z)}{\left\|\nabla f_{5}(x, y, z)\right\|}=\left(\frac{-3 x^{2} z^{2}-5 x^{4}}{\sqrt{A_{5}}}, \frac{2 y}{\sqrt{A_{5}}}, \frac{-2 z x^{3}}{\sqrt{A_{5}}}\right),
$$

onde: $A_{5}=\left(3 x^{2} z^{2}+5 x^{4}\right)^{2}+4 y^{2}+4 z^{2} x^{6}$.

Agora, seja $p=(0,0,0) \in Y$, então: 


$$
\overrightarrow{m p}=\vec{m}=(x, y, z) \Longrightarrow \pi_{Y}(\vec{m})=(0,0, z) \Longrightarrow \vec{m}-\pi_{Y}(\vec{m})=(x, y, 0)
$$

Logo, $\frac{\left|\pi_{N_{m} X}(\tau)\right|}{\frac{\left|\vec{m}-\pi_{Y}(\vec{m})\right|}{|\vec{m}|}}=\frac{|v(m) \cdot \tau||\vec{m}|}{\left|\vec{m}-\pi_{Y}(\vec{m})\right|}$

$$
\begin{aligned}
& =\frac{|v(m) \cdot \tau| \sqrt{x^{2}+y^{2}+z^{2}}}{\sqrt{x^{2}+y^{2}}} \\
& \leq \frac{|v(m) \cdot \tau|\left(\sqrt{x^{2}+y^{2}}+\sqrt{z^{2}}\right)}{\sqrt{x^{2}+y^{2}}} \\
& =\frac{|v(m) \cdot \tau| \sqrt{x^{2}+y^{2}}}{\sqrt{x^{2}+y^{2}}}+\frac{|v(m) \cdot \tau||z|}{\sqrt{x^{2}+y^{2}}} \\
& =|v(m) \cdot \tau|+\frac{|v(m) \cdot \tau||z|}{\sqrt{x^{2}+y^{2}}} .
\end{aligned}
$$

Do capítulo 2, temos que: $\lim _{m \rightarrow 0}|v(m) \cdot \tau|=0$.

Então, basta mostrarmos que $\lim _{m \rightarrow 0}\left(\frac{|v(m) \cdot \tau||z|}{\sqrt{x^{2}+y^{2}}}\right)=0$.

Note que, $\frac{|v(m) \cdot \tau||z|}{\sqrt{x^{2}+y^{2}}}=\frac{|v(m) \cdot \tau||z|}{\sqrt{x^{2}+z^{2} x^{3}+x^{5}}}$.

Além disso, para $|m|$ pequeno, $\sqrt{x^{2}+z^{2} x^{3}+x^{5}} \geq \frac{1}{2}|x|$ (pois os termos $z^{2} x^{3}$ e $x^{5}$ tem grau maior, logo vão para zero mais rápido).

$$
\begin{aligned}
& \text { Assim, } \lim _{m \rightarrow 0}\left(\frac{|v(m) \cdot \tau||z|}{\sqrt{x^{2}+y^{2}}}\right) \leq \lim _{m \rightarrow 0}\left(\frac{2|v(m) \cdot \tau||z|}{|x|}\right) \text {. } \\
& \text { Mas, } \quad \frac{2|v(m) \cdot \tau||z|}{|x|}=\frac{2|z|\left|2 z x^{3}\right|}{|x| \sqrt{\left(3 x^{2} z^{2}+5 x^{4}\right)^{2}+4 y^{2}+4 z^{2} x^{6}}} \\
& =\left|\frac{4 z^{2} x^{3}}{|x| \sqrt{\left(3 x^{2} z^{2}+5 x^{4}\right)^{2}+4 y^{2}+4 z^{2} x^{6}}}\right|
\end{aligned}
$$

Elevando ambos os membros ao quadrado, temos:

$$
\begin{aligned}
\left(\frac{2|v(m) \cdot \tau||z|}{|x|}\right)^{2} & =\left|\frac{16 z^{4} x^{6}}{x^{2}\left[\left(3 x^{2} z^{2}+5 x^{4}\right)^{2}+4 y^{2}+4 z^{2} x^{6}\right]}\right| \\
& \leq\left|\frac{16 z^{4} x^{6}}{4 y^{2} x^{2}}\right| \\
& =\left|\frac{4 z^{4} x^{4}}{x^{3}\left(z^{2}+x^{2}\right)}\right| \longrightarrow 0 \text { quando } x \rightarrow 0 .
\end{aligned}
$$


Consequentemente, $\lim _{m \rightarrow 0}\left(\frac{|v(m) \cdot \tau||z|}{\sqrt{x^{2}+y^{2}}}\right)=0$.

Logo, $\lim _{m \rightarrow 0}\left(|v(m) \cdot \tau|+\frac{|v(m) \cdot \tau||z|}{\sqrt{x^{2}+y^{2}}}\right)=0$.

Deste modo, segue-se que: $\lim _{m \rightarrow 0}\left(\frac{\left|\pi_{N_{m} X}(\tau)\right|}{\frac{\left|\vec{m}-\pi_{Y}(\vec{m})\right|}{|\vec{m}|}}\right)=\lim _{m \rightarrow 0}\left(\frac{\left|\pi_{N_{m} X}(\tau)\right||\vec{m}|}{\left|\vec{m}-\pi_{Y}(\vec{m})\right|}\right)=0$.

Portanto, pelo Teorema 4.1, $X$ é (b)-regular sobre $Y$ em 0 , ou seja, esta estratificação é Whitney regular.

Observação 4.2. O exemplo seguinte foi apresentado por S. Lojasiewicz (não publicado), o qual mostra que uma estratificação (b)-regular considerando os estratos como subvariedades do $\mathbb{R}^{n}$, pode não ter satisfeita nem a (a)-regularidade quando considerarmos os estratos em $\mathbb{C}^{n}$.

Seja $V^{*}$ uma variedade complexa em $\mathbb{C}^{3}$ definida pela mesma equação $f_{5}=0$, onde $f_{5}$ é dada no exemplo anterior. Sejam $Y^{*}=$ eixo complexo $z$ e $X^{*}$ o seu complemento em $V^{*}$. Então a condição de regularidade $(a)$ não é satisfeita em 0 , pois considerando o caminho $(x, 0, i x) \in V^{*}$, tem-se que o espaço normal a $X^{*}$ é gerado por:

$$
\nabla f_{5}(x, 0, i x)=\left(-2 x^{4}, 0,-2 i x^{4}\right)
$$

O vetor normal (direção) é:

$$
v(m)=\frac{\nabla f_{5}(x, 0, i x)}{\left\|\nabla f_{5}(x, 0, i x)\right\|}=\left(\frac{\left(-2 x^{4}, 0,-2 i x^{4}\right)}{\sqrt{\left|-2 x^{4}\right|^{2}+\left|-2 i x^{4}\right|^{2}}}\right)=\left(\frac{-2 x^{4}}{\sqrt{8 x^{8}}}, 0, \frac{-2 i x^{4}}{\sqrt{8 x^{8}}}\right) .
$$

Lembre-se que $\tau=(0,0,1)$ e, deste modo, segue-se que:

$$
|v(m) \cdot \tau|=\left|\left\langle\left(\frac{-2 x^{4}}{\sqrt{8 x^{8}}}, 0, \frac{-2 i x^{4}}{\sqrt{8 x^{8}}}\right),(0,0,1)\right\rangle\right|=\left|\frac{-2 i x^{4}}{\sqrt{8 x^{8}}}\right|
$$

Elevando ambos os membros ao quadrado, temos:

$$
|v(m) \cdot \tau|^{2}=\left|\frac{4 x^{8}}{8 x^{8}}\right|=\frac{1}{2}
$$

Logo, $\lim _{m \rightarrow 0}\left|\pi_{N_{m} X^{*}}(\tau)\right|=\lim _{m \rightarrow 0}|v(m) \cdot \tau|=\lim _{m \rightarrow 0} \frac{1}{\sqrt{2}}=\frac{\sqrt{2}}{2} \neq 0$.

Portanto, $X^{*}$ não é $(a)$-regular sobre $Y^{*}$ em 0.

Observação 4.3. Podemos generalizar o exemplo 4.1 para a variedade real $V(k, l, m)$ definida por $y^{2 k}-z^{2} x^{2(k+l)+1}-x^{2(k+l+m)+1}=0 \mathrm{em} \mathbb{R}^{3}$, onde $k>0, l \geq 0, m \geq 0$ são inteiros. Basta considerarmos a seguinte estratificação: $Y=$ eixo $z$ e $X$ o seu complemento em $V$. Então, um cálculo similiar ao do exemplo 4.1 mostra que $X$ é Whitney regular sobre $Y$. 
O próximo exemplo mostra que o teste da razão de Kuo falha para estratificações não analíticas.

Exemplo 4.2. Considere a "espiral lenta" $V_{6}=\left\{\left(e^{-\theta}, \theta\right) ; \theta \in \mathbb{R}\right\} \cup\{(0,0)\}$ e a estratificação dada por $Y=\{(0,0)\}$ e $X$ o complemento de $Y$ em $V_{6}$.

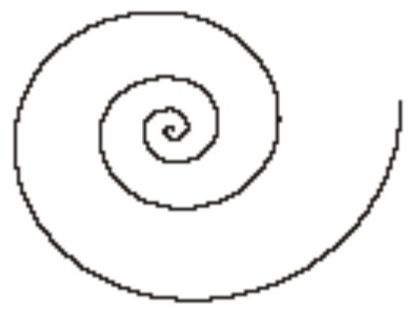

Figura 4.3: $V_{6}$

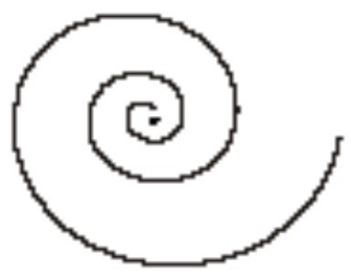

Figura 4.4: Estrat.1

É fácil ver que, neste caso, o teste da razão é satisfeito, mas a estratificação não é (b)-regular. De fato, note que o estrato $X$ pode ser parametrizado por:

$$
\gamma(\theta)=e^{-\theta}(\sin \theta, \cos \theta)
$$

Assim, $\gamma^{\prime}(\theta)=e^{-\theta}((\cos \theta,-\sin \theta)-(\sin \theta, \cos \theta))$. Um simples cálculo mostra que o teste da razão vale. Entretanto, para $\theta_{k}=\frac{\pi}{4}+2 k \pi$, tem-se:

$$
\frac{\gamma^{\prime}\left(\theta_{k}\right)}{\left\|\gamma^{\prime}\left(\theta_{k}\right)\right\|}=(0,-1) \quad \text { e } \quad \gamma\left(\theta_{k}\right)=\frac{1}{2} e^{-\theta_{k}}(\sqrt{2}, \sqrt{2}) .
$$

Agora, a seqüência de pontos $\gamma\left(\theta_{k}\right)$ converge para a origem 0 quando $k \rightarrow \infty$, a seqüência de espaços tangentes para o subespaço gerado por $(0,1)$ e, finalmente, a seqüência de secantes $\widehat{\gamma\left(\theta_{k}\right) 0}$ converge para a reta gerada por $(1,1)$ passando pela origem. Logo, a "espiral lenta" não pode satisfazer a condição $(b)$ de Whitney.

Além disso, este argumento também mostra que não existe nenhuma decomposição que torna a "espiral lenta" um espaço Whitney estratificado.

\subsection{A (w)-regularidade de Verdier}

Seja $X$ uma subvariedade de classe $C^{1}$ e um conjunto subanalítico do $\mathbb{R}^{n}$. Seja $Y$ uma subvariedade regular analítica do $\mathbb{R}^{n}$ tal que $0 \in Y \subset \bar{X} \backslash X$.

Definição 4.1. Dizemos que $X$ é $(w)$-regular sobre $Y$ no ponto 0 se existem uma constante $c>0$ e uma vizinhança $U_{0}$ de 0 em $\mathbb{R}^{n}$ tais que:

$$
d\left(T_{p} Y, T_{m} X\right) \leq c|m-p|, \quad(w)
$$

para todo $m \in U_{0} \cap X$ e todo $p \in U_{0} \cap Y$.

Aqui, $d(.,$.$) é definida como se segue:$ 
Definição 4.2. Sejam $A, B$ subespaços vetoriais do $\mathbb{R}^{n}$, então:

$$
d(A, B)=\sup _{|a|=1}\left|a-\pi_{B}(a)\right| \text {, onde } a \in A \text { e } \pi_{B} \text { é a projeção ortogonal sobre } B .
$$

Note que, $d(A, B)=0 \Longleftrightarrow A \subset B$.

Observação 4.4. Decorre da definição que a condição $(w)$ é $C^{2}$-invariante, ou mais precisamente, que é invariante sobre um $C^{1}$-difeomorfismo com derivada Lipschitz. Veremos abaixo que $(w)$ não é $C^{1}$-invariante.

Suponhamos que $Y$ seja linear (caso contrário, aplicamos um isomorfismo analítico local próximo de $0 \mathrm{em} \mathbb{R}^{n}$ ). Seja $\pi_{Y}$ a projeção ortogonal sobre $Y$.

Reformule $(w)$ pela condição que $\left(\frac{d\left(T_{p} Y, T_{m} X\right)}{|m-p|}\right)$ é limitada próximo de zero. Então, em particular, $\left(\frac{d\left(T_{0} Y, T_{m} X\right)}{\left|\vec{m}-\pi_{Y}(\vec{m})\right|}\right)$ é limitada para $m$ próximo de zero (lembre-se que $Y$ é linear).

Note que, na definição de $(r)$, podemos substituir $\left|\pi_{N_{m} X}(\tau)\right|$ por $d\left(\langle\tau\rangle, T_{m} X\right)$, onde $\tau \in T_{0} Y$ é um vetor unitário. Logo, está claro que, se $X$ é $(w)$-regular sobre $Y$ em 0 , então $(X, Y)$ satisfaz o teste da razão de Kuo em 0.

Na seção 4.1 ficou provado que $(r) \Rightarrow(b)$-regularidade e que $(b) \Leftrightarrow(r)$ quando o estrato de menor dimensão for 1-dimensional. Então, surge a seguinte pergunta:

O que acontecerá quando este estrato for ao menos 2-dimensional? Aqui, mostraremos que $(b) \nRightarrow(r)$ neste caso.

Observação 4.5. Se $(b)$ (respectivamente $(w)$ ) vale para um par de estratos $(X, Y)$ no ponto 0 em $\mathbb{R}^{n}$, então $(b)$ (respectivamente $(w)$ ) vale para $(X \times \mathbb{R}, Y \times \mathbb{R})$ ao longo de $0 \times \mathbb{R}$ em $\mathbb{R}^{n} \times \mathbb{R}$. Entretanto, veremos a seguir que $(r)$ não tem esta propriedade.

Proposição 4.1. Seja $(X, Y)$ um par de estratos no $\mathbb{R}^{n}$, o qual não é (w)-regular em 0 (mas, possivelmente satisfazendo $(r))$, e seja $Y$ linear. Então $(X \times \mathbb{R}, Y \times \mathbb{R})$ não satisfaz $(r)$ em qualquer ponto de $0 \times \mathbb{R}$ em $\mathbb{R}^{n} \times \mathbb{R}$.

Demonstração: Sejam $X, Y$ de dimensões $x, y$ respectivamente, e identifique o conjunto de subespaços 1-dimensionais $T_{0} Y$ com a variedade Grasmanniana $G(1, y)$.

Defina três subconjuntos de $\mathbb{R}^{n} \times \mathbb{R}^{n} \times G(x, n) \times G(1, y) \times \mathbb{R}$ :

$V_{1}=\left\{\left(m, \pi_{Y}(m), T_{m} X\right) ; m \in X\right\} \times G(1, y) \times \mathbb{R}$

$V_{2}=\{(m, p, T,\langle\tau\rangle, \varepsilon) ;|m-p|<\varepsilon d(\langle\tau\rangle, T)\}$

$V_{3}=\mathbb{R}^{n} \times \mathbb{R}^{n} \times\left\{(T,\langle\tau\rangle) ; d(\langle\tau\rangle, T)=d\left(T_{0} Y, T\right)\right\} \times \mathbb{R}$.

Assim, $V_{1}$ é subanalítico (usando [24], Lema 1.6), $V_{2}$ é semialgébrico e $V_{3}$ é algébrico. Logo, $V=V_{1} \cap V_{2} \cap V_{3}$ é um conjunto subanalítico.

Temos que $(w)$ falha para o par de estratos $(X, Y)$ em 0 , o que é equivalente à existência de um plano $v \in G(x, n)$ e $\tau \in T_{0} Y \operatorname{com}\|\tau\|=1$, tal que:

$$
(0,0, v,\langle\tau\rangle, 0) \in \bar{V} \subset \mathbb{R}^{n} \times \mathbb{R}^{n} \times G(x, n) \times G(1, y) \times \mathbb{R} .
$$


Pelo Lema de seleção da curva podemos encontrar uma curva analítica $a:[0,1] \longrightarrow \mathbb{R}^{n} \times \mathbb{R}^{n} \times G(x, n) \times G(1, y) \times \mathbb{R}$, tal que $a(0)=(0,0, v,\langle\tau\rangle, 0)$ e $a(s) \in V$, se $s \neq 0$.

Agora, escreva $a(s)=\left(m_{s}, \pi_{Y}\left(m_{s}\right), T_{m_{s}} X,\left\langle\tau_{s}\right\rangle, \varepsilon_{s}\right)$, onde $\tau_{s} \in T_{0} Y,\left\|\tau_{s}\right\|=1$, e $\tau_{s} \rightarrow \tau$ quando $s \rightarrow 0$.

Então, $\frac{d\left(\left\langle\tau_{s}\right\rangle, T_{m_{s}} X\right)}{\left|m_{s}-\pi_{Y}\left(m_{s}\right)\right|}$ é ilimitada quando $s \rightarrow 0$.

Afirmamos que $d\left(\langle\tau\rangle, T_{m_{s}} X\right) \geq \frac{1}{2} d\left(\left\langle\tau_{s}\right\rangle, T_{m_{s}} X\right)$, para $s$ suficientemente pequeno. Isto é uma conseqüência da definição de $V_{3}$ como segue:

Seja $\varphi_{s}$ o ângulo positivo entre $\tau$ e $\tau_{s}$, podemos assumir que $0 \leq \varphi_{s} \leq \frac{\pi}{2}$, então:

$$
\tau=\tau_{s} \cos \varphi_{s}+u_{s} \sin \varphi_{s}, \text { onde }\left\|u_{s}\right\|=1, \tau_{s} \perp u_{s}
$$

De fato, seja $w_{s}=\tau-\left\langle\tau, \tau_{s}\right\rangle \cdot \tau_{s}=\tau-\cos \varphi_{s} \cdot \tau_{s}$. Agora, tome $u_{s}=\frac{w_{s}}{\left\|w_{s}\right\|}$, então $\left\|u_{s}\right\|=1 \mathrm{e}$ $\tau_{s} \perp u_{s}$.

Note que, $\left\|w_{s}\right\|^{2}=1-2 \cos ^{2} \varphi_{s}+\cos ^{2} \varphi_{s}=1-\cos ^{2} \varphi_{s}=\sin ^{2} \varphi_{s}$.

Logo, $\left\|w_{s}\right\|=\sin \varphi_{s}$, já que $0 \leq \varphi_{s} \leq \frac{\pi}{2}$. Então, $u_{s}=\frac{w_{s}}{\sin \varphi_{s}}$.

Deste modo, segue-se que

$\tau_{s} \cos \varphi_{s}+u_{s} \sin \varphi_{s}=\tau_{s} \cos \varphi_{s}+\frac{w_{s}}{\sin \varphi_{s}} \sin \varphi_{s}=\tau_{s} \cos \varphi_{s}+w_{s}=\tau_{s} \cos \varphi_{s}+\tau-\tau_{s} \cos \varphi_{s}=\tau$.

Agora, seja $\pi_{s}$ a projeção ortogonal sobre $T_{m_{s}} X$.

Assim, $\quad d\left(\langle\tau\rangle, T_{m_{s}} X\right)=\left|\tau-\pi_{s}(\tau)\right|$

$$
\begin{aligned}
& =\left|\left(\tau_{s} \cos \varphi_{s}+u_{s} \sin \varphi_{s}\right)-\left(\cos \varphi_{s} \pi_{s}\left(\tau_{s}\right)+\sin \varphi_{s} \pi_{s}\left(u_{s}\right)\right)\right| \\
& =\left|\left(\tau_{s}-\pi_{s}\left(\tau_{s}\right)\right) \cos \varphi_{s}+\left(u_{s}-\pi_{s}\left(u_{s}\right)\right) \sin \varphi_{s}\right| \\
& \geq\left|\tau_{s}-\pi_{s}\left(\tau_{s}\right)\right| \cos \varphi_{s}-\left|u_{s}-\pi_{s}\left(u_{s}\right)\right| \sin \varphi_{s} .
\end{aligned}
$$

Mas, pela definição de $V_{3}$, temos que:

$$
\left|\tau_{s}-\pi_{s}\left(\tau_{s}\right)\right| \geq\left|u_{s}-\pi_{s}\left(u_{s}\right)\right| \Rightarrow-\left|u_{s}-\pi_{s}\left(u_{s}\right)\right| \geq-\left|\tau_{s}-\pi_{s}\left(\tau_{s}\right)\right| .
$$

Logo,

$\left|\tau_{s}-\pi_{s}\left(\tau_{s}\right)\right| \cos \varphi_{s}-\left|u_{s}-\pi_{s}\left(u_{s}\right)\right| \sin \varphi_{s} \geq\left|\tau_{s}-\pi_{s}\left(\tau_{s}\right)\right| \cos \varphi_{s}-\left|\tau_{s}-\pi_{s}\left(\tau_{s}\right)\right| \sin \varphi_{s}$

$$
\begin{aligned}
& \geq\left|\tau_{s}-\pi_{s}\left(\tau_{s}\right)\right|\left(\cos \varphi_{s}-\sin \varphi_{s}\right) \\
& \geq d\left(\left\langle\tau_{s}\right\rangle, T_{m_{s}} X\right)\left(\cos \varphi_{s}-\sin \varphi_{s}\right) .
\end{aligned}
$$

Então, $d\left(\langle\tau\rangle, T_{m_{s}} X\right) \geq d\left(\left\langle\tau_{s}\right\rangle, T_{m_{s}} X\right)\left(\cos \varphi_{s}-\sin \varphi_{s}\right)$.

Como $\tau_{s} \rightarrow 0$ quando $s \rightarrow 0$, para $s$ suficientemente pequeno, segue-se que 


$$
d\left(\langle\tau\rangle, T_{m_{s}} X\right) \geq \frac{1}{2} d\left(\left\langle\tau_{s}\right\rangle, T_{m_{s}} X\right) .
$$

Assim, deduzimos que $\frac{d\left(\langle\tau\rangle, T_{m_{s}} X\right)}{\left|m_{s}-\pi_{Y}\left(m_{s}\right)\right|}$ também é ilimitado quando $s \rightarrow 0$.

Além disso, da definição de $V_{2}$, obtemos:

$$
\frac{1}{2}\left(\frac{d\left(\left\langle\tau_{s}\right\rangle, T_{m_{s}} X\right)}{\left|m_{s}-\pi_{Y}\left(m_{s}\right)\right|}\right)>\frac{1}{2} \varepsilon^{-1} \sim s^{-k}, \text { para algum } k \geq 1 .
$$

Logo, $\frac{d\left(\langle\tau\rangle, T_{m_{s}} X\right)}{\left|m_{s}-\pi_{Y}\left(m_{s}\right)\right|} \sim s^{-k}$, para algum $k \geq 1$.

Em $\mathbb{R}^{n} \times \mathbb{R}$, considere a curva $q(s)=\left(m_{s}, s_{0}+s\right)$.

Usando a inclusão canônica $T_{0} Y \subset T_{\left(0, s_{0}\right)}(Y \times \mathbb{R})$, podemos considerar $\tau$ como um vetor unitário de $T_{\left(0, s_{0}\right)}(Y \times \mathbb{R})$.

$$
\begin{aligned}
\text { Então, } \quad \frac{d\left(\langle\tau\rangle, T_{q(s)}(X \times \mathbb{R})\right) \cdot\left|q(s)-\left(0, s_{0}\right)\right|}{\left|q(s)-\pi_{Y \times \mathbb{R}}(q(s))\right|} & =\frac{d\left(\langle\tau\rangle, T_{m_{s}} X\right) \cdot\left|\left(m_{s}, s\right)\right|}{\left|m_{s}-\pi_{Y}\left(m_{s}\right)\right|} \\
& \geq \frac{d\left(\langle\tau\rangle, T_{m_{s}} X\right) \cdot s}{\left|m_{s}-\pi_{Y}\left(m_{s}\right)\right|} \\
& \sim s^{-k} \cdot s=s^{-(k-1)} .
\end{aligned}
$$

Assim, $\frac{d\left(\langle\tau\rangle, T_{q(s)}(X \times \mathbb{R})\right) \cdot\left|q(s)-\left(0, s_{0}\right)\right|}{\left|q(s)-\pi_{Y \times \mathbb{R}}(q(s))\right|} \sim s^{-(k-1)} \nmid 0$ quando $s \rightarrow 0$, já que $k \geq 1$.

Logo, o teste da razão de Kuo falha para o par $(X \times \mathbb{R}, Y \times \mathbb{R})$ em qualquer ponto $\left(0, s_{0}\right)$ de $0 \times \mathbb{R}$ em $\mathbb{R}^{n} \times \mathbb{R}$, completando a prova.

Exemplo 4.3. Seja $V_{1}=\left\{y^{3}=z^{2} x^{3}+x^{5}\right\} \subset \mathbb{R}^{3}$, e sejam $Y=$ eixo $z$ e $X$ o complementar de $Y$ em $V_{1}$. Considere $f_{1}(x, y, z)=-y^{3}+z^{2} x^{3}+x^{5}$, então para $m=(x, y, z) \in X,(x, y) \neq(0,0)$, temos que o espaço normal é gerado por:

$$
\nabla f_{1}(x, y, z)=\left(5 x^{4}+3 x^{2} z^{2},-3 y^{2}, 2 z x^{3}\right) .
$$

O vetor normal (direção) é:

$$
v(m)=\frac{\nabla f_{1}(x, y, z)}{\left\|\nabla f_{1}(x, y, z)\right\|}=\left(\frac{5 x^{4}+3 x^{2} z^{2}}{\sqrt{C_{1}}}, \frac{-3 y^{2}}{\sqrt{C_{1}}}, \frac{2 z x^{3}}{\sqrt{C_{1}}}\right),
$$

onde $C_{1}=\left(5 x^{4}+3 x^{2} z^{2}\right)^{2}+9 y^{4}+4 z^{2} x^{6}$.

Além disso, se $\tau \in T_{p} Y$, podemos assumir que $\tau=(0,0,1)$, visto que $T_{p} Y$ é 1-dimensional.

Logo,

$$
\left|\pi_{N_{m} X}(\tau)\right|=|v(m) \cdot \tau|=\left|\frac{2 z x^{3}}{\sqrt{\left(5 x^{4}+3 x^{2} z^{2}\right)^{2}+9 y^{4}+4 z^{2} x^{6}}}\right| .
$$


Elevando ambos os membros ao quadrado, temos que:

$$
\left|\pi_{N_{m} X}(\tau)\right|^{2}=\left|\frac{4 z^{2} x^{6}}{\left(5 x^{4}+3 x^{2} z^{2}\right)^{2}+9 y^{4}+4 z^{2} x^{6}}\right|=\leq\left|\frac{4 z^{2} x^{6}}{9 y^{4}}\right|=\left|\frac{4 z^{2} x^{6}}{9\left(z^{2} x^{3}+x^{5}\right)^{\frac{4}{3}}}\right| .
$$

Elevando ao cubo, obtemos:

$$
\left|\pi_{N_{m} X}(\tau)\right|^{6}=\left|\frac{64 z^{6} x^{18}}{729\left(z^{2} x^{3}+x^{5}\right)^{4}}\right|=\left|\frac{64 z^{6} x^{6}}{729\left(z^{2}+x^{2}\right)^{4}}\right| .
$$

Vamos dividir em 2 casos:

Caso I: $p=\left(0,0, z^{\prime}\right)$, com $z^{\prime} \neq 0$. Aqui, é claro que $\lim _{m \rightarrow p}\left|\frac{64 z^{6} x^{6}}{729\left(z^{2}+x^{2}\right)^{4}}\right|=0$.

Caso II: $p=(0,0,0)$. Aqui, podemos encontrar uma curva analítica $\alpha(t)=\left(x_{0} t^{n}+\ldots, y_{0} t^{m}+\ldots, z_{0} t^{p}+\ldots\right), \operatorname{com} \alpha(0)=0$ e $\alpha(t) \in V_{1}$ se $t \neq 0$, tal que:

$$
\lim _{m \rightarrow p}\left|\frac{64 z_{0}^{6} x_{0}^{6} t^{6 p+6 n}+\ldots}{729\left(z_{0}^{2} t^{2 p}+x_{0}^{2} t^{2 n}\right)^{4}+\ldots}\right|=0
$$

e consequentemente,

$$
\lim _{m \rightarrow p}\left|\frac{64 z^{6} x^{6}}{729\left(z^{2}+x^{2}\right)^{4}}\right|=0
$$

Deste modo, segue-se que $\lim _{m \rightarrow 0}\left|\pi_{N_{m} X}(\tau)\right|=0$. Portanto, $X$ é $(a)$-regular sobre $Y$.

Agora, considere $p=\left(0,0, z_{0}\right) \in Y$, então:

$$
\overrightarrow{m p}=\left(x, y, z-z_{0}\right) \Longrightarrow \pi_{p}(\overrightarrow{m p})=\left(0,0, z-z_{0}\right) \Longrightarrow \overrightarrow{m p}-\pi_{p}(\overrightarrow{m p})=(x, y, 0)
$$

Consequentemente, $\frac{\overrightarrow{m p}-\pi_{p}(\overrightarrow{m p})}{\left|\overrightarrow{m p}-\pi_{p}(\overrightarrow{m p})\right|}=\frac{(x, y, 0)}{|(x, y, 0)|}=\frac{(x, y, 0)}{\sqrt{x^{2}+y^{2}}}$.

Logo,

$$
\begin{aligned}
\left|\pi_{N_{m} X}\left(\frac{\vec{m}-\pi_{p}(\vec{m})}{\left|\vec{m}-\pi_{p}(\vec{m})\right|}\right)\right| & =\left|\left\langle v(m), \frac{(x, y, 0)}{\sqrt{x^{2}+y^{2}}}\right\rangle\right| \\
& =\left|\frac{5 x^{5}+3 x^{3} z^{2}-3 y^{3}}{\sqrt{\left(5 x^{4}+3 x^{2} z^{2}\right)^{2}+9 y^{4}+4 z^{2} x^{6}} \sqrt{x^{2}+y^{2}}}\right| \\
& =\left|\frac{5 x^{5}+3 x^{3} z^{2}-3\left(x^{5}+x^{3} z^{2}\right)}{\left[\left(5 x^{4}+3 x^{2} z^{2}\right)^{2}+9 y^{4}+4 z^{2} x^{6}\right]^{\frac{1}{2}}\left[x^{2}+y^{2}\right]^{\frac{1}{2}}}\right| \\
(\star) & =\left|\frac{2 x^{5}}{\left[\left(5 x^{4}+3 x^{2} z^{2}\right)^{2}+9 y^{4}+4 z^{2} x^{6}\right]^{\frac{1}{2}}\left[x^{2}+y^{2}\right]^{\frac{1}{2}}}\right| .
\end{aligned}
$$

Afirmamos que $\lim _{m \rightarrow 0}(\star)=0$. De fato, pelo Lema de seleção da curva, existe uma curva analítica $\beta(s)=\left(x_{0} s^{n}+\ldots, y_{0} s^{m}+\ldots, z_{0} s^{p}+\ldots\right)$, com $\beta(0)=0$ e $\beta(s) \in V_{1}$, se $s \neq 0$. Vamos verificar que $\lim _{s \rightarrow 0}(\star) \circ \beta(s)=0$. 
Primeiramente, note que

$$
y_{0}^{3} s^{3 m}+\ldots=z_{0}^{2} x_{0}^{3} s^{2 p+3 n}+x_{0}^{5} s^{5 n}+\ldots
$$

Além disso, considere:

$$
g\left(x_{0}, y_{0}, z_{0}\right)=\left(5 x_{0}^{4} s^{4 n}+3 x_{0}^{2} z_{0}^{2} s^{2 n+2 p}\right)^{2}+9 y_{0}^{4} s^{4 m}+4 x_{0}^{6} z_{0}^{2} s^{6 n+2 p}+\ldots
$$

Agora, basta considerarmos os seguintes casos:

Caso I: $n<p \Rightarrow 3 m=5 n \Leftrightarrow m=\frac{5}{3} n$.

Caso II: $n=p \Rightarrow 3 m=5 n \Leftrightarrow m=\frac{5}{3} n$.

Caso III: $n>p \Rightarrow 3 m=2 p+3 n$.

Nos 3 casos, em ( $(\star)$, obtemos:

$\left|\frac{2 x_{0} s^{5 n}}{\left[g\left(x_{0}, y_{0}, z_{0}\right)\right]^{\frac{1}{2}}\left(x_{0}^{2} s^{2 n}+y_{0}^{2} s^{2 m}+\ldots\right)^{\frac{1}{2}}}\right| \sim \frac{s^{5 n}}{s^{2 m} s^{n}} \sim \frac{s^{4 n}}{s^{2 m}} \longrightarrow 0$ quando $s \rightarrow 0$, pois $2 m<4 n$ em ambos os casos.

Deste modo, segue-se que: $\lim _{m \rightarrow p}\left|\pi_{N_{m} X}\left(\frac{\overrightarrow{m p}-\pi_{p}(\overrightarrow{m p})}{\left|\overrightarrow{m p}-\pi_{p}(\overrightarrow{m p})\right|}\right)\right|=0$ e, consequentemente, $X$ é $\left(b^{\prime}\right)$-regular sobre $Y$.

Portanto, $(b)$ vale para $(X, Y)$. Logo, pelo Teorema 4.2, deduzimos que $(X, Y)$ satisfaz $(r)$ em 0 , visto que $\operatorname{dim} Y=1$.

Considere a curva $p(s)=\left(s^{3}, \sqrt[3]{2} \cdot s^{5}, s^{3}\right) \in V_{1}$. Lembre-se que a direção normal a $X$ em $(x, y, z)$ é:

$$
\nabla f_{1}(x, y, z)=\left(3 x^{2} z^{2}+5 x^{4}:-3 y^{2}: 2 z x^{3}\right)
$$

$\operatorname{Em} p(s)$ isto se torna:

$$
\left(3 s^{6} s^{6}+5 s^{12}:-3\left(\sqrt[3]{2} s^{5}\right)^{2}: 2 s^{9} s^{3}\right)=\left(8 s^{2}:-3.2^{\frac{2}{3}}: 2 s^{2}\right) .
$$

Portanto, $\quad d\left(T_{0} Y, T_{p(s)} X\right)=\left\langle\frac{\nabla f_{1}\left(s^{3}, \sqrt[3]{2} \cdot s^{5}, s^{3}\right)}{\left\|\nabla f_{1}\left(s^{3}, \sqrt[3]{2} \cdot s^{5}, s^{3}\right)\right\|},(0,0,1)\right\rangle$

$$
\begin{aligned}
& =\frac{2 s^{2}}{\sqrt{\left(8 s^{2}\right)^{2}+\left(-3.2^{\frac{2}{3}}\right)^{2}+\left(2 s^{2}\right)^{2}}} \\
& =\frac{2 s^{2}}{\sqrt{64 s^{4}+9 \sqrt[3]{2^{4}}+4 s^{4}}} \\
& =\frac{2 s^{2}}{\left(68 s^{4}+18 \sqrt[3]{2}\right)^{\frac{1}{2}}} \sim 1 .
\end{aligned}
$$

Além disso, $\quad\left|p(s)-\pi_{Y}(p(s))\right|=\left|\left(s^{3}, \sqrt[3]{2} \cdot s^{5}, s^{3}\right)-\left(0,0, s^{3}\right)\right|$

$$
\begin{aligned}
& =\sqrt{s^{6}+\left(\sqrt[3]{2} \cdot s^{5}\right)^{2}} \\
& =s^{3}\left(1+2^{\frac{2}{3}} s^{4}\right)^{\frac{1}{2}} \sim s^{3} .
\end{aligned}
$$


Então, $\frac{d\left(T_{0} Y, T_{p(s)} X\right)}{\left|p(s)-\pi_{Y}(p(s))\right|} \sim \frac{1}{s^{3}}$ que é ilimitado quando $s \rightarrow 0$.

Portanto, $(w)$ falha para $(X, Y)$ em 0 .

Considere $V_{1}^{\prime}=V_{1} \times \mathbb{R} \subset \mathbb{R}^{4}=\{(x, y, z, u)\}$, e sejam $Y^{\prime}=Y \times \mathbb{R}=\{y=x=0\} \subset \mathbb{R}^{4}$ e $X^{\prime}=V_{1}^{\prime} \backslash Y^{\prime}$.

Pela Proposição 4.1, temos que $\left(X^{\prime}, Y^{\prime}\right)$ não satisfaz $(r)$ em qualquer ponto de $0 \times \mathbb{R} \mathrm{em}$ $\mathbb{R}^{n} \times \mathbb{R}$ (por exemplo, considere a curva $q(s)=(p(s), s)$ de 0 em $X$ ). Mas, como $V_{1}^{\prime}$ é uma subvariedade de classe $C^{1}$, segue que $\left(X^{\prime}, Y^{\prime}\right)$ é $(b)$-regular.

Observação 4.6. O exemplo 4.3 descreve o primeiro exemplo de um par de estratos $(X, Y)$ satisfazendo $(b)$ mas não satisfazendo $(r)$, onde $X$ é a parte regular de uma variedade algébrica e $Y$ é o lugar singular. Compare isto com o caso de hipersuperfície complexa, onde (b)-regularidade, teste da razão e $(w)$-regularidade são equivalentes. Isto é uma conseqüência da equivalência da (b)-regularidade com a (c)-cosecance de Teissier, definida em [20] (referências para as implicações dando esta equivalência podem ser encontradas em [7]).

Exemplo 4.4. Seja $V_{2}=\left\{y^{4}=z^{4} x+x^{3}\right\} \subset \mathbb{R}^{3}$, e sejam $Y=$ eixo $z$ e $X$ o complementar de $Y$ em $V_{2}$. Considere $f_{2}(x, y, z)=-y^{4}+z^{4} x+x^{3}$, então para $m=(x, y, z) \in X,(x, y) \neq(0,0)$, temos que o espaço normal é gerado por:

$$
\nabla f_{2}(x, y, z)=\left(z^{4}+3 x^{2},-4 y^{3}, 4 z^{3} x\right)
$$

O vetor normal (direção) é:

$$
v(m)=\frac{\nabla f_{2}(x, y, z)}{\left\|\nabla f_{2}(x, y, z)\right\|}=\left(\frac{z^{4}+3 x^{2}}{\sqrt{C_{2}}}, \frac{-4 y^{3}}{\sqrt{C_{2}}}, \frac{4 z^{3} x}{\sqrt{C_{2}}}\right),
$$

onde $C_{2}=\left(z^{4}+3 x^{2}\right)^{2}+16 y^{6}+16 z^{6} x^{2}$.

Além disso, como $\tau \in T_{p} Y$ e $T_{p} Y$ é 1-dimensional, podemos assumir que $\tau=(0,0,1)$. Logo,

$$
\left|\pi_{N_{m} X}(\tau)\right|=|v(m) \cdot \tau|=\left|\frac{4 z^{3} x}{\sqrt{\left(z^{4}+3 x^{2}\right)^{2}+16 y^{6}+16 z^{6} x^{2}}}\right| .
$$

Elevando ao quadrado, temos que:

$$
\begin{aligned}
\left|\pi_{N_{m} X}(\tau)\right|^{2} & =\left|\frac{16 z^{6} x^{2}}{\left(z^{4}+3 x^{2}\right)^{2}+16 y^{6}+16 z^{6} x^{2}}\right| \\
& =\left|\frac{16 z^{6} x^{2}}{\left(z^{4}+3 x^{2}\right)^{2}+16\left(z^{4} x+x^{3}\right)^{\frac{3}{2}}+16 z^{6} x^{2}}\right| \\
& \leq\left|\frac{16 z^{6} x^{2}}{16\left(z^{4} x+x^{3}\right)^{\frac{3}{2}}}\right| .
\end{aligned}
$$

Elevando novamente ao quadrado, obtemos:

$$
\left|\pi_{N_{m} X}(\tau)\right|^{4}=\left|\frac{z^{12} x^{4}}{x^{3}\left(z^{4}+x^{2}\right)^{3}}\right|=\left|\frac{z^{12} x}{\left(z^{4}+x^{2}\right)^{3}}\right| \leq\left|\frac{z^{12} x}{z^{12}}\right|=|x| \longrightarrow 0 \text { quando } x \rightarrow 0 .
$$


Deste modo, segue-se que: $\lim _{m \rightarrow 0}\left|\pi_{N_{m} X}(\tau)\right|=0$. Portanto, $X$ é $(a)$-regular sobre $Y$.

Afirmanos que $X$ é $\left(b^{\prime}\right)$-regular sobre $Y$. De fato, seja $p=(0,0,0) \in Y$, então:

$$
\overrightarrow{m p}=\vec{m}=(x, y, z) \Longrightarrow \pi_{p}(\vec{m})=(0,0, z) \Longrightarrow \vec{m}-\pi_{p}(\vec{m})=(x, y, 0) .
$$

Logo, $\frac{\vec{m}-\pi_{p}(\vec{m})}{\left|\vec{m}-\pi_{p}(\vec{m})\right|}=\frac{(x, y, 0)}{\sqrt{x^{2}+y^{2}}}$.

Assim,

$$
\begin{aligned}
\left|\pi_{N_{m} X}\left(\frac{\vec{m}-\pi_{p}(\vec{m})}{\left|\vec{m}-\pi_{p}(\vec{m})\right|}\right)\right| & =\left|\left\langle v(m), \frac{(x, y, 0)}{\sqrt{x^{2}+y^{2}}}\right\rangle\right| \\
& =\left|\frac{z^{4} x+3 x^{3}-4 y^{4}}{\sqrt{\left(z^{4}+3 x^{2}\right)^{2}+16 y^{6}+16 z^{6} x^{2}} \sqrt{x^{2}+y^{2}}}\right| \\
& =\left|\frac{z^{4} x+3 x^{3}-4 z^{4} x-4 x^{3}}{\sqrt{\left(z^{4}+3 x^{2}\right)^{2}+16\left(z^{4} x+x^{3}\right)^{\frac{3}{2}}+16 z^{6} x^{2}} \sqrt{x^{2}+\left(z^{4} x+x^{3}\right)^{\frac{1}{2}}}}\right| \\
(\star) & =\left|\frac{3 z^{4} x+x^{3}}{\left[\left(z^{4}+3 x^{2}\right)^{2}+16\left(z^{4} x+x^{3}\right)^{\frac{3}{2}}+16 z^{6} x^{2}\right]^{\frac{1}{2}}\left[x^{2}+\left(z^{4} x+x^{3}\right)^{\frac{1}{2}}\right]^{\frac{1}{2}}}\right| .
\end{aligned}
$$

Afirmamos que $\lim _{m \rightarrow 0}(\star)=0$. De fato, pelo Lema de seleção da curva, existe uma curva analítica $\alpha(s)=\left(x_{0} s^{n}+\ldots, y_{0} s^{m}+\ldots, z_{0} s^{p}+\ldots\right)$, com $\alpha(0)=0$ e $\alpha(s) \in V_{2}$ se $s \neq 0$. Vamos verificar que $\lim _{s \rightarrow 0}(\star) \circ \alpha(s)=0$. Basta considerarmos os seguintes casos:

Caso I: $4 p+n>3 n \Leftrightarrow 4 p>2 n \Leftrightarrow 2 p>n$.

Caso II: $2 p=n$.

Caso III: $2 p<n$.

Deste modo, segue-se que: $\lim _{m \rightarrow 0}\left|\pi_{N_{m} X}\left(\frac{\vec{m}-\pi_{p}(\vec{m})}{\left|\vec{m}-\pi_{p}(\vec{m})\right|}\right)\right|=0$ e, consequentemente, temos que $X$ é $\left(b^{\prime}\right)$-regular sobre $Y$.

Portanto, $(b)$ vale para $(X, Y)$. Logo, pelo Teorema 4.2, deduzimos que $(X, Y)$ satisfaz $(r)$ em 0 , visto que $\operatorname{dim} Y=1$.

Agora, afirmamos que $(w)$ falha ao longo da curva $a(s)=\left(s^{4}, \sqrt[4]{2} \cdot s^{3}, s^{2}\right) \in V_{2}$. De fato, lembre-se que a direção normal a $X$ em $(x, y, z)$ é $\left(z^{4}+3 x^{2}:-4 y^{3}: 4 z^{3} x\right)$.

$\operatorname{Em} a(s)$ isto se torna:

$$
\left(s^{8}+3 s^{8}:-4.2^{\frac{3}{4}} s^{9}: 4 s^{6} s^{4}\right)=\left(4 s^{8}:-4.2^{\frac{3}{4}} s^{9}: 4 s^{10}\right)=\left(1:-2^{\frac{3}{4}} s: s^{2}\right) .
$$

Então, $d\left(T_{0} Y, T_{a(s)} X\right)=\left\langle\frac{\nabla f_{2}\left(s^{4}, \sqrt[4]{2} \cdot s^{3}, s^{2}\right)}{\left\|\nabla f_{2}\left(s^{4}, \sqrt[4]{2} \cdot s^{3}, s^{2}\right)\right\|},(0,0,1)\right\rangle=\frac{s^{2}}{\left(1+2^{\frac{3}{2}} s^{2}+s^{4}\right)^{\frac{1}{2}}} \sim 1$.

Além disso,

$$
\left|a(s)-\pi_{Y}(a(s))\right|=\left|\left(s^{4}, \sqrt[4]{2} \cdot s^{3}, s^{2}\right)-\left(0,0, s^{2}\right)\right|=\left|\left(s^{4}, \sqrt[4]{2} \cdot s^{3}, 0\right)\right|=\left|s^{3}(s, \sqrt[4]{2}, 0)\right| \sim s^{3} .
$$


Assim, $\frac{d\left(T_{0} Y, T_{a(s)} X\right)}{\left|a(s)-\pi_{Y}(a(s))\right|} \sim \frac{1}{s^{3}}$ que é ilimitado quando $s \rightarrow 0$.

Portanto, $(w)$ falha para $(X, Y)$ em 0.

Como no exemplo 4.3, podemos usar a Proposição 4.1 para mostrarmos que $(X \times \mathbb{R}, Y \times \mathbb{R})$ não satisfaz $(r)$ em $0 \times \mathbb{R}$ no $\mathbb{R}^{4}$, mas $(b)$ claramente vale.

Exemplo 4.5. Seja $V_{3}=\left\{y^{4}=z^{2} x^{5}+x^{7}\right\} \subset \mathbb{R}^{3}$, e sejam $Y=$ eixo $z$ e $X$ o complementar de $Y$ em $V_{3}$. Considere $f_{3}(x, y, z)=-y^{4}+z^{2} x^{5}+x^{7}$, então para $m=(x, y, z) \in X,(x, y) \neq(0,0)$, temos que o espaço normal é gerado por:

$$
\nabla f_{3}(x, y, z)=\left(5 z^{2} x^{4}+7 x^{6},-4 y^{3}, 2 z x^{5}\right)
$$

O vetor normal (direção) é:

$$
v(m)=\frac{\nabla f_{3}(x, y, z)}{\left\|\nabla f_{3}(x, y, z)\right\|}=\left(\frac{5 z^{2} x^{4}+7 x^{6}}{\sqrt{C_{3}}}, \frac{-4 y^{3}}{\sqrt{C_{3}}}, \frac{2 z x^{5}}{\sqrt{C_{3}}}\right)
$$

onde $C_{3}=\left(5 z^{2} x^{4}+7 x^{6}\right)^{2}+16 y^{6}+4 z^{2} x^{10}$.

Além disso, se $\tau \in T_{0} Y$, podemos assumir que $\tau=(0,0,1)$, visto que $T_{0} Y$ é 1-dimensional. Logo,

$$
\left|\pi_{N_{m} X}(\tau)\right|=|v(m) \cdot \tau|=\left|\frac{2 z x^{5}}{\sqrt{\left(5 z^{2} x^{4}+7 x^{6}\right)^{2}+16 y^{6}+4 z^{2} x^{10}}}\right| .
$$

Elevando ambos os membros ao quadrado, temos que:

$$
\begin{aligned}
\left|\pi_{N_{m} X}(\tau)\right|^{2} & =\left|\frac{4 z^{2} x^{10}}{\left(5 z^{2} x^{4}+7 x^{6}\right)^{2}+16 y^{6}+4 z^{2} x^{10}}\right| \\
& =\left|\frac{4 z^{2} x^{10}}{\left(5 z^{2} x^{4}+7 x^{6}\right)^{2}+16\left(z^{2} x^{5}+x^{7}\right)^{\frac{3}{2}}+4 z^{2} x^{10}}\right| \\
& \leq\left|\frac{4 z^{2} x^{10}}{16\left(z^{2} x^{5}+x^{7}\right)^{\frac{3}{2}}}\right| .
\end{aligned}
$$

Elevando novamente ao quadrado, obtemos:

$$
\left|\pi_{N_{m} X}(\tau)\right|^{4}=\left|\frac{z^{4} x^{20}}{4 x^{15}\left(z^{2}+x^{2}\right)^{3}}\right|=\left|\frac{z^{4} x^{5}}{4\left(z^{2}+x^{2}\right)^{3}}\right| .
$$

Vamos dividir em 2 casos:

Caso I: $p=\left(0,0, z^{\prime}\right)$, com $z^{\prime} \neq 0$. Aqui, é claro que $\lim _{m \rightarrow p}\left|\frac{z^{4} x^{5}}{4\left(z^{2}+x^{2}\right)^{3}}\right|=0$. 
Caso II: $p=(0,0,0)$. Aqui, como no exemplo anterior, podemos encontrar uma curva analítica $\alpha(s)=\left(x_{0} s^{n}+\ldots, y_{0} s^{m}+\ldots, z_{0} s^{p}+\ldots\right)$, com $\alpha(0)=0$ e $\alpha(s) \in V_{3}$ se $s \neq 0$, tal que:

$$
\lim _{m \rightarrow p}\left|\frac{z_{0}^{4} x_{0}^{5} s^{4 p+5 n}+\ldots}{4\left(z_{0}^{2} s^{2 p}+x_{0}^{2} s^{2 n}\right)^{3}+\ldots}\right|=0
$$

e consequentemente,

$$
\lim _{m \rightarrow p}\left|\frac{z^{4} x^{5}}{4\left(z^{2}+x^{2}\right)^{3}}\right|=0
$$

Deste modo, segue-se que: $\lim _{m \rightarrow 0}\left|\pi_{N_{m} X}(\tau)\right|=0$. Portanto, $X$ é $(a)$-regular sobre $Y$.

Agora, considere $p=\left(0,0, z_{0}\right) \in Y$, então:

$$
\overrightarrow{m p}=\left(x, y, z-z_{0}\right) \Longrightarrow \pi_{p}(\overrightarrow{m p})=\left(0,0, z-z_{0}\right) \Longrightarrow \overrightarrow{m p}-\pi_{p}(\overrightarrow{m p})=(x, y, 0)
$$

Consequentemente, $\frac{\overrightarrow{m p}-\pi_{p}(\overrightarrow{m p})}{\left|\overrightarrow{m p}-\pi_{p}(\overrightarrow{m p})\right|}=\frac{(x, y, 0)}{|(x, y, 0)|}=\frac{(x, y, 0)}{\sqrt{x^{2}+y^{2}}}$.

Assim,

$$
\begin{aligned}
\left|\pi_{N_{m} X}\left(\frac{\overrightarrow{m p}-\pi_{p}(\overrightarrow{m p})}{\left|\overrightarrow{m p}-\pi_{p}(\overrightarrow{m p})\right|}\right)\right| & =\left|\left\langle v(m), \frac{(x, y, 0)}{\sqrt{x^{2}+y^{2}}}\right\rangle\right| \\
& =\left|\frac{5 z^{2} x^{5}+7 x^{7}-4 y^{4}}{\sqrt{\left(5 z^{2} x^{4}+7 x^{6}\right)^{2}+16 y^{6}+4 z^{2} x^{10}} \sqrt{x^{2}+y^{2}}}\right| .
\end{aligned}
$$

Elevando ambos os membros ao quadrado, obtemos:

$$
\begin{aligned}
\left|\pi_{N_{m} X}\left(\frac{\overrightarrow{m p}-\pi_{p}(\overrightarrow{m p})}{\left|\overrightarrow{m p}-\pi_{p}(\overrightarrow{m p})\right|}\right)\right|^{2} & =\left|\frac{\left(5 z^{2} x^{5}+7 x^{7}-4 y^{4}\right)^{2}}{\left[\left(5 z^{2} x^{4}+7 x^{6}\right)^{2}+16 y^{6}+4 z^{2} x^{10}\right]\left(x^{2}+y^{2}\right)}\right| \\
& =\left|\frac{\left[5 z^{2} x^{5}+7 x^{7}-4\left(z^{2} x^{5}+x^{7}\right)\right]^{2}}{\left[\left(5 z^{2} x^{4}+7 x^{6}\right)^{2}+16\left(z^{2} x^{5}+x^{7}\right)^{\frac{3}{2}}+4 z^{2} x^{10}\right]\left(x^{2}+y^{2}\right)}\right| \\
& =\left|\frac{x^{10}\left(z^{2}+6 x^{2}\right)^{2}}{\left(x^{2}+y^{2}\right)\left[\left(5 z^{2} x^{4}+7 x^{6}\right)^{2}+16\left(z^{2} x^{5}+x^{7}\right)^{\frac{3}{2}}+4 z^{2} x^{10}\right]}\right| \\
& \leq\left|\frac{x^{8}\left(z^{2}+6 x^{2}\right)^{2}}{\left(5 z^{2} x^{4}+7 x^{6}\right)^{2}+16\left(z^{2} x^{5}+x^{7}\right)^{\frac{3}{2}}+4 z^{2} x^{10}}\right| \\
& \leq\left|\frac{x^{8}\left(z^{2}+6 x^{2}\right)^{2}}{16\left(z^{2} x^{5}+x^{7}\right)^{\frac{3}{2}}}\right| .
\end{aligned}
$$

Elevando novamente ao quadrado, temos que:

$$
\left|\pi_{N_{m} X}\left(\frac{\overrightarrow{m p}-\pi_{p}(\overrightarrow{m p})}{\left|\overrightarrow{m p}-\pi_{p}(\overrightarrow{m p})\right|}\right)\right|^{4}=\left|\frac{x^{16}\left(z^{2}+6 x^{2}\right)^{4}}{16 x^{15}\left(z^{2}+x^{2}\right)^{3}}\right|=\left|\frac{x\left(z^{2}+6 x^{2}\right)^{4}}{16\left(z^{2}+x^{2}\right)^{3}}\right| \longrightarrow 0 \text { quando } x \rightarrow 0 \text {. }
$$

Então, $\lim _{m \rightarrow p}\left|\pi_{N_{m} X}\left(\frac{\overrightarrow{m p}-\pi_{p}(\overrightarrow{m p})}{\left|\overrightarrow{m p}-\pi_{p}(\overrightarrow{m p})\right|}\right)\right|=0$, ou seja, $X$ é $\left(b^{\prime}\right)$-regular sobre $Y$. 
Portanto, (b) vale para o par de estratos $(X, Y)$. Logo, pelo Teorema 4.2, deduzimos que $(X, Y)$ satisfaz $(r)$ em 0 , visto que $\operatorname{dim} Y=1$.

Note que $(w)$ falha ao longo da curva $a(s)=\left(s^{4}, \sqrt[4]{2} \cdot s^{7}, s^{4}\right) \in V_{3}$. E, como no exemplo 4.3, podemos aplicar a Proposição 4.1 para mostrarmos que $(X \times \mathbb{R}, Y \times \mathbb{R})$ não satisfaz $(r)$ em $0 \times \mathbb{R}$ no $\mathbb{R}^{4}$, mas (b) claramente vale.

Observação 4.7. Os exemplos 4.3 e 4.4 mostram que $(r)$ e $(w)$ não são invariantes sobre $C^{1}$-difeomorfismos. Portanto $(b)$ é mais natural do ponto de vista da topologia diferencial, pois ela é $C^{1}$-invariante.

\subsection{Campos de vetores rugosos}

Em [24], Verdier mostra que existem campos de vetores rugosos sobre uma estratificação $(w)$-regular e deriva trivialização rugosa. Entretanto, pode ser impossível estender um campo de vetor constante sobre um estrato base $Y$, para um campo de vetor rugoso sobre um estrato adjacente $X$, quando $(X, Y)$ é (b)-regular. Esta é uma consequência da próxima Proposição e a existência de exemplos $(b)$-regulares que não satisfazem $(w)$.

Definição 4.3. Sejam A um conjunto subanalítico de um espaço vetorial euclidiano $W, S$ uma estratificação de Whitney de $A$ e $\nu: A \longrightarrow \mathbb{R}$ um campo de vetor. Dizemos que $\nu$ é um campo de vetor rugoso, se para qualquer estrato $X_{i},\left.f\right|_{X_{i}}$ é de classe $C^{\infty}$ e, se para qualquer $m \in S$, existe uma vizinhança $U$ de $m$ e uma constante $c>0$ tal que

$$
\left|\nu\left(m^{\prime}\right)-\nu(p)\right| \leq c\left|m^{\prime}-p\right| \text {, para todo } m^{\prime} \in U \cap X_{i} \text { e todo } p \in U \cap A .
$$

Proposição 4.2. Sejam $X$ uma subvariedade de classe $C^{2}$ no $\mathbb{R}^{m}$ e $Y=\mathbb{R}^{n} \times\{0\} \subset \mathbb{R}^{m}$. Suponha que cada campo de vetor constante $\left\{\frac{\partial}{\partial p_{i}}\right\}, i=1, \ldots, n$ sobre $Y$, se estende à um campo de vetor rugoso em $X \cup Y$. Então, $X$ é $(w)$-regular sobre $Y$.

Demonstração: Seja $\widetilde{v_{i}}$ a extensão de $\frac{\partial}{\partial p_{i}}$.

Note que, para cada $i$ existe uma constante $c>0$ e uma vizinhança $U$ de zero, tal que

$$
\left|\widetilde{v_{i}}(m)-\frac{\partial}{\partial p_{i}}\right| \leq c|m-p|, \text { para todo } m \in U \cap X \text { e todo } p \in U \cap Y .
$$

Podemos assumir que $c$ e $U$ são os mesmos para todo $i$.

Seja $m \in U$. Então,

$$
d\left(\frac{\partial}{\partial p_{i}}, T_{m} X\right) \leq\left|\frac{\partial}{\partial p_{i}}-\widetilde{v_{i}}(m)\right| \leq c|m-p| .
$$

Logo, $d\left(\frac{\partial}{\partial p_{i}}, T_{m} X\right) \leq c|m-p|$, para todo $m \in U \cap X$ e todo $p \in U \cap Y$. (*)

Tome $v \in T_{p} Y$ com $|v|=1$, então: $v=\sum_{i=1}^{n} a_{i} \frac{\partial}{\partial p_{i}}$, com $\sum_{i=1}^{n} a_{i}^{2}=1$. 
Sejam $N_{m} X$ o complemento ortogonal de $T_{m} X$ em $\mathbb{R}^{m}$ e $\pi_{m}: \mathbb{R}^{m} \longrightarrow N_{m} X$ a projeção ortogonal.

Assim, $d\left(v, T_{m} X\right)=\left|\pi_{m}(v)\right|$

$$
\begin{aligned}
& =\left|\sum_{i=1}^{n} a_{i} \pi_{m}\left(\frac{\partial}{\partial p_{i}}\right)\right| \\
& \leq \sum_{i=1}^{n}\left|\pi_{m}\left(\frac{\partial}{\partial p_{i}}\right)\right| \\
& =\sum_{i=1}^{n} d\left(\frac{\partial}{\partial p_{i}}, T_{m} X\right) \\
& \leq n . c|m-p|, \text { por }(*) .
\end{aligned}
$$

Logo, $d\left(T_{p} Y, T_{m} X\right)=\sup _{|v|=1} d\left(v, T_{m} X\right) \leq n . c|m-p|$, para todo $m \in U \cap X$ e todo $p \in U \cap Y$.

Daí, $X$ é $(w)$-regular sobre $Y$ em 0 .

Repetindo o argumento acima para cada $p \in Y$, obtemos que $X$ é $(w)$-regular sobre $Y$.

Corolário 4.4.1. Sejam $A=X \cap B$ um subconjunto fechado do $\mathbb{R}^{m}, B \cup X=\emptyset, X$ uma subvariedade de classe $C^{2}$ e $B$ um subconjunto fechado.

Seja $(B, S)$ uma estratificação $(w)$-regular, com cada estrato uma subvariedade de classe $C^{2}$. Então, a estratificação $S^{\prime}$ de A, obtida adicionando $X$ à $S$, é $(w)$-regular se, e somente se, todo campo de vetor rugoso sobre $B$ tangente à $S$ pode ser extendido para um campo de vetor rugoso sobre A tangente à $S^{\prime}$.

Demonstração: $(\Rightarrow)$ Segue da Proposição 4.2.

$(\Leftarrow)$ Está provado por Verdier em [24]. 


\section{Referências Bibliográficas}

[1] K. Bekka, C-régularité et trivialité topologique, Singularity theory and its applications, Part I (Coventry, 1988/1989), Lecture Notes in Math. 1462, Springer, Berlin, 1991, 42-62.

[2] K. Bekka, Regular Stratification of subanalytics Sets, Bull London Math. Soc. 25, 1993, 7-16.

[3] K. Bekka and D. Trotman, Weakly Whitney stratified sets, Real and Complex singularities (São Carlos 1998) J. W. Bruce and F. Tari, Chapman \& Hall/CRC Res. Notes Math. 412, 2000, 1-15.

[4] E. Bierstone and P. Milman, Semianalytic and subanalytic sets, Publ. IHES 67, 1988, 5-42.

[5] E. Bierstone and P. Milman, Subanalytic Geometry, Model theory, algebrar and geometry, Math. Sci. Res. Inst. Publ. 39, Cambridge, Univ. Press, 2000, 151-172.

[6] J. Briançon and J. P. Speder, La trivialité topologique n'implique pas les conditions de Whitney, C. R. Acad. Sci. Paris Sér. A 280, 1975, 365-367.

[7] J. Briançon and J. P. Speder, Les conditions de Whitney impliquent $\mu^{*}$-constant, Ann. Inst. Fourier (Grenoble), 26 (2), 1976, 153-163.

[8] H. Brodersen and D. Trotman, Whitney (b)-regularity is weaker than Kuo's Ratio Test for Real algebraic stratifications, Math. Scand, Vol. 45, 1979, 27-34.

[9] Z. Denkowska, K. Wachta, J. Stasica, Stratification des ensembles sous-analytiques avec les propriétés (A) et (B) de Whitney, Univ. Iagel Acta Math. 25, 1985, 183-188.

[10] A. Hamm and D. T. Lê, Un théorème de Zariski du type de Lefschetz, Ann. Sci. École Norm. Sup. (4) 6, 1973, 317-355.

[11] T. C. Kuo, The ratio test for Whitney stratifications, Proceedings of Liverpool Singularities Symposium I., Lecture Notes in Math. 192, Springer-Verlag, 1971, 141-149.

[12] S. Lojasiewicz, Ensembles semi-analytiques, IHES Mathematics, 1965.

[13] Yung-Chen Lu, Singularity Theory and an Introduction to Catastrophe Theory, SpringerVerlag, New York Heidelberg Berlin, Capítulo 5, 1938, 121-141. 
[14] J. P. Markus, Analytic and Geometry Study of Stratified Spaces, Springer, 2001, 15-41.

[15] J. N. Mather, Notes on topological stability,Lecture Notes, Harvard University, 1970.

[16] J. N. Mather, Stratification and Mappings, Dynamical Systems (Proc. Sympos. Univ. Bahia, Salvador, 1971), Academic Press, New York, 1973, 195-232.

[17] J. W. Milnor, Singular points of complex hypersurfaces, Annals of Mathematics Studies, Princeton University Press, 61, 1968.

[18] J. W. Milnor, Morse Teory, Annals of Mathematics Studies, Princeton University Press, 51, 1963.

[19] A. T. A. do Nascimento, Distância Geodésica em Conjuntos Subanalíticos, Dissertação de Mestrado, Fortaleza, 2003, 5-22.

[20] B. Teissier, Variétés polaires I. Invariants polaires des singularités d'hypersurfaces, Invent, Math. 40, 1977, 267-292.

[21] R. Thom, Ensembles et morphisms stratifiés, Bulletin AMS 75, 1969, 240-284.

[22] D. Trotman, Counterexamples in stratification theory: two discordant horns in Real and complex singularities, (Proceedings of the Nordic Summer School/NAVF Symposium Math., Olso, 1976), Sijthoff and Noordhoff, Alphen aan de Rijn, 1977, 679-686.

[23] D. Trotman, Stratifications of analytic spaces, School and Workshop on the Geometry and Topology of Singularities and a celebration of the first 60 years of Lê Dũng Tráng, Cuarnavaca, Mexico, 8-20 January, 2007.

[24] J.- L. Verdier, Stratifications de Whitney et Théorème de Bertini - Sard, Invent. Math. 36, 1976, 295-312.

[25] C. T. C. Wall, Regular stratifications, Dynamical Systems, Warwick 1974, Lecture Notes in Math. 468, (Springer, Berlin, 1975), 332-344.

[26] H. Whitney, Elementary structure of real algebraic varieties, Annals of Mathematics 66, 1957, 545-556.

[27] H. Whitney, Local properties of analytic varieties, Differential and Combinatorial Topology, Princeton, Univ. Press, 1965, 205-244.

[28] H. Whitney, Tangents to an analytic variety, Annals of Mathematics 81, 1965, 496-549. 